\title{
A New Catalogue of Galactic Novae: Investigation of the MMRD relation \& Spatial Distribution
}

\author{
Aykut Özdönmez ${ }^{1}$, Ergün Ege ${ }^{1,2}$, Tolga Güver ${ }^{2,3}$, and Tansel Ak ${ }^{3}$ \\ ${ }^{1}$ Istanbul University, Graduate School of Science and Engineering, 34116, Beyazut, Istanbul, Turkey \\ ${ }^{2}$ Istanbul University Observatory Research and Application Center, Beyazlt, 34119 Istanbul, Turkey \\ ${ }^{3}$ Istanbul University, Faculty of Science, Department of Astronomy and Space Sciences, Beyazit, 34119, Istanbul, Turkey
}

Accepted 2018 February 13. Received 2018 February 13; in original form 2017 November 06.

\begin{abstract}
In this study, a new Galactic novae catalogue is introduced collecting important parameters of these sources such as their light curve parameters, classifications, full width half maximum (FWHM) of $\mathrm{H}_{\alpha}$ line, distances and interstellar reddening estimates. The catalogue is also published on a website with a search option via a SQL query and an online tool to re-calculate the distance/reddening of a nova from the derived reddening-distance relations. Using the novae in the catalogue, the existence of a maximum magnitude-rate of decline (MMRD) relation in the Galaxy is investigated. Although an MMRD relation was obtained, a significant scattering in the resulting MMRD distribution still exists. We suggest that the MMRD relation likely depends on other parameters in addition to the decline time, as $\mathrm{FWHM} \mathrm{H}_{\alpha}$, the light curve shapes. Using two different samples depending on the distances in the catalogue and from the derived MMRD relation, the spatial distributions of Galactic novae as a function of their spectral and speed classes were studied. The investigation on the Galactic model parameters implies that best estimates for the local outburst density are 3.6 and $4.2 \times 10^{-10} \mathrm{pc}^{-3} \mathrm{yr}^{-1}$ with a scale height of 148 and $175 \mathrm{pc}$, while the space density changes in the range of $0.4-16 \times 10^{-6}$ $\mathrm{pc}^{-3}$. The local outburst density and scale height obtained in this study infer that the disk nova rate in the Galaxy is in the range of $\sim 20$ to $\sim 100 \mathrm{yr}^{-1}$ with an average estimate $67_{-17}^{+21} \mathrm{yr}^{-1}$.
\end{abstract}

Key words: Cataclysmic Variables: Novae, distances

\section{INTRODUCTION}

Novae are defined as systems with the largest outburst amplitude among other sub-types of cataclysmic variables, which is a shortperiod interacting binary system containing a white dwarf (WD) and a donor star (Warner 1995). A nova outburst occurs on the surface of a white dwarf where material accumulates from a donor star until the pressure and temperature are high enough to trigger a thermonuclear runaway that ejects the accreted envelope (Bode \& Evans 2008; Woudt \& Ribeiro 2014). Systems with much smaller recurrence times, called recurrent novae (RNe), show at least two outbursts in the observational history, while systems classified as classical novae $(\mathrm{CN})$ have only one outburst discovered. The relatively massive WDs and higher mass transfer rates in RNe probably shorten the recurrence time (Starrfield et al. 1985; Yaron et al. 2005). Traditionally, the classification of novae is based on outburst properties. They are classified by their speed class (Gaposchkin 1957), determined via their decline times, as fast or slow novae, by their spectral class (Williams 1992), determined via the form of the early outburst spectra, as Fe II, He/N or hybrid novae. Strope et al. (2010) clas-

^ E-mail: aykut.ozdonmez@ogr.iu.edu.tr sified Galactic novae based on the light curve shapes into smooth (S), plateau (P), dust dip (D), cusp (C), oscillations (O), flat topped $(\mathrm{F})$, and jitter $(\mathrm{J})$. Besides, the classifications based on the outburst properties, these systems can also be classified via the evolutionary state of the donor star (Darnley et al. 2012) into main sequence, sub-giant or red giant star novae; or via the population of the system in the host galaxy (Della Valle \& Livio 1998) into disk or bulge novae.

Novae are important sources to study for a number of reasons. For example, novae, particularly, RNe that host a high-mass WD (likely close to the Chandrasekhar limit) with sub-giant or red giant donor star, are considered as potential progenitors of type Ia supernovae, which are used to measure the accelerating expansion of the Universe (Maoz et al. 2014). They are also important sources for the enrichment of the interstellar medium in carbon, nitrogen, oxygen and lithium (Starrfield et al. 2009; Tajitsu et al. 2015). They are X-ray and high energy gamma-ray sources (Chomiuk et al. 2014). Moreover, nova eruptions also allow studies of the commonenvelope process and of the shock physics (Chomiuk et al. 2014).

With the advances in recorded nova outbursts and obtained data over the last decades, the number of novae with known fundamental parameters increased, and their outburst mechanism, nature 
of light curves and spectra became more understandable. For example, Strope et al. (2010) represented well-determined light curves including peak magnitudes and dates, decline times, the quiescent magnitudes, and their light curve classes. To quantify RN candidates which have been classified as CN, Pagnotta \& Schaefer (2014) analyzed the Galactic nova sample based on the information on the light curves and spectra, and recognized $\mathrm{RN}$ candidates since they have smaller outburst amplitudes, larger orbital periods, infrared colors indicating the existence of giant donor stars, high expansion velocities from full width half maximum (FWHM) of $\mathrm{H}_{\alpha}$ line at $6563 \AA$, high excitation lines such as Fe x or He II near outburst peak, a light curve shape class of P-type, and WD masses greater than $1.2 \mathrm{M}_{\odot}$. From the new observations, the number of discovered bulge Galactic novae were also increased (Mróz et al. 2015), and the nova rate was calculated both for disk and bulge systems (Shafter 2017; Mróz et al. 2015).

Recently, we determined the distances of a large sample of novae using a systematic approach (Özdönmez et al. 2016), which depends on the reddening-distance relation derived by utilizing the unique location of red clump stars on colour-magnitude diagrams from 2MASS (Skrutskie et al. 2006), UKIDSS (Lucas et al. 2008)) and VISTA VVV (Saito et al. 2012) near-infrared surveys. The results are consistent with the distances inferred from parallax method within the stated errors, and the method has the advantage of being applicable to a large number of novae. Hence, the existence of a maximum magnitude - rate of decline (MMRD) relation for the Galaxy, which has long been questioned and has been used to calculate absolute magnitudes of novae, can be investigated with these new distances and light curve parameters.

There have been numerous characterizations of the MMRD relation for Galactic (e.g. Cohen 1985; Downes \& Duerbeck 2000) and extragalactic nova populations (e.g. Capaccioli et al. 1990; della Valle \& Livio 1995; Darnley et al. 2006). A recent study by Kasliwal et al. (2011) and Shara et al. (2017) has revealed a number of apparently faint, yet relatively rapidly fading novae in M31 and M87, which caused the authors to question whether an MMRD relation is justified at all. Theoretically, the WD mass determines the main trend of the MMRD relation, and the ignition (initial envelope) mass, in other words, the mass-accretion rate to the WD causes the scatter in the MMRD relation (Yaron et al. 2005; Hachisu \& Kato 2010). If the mass-accretion rate to the WD is relatively larger, the ignition mass is smaller, hence the peak brightness is fainter. However, the heterogeneity in nova light curves suggests that a single parameter may not characterize the decline well. Thus, other properties such as spectroscopic behavior, light curve shape which characterize novae in different ways may resolve the scatter in the MMRD relation.

In this study, we compile a new Galactic nova catalogue, which provide information on the nova population as described in Section 2. Using the data in the catalogue, we investigate absolute magnitude-decline time distributions to obtain the MMRD relation of the Galactic novae in Section 3. The statistical analyses of the Galactic novae on the decline times, absolute magnitudes at maximum outburst, and spatial distributions are given in 4 . We also investigate Galactic model parameters as space density and scale height in the same section. Using the obtained Galactic model parameters, we also calculated the nova rate of disk novae. Finally, Section 6 represents a review of the results with a discussion of the possible uncertainties.

\section{THE CATALOGUE FOR THE GALACTIC NOVAE}

We compile a new catalogue of Galactic novae for which the distance information is acquirable. The catalogue contains 291 novae and almost all their important parameters such as their light curve parameters (amplitude A, maximum magnitude at outburst $V_{\text {max }}$, decline times $t_{2}, t_{3}$ ), shape of the light curve, spectral, recurrence classifications, FWHM $\mathrm{H}_{\alpha}$ and orbital period $P_{\text {orb }}$. The distances and/or interstellar reddening estimates are crucial parameters to obtain the luminosity function, spatial distributions or absolute physical parameters of these binary systems. In the catalogue, we included all the distance information of more than 150 novae in the literature obtained from the expansion/trigonometric parallaxes or from reddening-distance relations (RDRs; Özdönmez et al. 2016). The distances for the remaining novae could be obtained from MMRD relations. Besides, all reddening estimations for these nova systems were collected from the literature together with their measurement methods.

The distance measurements of the novae in the catalogue depends on two approaches; parallax and RDR methods. The most reliable method is the trigonometric parallax, but the number of novae with trigonometric parallax is less than 10 in the entire observational history even with GAIA DR1 (Gaia Collaboration et al. 2016). Distances can also be calculated by following the expansion parallax method which depends on the angular expansion of the resolved nova shell. However, it is not free from systematic biases due to the complex structure of shells, non-uniform shell expansion, inconstant expansion velocities etc. In the catalogue, $~ 30$ novae have expansion parallaxes. For some novae, e.g. LW Ser, QV Vul, LV Vul, the distances from expansion parallaxes, determined at different phases of the outburst and/or from the profiles of various spectral lines, show significant scatter. In the previous study (Özdönmez et al. 2016), we utilized a new distance calculation method for these systems via RDRs that helped us to determine the distances for nearly all known Galactic novae at low Galactic latitudes. In this RDR method, the uncertainties of the distance measurements arise from reddening estimates of novae as well as the shallow increase in the RDRs in some directions of the Galaxy. The comparison of the distances calculated following this method with that from the parallax method in Özdönmez et al. (2016) showed that two methods are in good agreement within the stated errors, except for LV Vul, LW Ser, and QV Vul.

There are a number of studies that compile data from AAVSO ${ }^{1}$ (American Association of Variable Star Observers) and/or from literature to present the light curve properties of Galactic novae, e.g. Warner (1987); Shafter (1997); Burlak \& Henden (2008), but a large number of qualified light curves with extensive time coverage (often until quiescence) that go deep and have many observations each day are only presented by Strope et al. (2010). As mentioned in Strope et al. (2010), the compilations, which include observations before the AAVSO data started, missed the peak of almost half of the novae, and correspondingly the decline times $\left(t_{2}, t_{3}\right)$ are not well determined. Besides, adopting a maximum brightness $\left(V_{\max }\right)$ and decline times during nova outburst without comprehensive knowledge of the light curve shape of a nova may be erroneous. Hence, the light curve shapes of novae in our sample were additionally checked using the AAVSO archive and/or related studies of the nova in question. If the novae have enough observations to clearly represent declining in the light curve, we assumed that the light curve parameters of the novae are adequately reliable. Recently, Pagnotta \& Schaefer

\footnotetext{
1 https://www.aavso.org/
} 
(2014) analyzed the Galactic nova sample from the information on the light curves and spectra to quantify RN candidates. FWHM $\mathrm{H}_{\alpha}$ around the outburst maximum is one of the important parameters along with the amplitude of the outburst, decline times, light curve shapes and excitation lines that correspond to the recurrence of a novae (Pagnotta \& Schaefer 2014). The expansion velocity of the ejected material in a nova outburst is measured using widths of emission lines, generally using the $\mathrm{H}_{\alpha}$ line. It may also help to clarify the scatter in the MMRD relation as discussed in section 3.

Our catalogue is published on the website ${ }^{2}$. For 189 Galactic novae, the RDRs were derived from the method described in Özdönmez et al. (2016), and these relations are shown at individual webpages. It also contains an algorithm to reproduce calculations of either the distance or the reddening for a given value, from derived RDRs. The website contains a main table in which the adopted parameters are given and an SQL based query can be performed to search novae systems. All references and detailed descriptions for the parameters are given on the website as well. The catalogue will be updated frequently with most recent determined parameters and new RDRs for the novae, which were not investigated.

We listed the novae in the catalogue in two tables. The novae with distances calculated from parallaxes or RDRs are presented in Table 5. The novae used to derive the MMRD relation are given in Table 5a. Unreliable or questionable parameters for the novae, which are not used in the derivation of the MMRD relation, are listed in Table $5 \mathrm{~b}$. The remaining novae that do not have any distance measurement are listed in Table 6. The light curve parameters of the novae in Table 6a are well-determined to allow the accurate measurements from the MMRD relation.

\section{THE MMRD RELATION OF GALACTIC NOVAE}

In this section, we aim to analyze the existence of the MMRD relation for the Galactic novae that has long been questioned. In order to analyze MMRD relations, we used only well-determined light curve parameters and distances as given in Table 5a. Some of the novae (e.g. CK Vul, CT Ser, V2674 Oph) do not have enough observations to obtain a reliable maximum magnitude and/or decline rates from outburst light-curves, and are not included in the analyses. Such novae are given in Table $5 \mathrm{~b}$. We also give information on the reason of why a nova in Table $5 \mathrm{~b}$ is not used for the investigation of the MMRD relation. For example, the distances of the novae BY Cir, V1301 Aql and V1493 Aql obtained from RDRs vary largely owing to different extinction measurement methods. Even a small variation in the reddening estimate changes the distance calculation drastically, for instance in case of V394 CrA, Q Cyg, V373 Sct, V4160 Sgr, V5116 Sgr. For some other novae (e.g. V394 CrA, V368 Sct), more reliable reddening measurements are required to calculate accurate distances using this method. Since the distance calculation is strongly correlated with the reddening estimate, we also mark the novae where the distance calculation depends on only one reddening estimate.

Using the Galactic nova samples in which the distance measurements depends on two different approaches (parallax and/or RDR), we plot $M_{V, \max }-t_{2,3}$ distributions (see, Fig. 1) to search for MMRD relations. For the main sample, we preferred to use the distances from the parallax method switching to that from RDRs.

2 http://highenergyastro.istanbul.edu.tr/novae_cat
The other sample contains the novae whose distances are calculated using a particular distance measurement method. Hence, we test systematic differences between MMRD relations obtained from different distance measurement methods. On the other hand, a number of studies that are based on theoretical models, suggest that the classical MMRD relation does not work properly for RNe, as seen in Fig. 2. For these reasons, we analyzed the distributions by separating the RNe and RN candidates from classical novae. Note that while obtaining MMRD relations, we excluded only strong RN candidates given in Pagnotta \& Schaefer (2014), for which many of the indicators strongly point to them being recurrent but only one outburst has been detected.

The uncertainty in the absolute magnitude at outburst maximum was estimated by accounting for the uncertainty of the distances and extinction, but for the calculation of the total uncertainty, the uncertainty in the maximum magnitude should also be taken into account. However, the brightest observed V-band magnitudes are usually adopted for the maximum magnitude during the outburst, and it may lead to an erroneous estimate in case the nova was detected after already passing the true maximum. This observational effect may lead to a larger uncertainty than the typical instrumental errors on maximum magnitudes, and therefore the uncertainty of the maximum magnitudes has usually not been considered in the MMRD studies. Similar problems exist in defining the decline times that are usually obtained by binning or interpolating the light curves for a given maximum magnitude. There can be multiple measurements of decline times for a light curve that fluctuates around the given magnitude. Thus, we only considered the novae with well-observed light-curves, and we adopted $\pm 10 \%$ of the maximum magnitude value as the error to represent systematic uncertainties. Moreover, we investigated $M_{V, \max }-t_{2,3}$ distributions by considering the classification of light curve shapes to analyze the dependence on MMRD relation due to the non-linear decline of novae possibly arising from jitters, cusps, and dips in light curve, at the end of this section.

We obtained MMRD relations for $t_{2}$ and $t_{3}$ decline times with the use of three different novae samples according to the considered distance measurement methods. The MMRD relations appear almost linear; $M_{V, \max }=a+b \times \log t_{2,3}$. The coefficients of the fit and the result from regression analyses by taking into account uncertainties in only the y-axis are given in Table 1 . The coefficients of the MMRD relations obtained from the three different samples of novae are within the stated errors, but the relations obtained from the parallax+RDR sample for both $t_{2}$ and $t_{3}$ times have smaller slopes (coefficient b) and larger y-intercepts (coefficient a) than that from the other samples. Even though the novae in the RDR sample have larger errors and show a larger scattering in absolute magnitude at maximum, the coefficients are similar to those of the other relations. Thus, we assume that there is no systematic bias between the derived relations when different distance measurement approaches are used. The reduced $\chi^{2}$ is expected to be less than 1 for considering model to be over-fitting data, but the the $\chi_{\text {red }}^{2}$ of the MMRD relations obtained in this study are greater than 2 . In addition, the $\pm 1 \sigma$ is about $1 \mathrm{mag}$, while greatest deviations from MMRD relations are about 2 mag. Nevertheless, a linear equation appears to be an adequately (but not fully) fit to the data. We used the MMRD relations from the parallax+RDR sample in the further investigations, since the relations were obtained from a larger number of novae, and have smaller standard errors and $\chi_{\text {red }}^{2}$. Our results are similar to the most popular MMRD relation of Downes \& Duerbeck (2000), in which the $1 \sigma$ scatter was \pm 0.6 mag with a deviation as large as 1.6 mag.

The previous studies on the MMRD relation show a common 

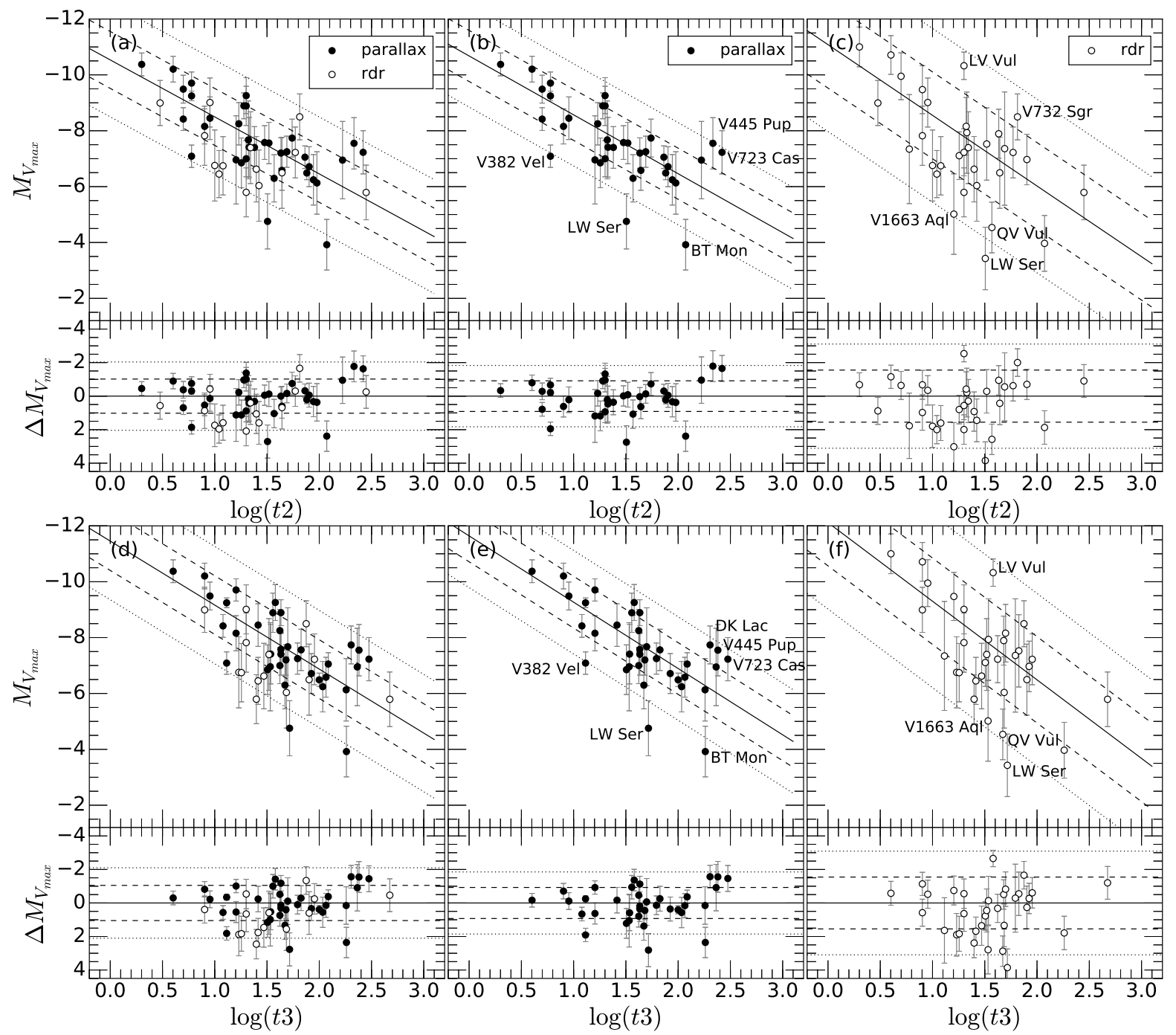

Figure 1. $M_{V, \text { max }}-t_{2,3}$ distributions of Galactic novae: (a,b,c) and (d,e,f) for $t_{2}$ and $t_{3}$, respectively. (a, d) for the novae with distances obtained from either parallax or RDR , (b, e) only with parallax measurements and (c, f) only with distance measurements from RDR. In the upper panels, the black lines represent the MMRD relation, the dashed and dotted lines show an uncertainty of $\pm 1 \sigma$ and $\pm 2 \sigma$ of the fit, respectively. The residuals are shown in the lower panels. The names of novae with relatively larger scattering are given.

Table 1. The results of regression analyses for deriving MMRD relations according to $t_{2}$ and $t_{3}$ times. Here, $\mathrm{N}$ is number of novae in the sample, a and $\mathrm{b}$ are coefficients of the relation, $\mathrm{y}$-intercept and slope, $\sigma$ is standard error of the residuals, $\chi_{r e d}^{2}$ is reduced chi-squared.

\begin{tabular}{|c|c|c|c|}
\hline Sample & $\mathrm{N}$ & for $t_{2}$ & for $t_{3}$ \\
\hline Parallax + RDR & 50 & $\begin{array}{l}a=-10.54 \pm 0.30 \\
b=2.04 \pm 0.23 \\
\sigma=0.9 \mathrm{mag} \\
\chi_{\text {red }}^{2}=2.14\end{array}$ & $\begin{array}{l}a=-11.46 \pm 0.42 \\
b=2.29 \pm 0.27 \\
\sigma=1.0 \mathrm{mag} \\
\chi_{\text {red }}^{2}=2.34\end{array}$ \\
\hline Parallax & 36 & $\begin{array}{l}a=-10.68 \pm 0.32 \\
b=2.11 \pm 0.25 \\
\sigma=0.8 \mathrm{mag} \\
\chi_{\text {red }}^{2}=2.30\end{array}$ & $\begin{array}{l}a=-11.64 \pm 0.46 \\
b=2.37 \pm 0.30 \\
\sigma=0.8 \mathrm{mag} \\
\chi_{\text {red }}^{2}=2.54\end{array}$ \\
\hline RDR & 32 & $\begin{array}{l}a=-11.08 \pm 0.78 \\
b=2.53 \pm 0.59 \\
\sigma=1.5 \mathrm{mag} \\
\chi_{\text {red }}^{2}=2.91\end{array}$ & $\begin{array}{l}a=-12.11 \pm 1.04 \\
b=2.80 \pm 0.67 \\
\sigma=1.5 \mathrm{mag} \\
\chi_{\text {red }}^{2}=2.64\end{array}$ \\
\hline
\end{tabular}

problem that there is considerable intrinsic scatter in the Galactic MMRD relation and this may indicate the presence of the hidden second-order parameters as mentioned in Downes \& Duerbeck (2000). Thus, we investigated $M_{V, \max }-t_{2,3}$ distributions (Fig. 2) considering other properties such as spectroscopic type, lightcurve shape, and FWHM $\mathrm{H}_{\alpha}$. The median values of the differences between the observed and the calculated absolute magnitudes, $\Delta M_{V}^{-}\left(t_{2,3}\right)$, are also given in Table 2 in which the novae are separated into samples. The median values for the $t_{2}$ and $t_{3}$ times are increasing so long as FWHM $H_{\alpha}$ gets higher. For a further investigation, we added the RN and candidate RN (cRN) sample into the MMRD distributions (Fig. 2a,d) by considering the FWHM $\mathrm{H}_{\alpha}$. Note that, we used the distances in Schaefer (2010) and Schaefer et al. (2013) for well-known Galactic RNe (RS Oph, U Sco, V3890 Sgr, and T Pyx), if it did not depend on the MMRD relation, since their distances could not be calculated from expansion parallaxes or RDRs. It is important to reveal the positions of RNe in the MMRD distributions corresponding to FWHM $\mathrm{H}_{\alpha}$, since the novae with high velocities seem to be related with RNe (Pagnotta \& Schaefer 2014). 
The absolute magnitudes of RN + cRN which have $2000 \mathrm{~km} / \mathrm{s}<$ FWHM $\mathrm{H}_{\alpha}$ are generally fainter than those obtained from MMRD relations, and the systems with $2000 \leqslant \mathrm{FWHM} \mathrm{H}_{\alpha}<3500 \mathrm{~km} / \mathrm{s}$ seem to trace different lines: $-10.03( \pm 0.45)+2.9( \pm 0.3) \log t_{2}$ and $-12.3( \pm 0.55)+3.7( \pm 0.4) \log t_{3}$. For $3500 \mathrm{~km} / \mathrm{s} \leqslant \mathrm{FWHM} \mathrm{H}_{\alpha}$, the trends are almost vertical, but we did not find significant relations from regression analysis owing to large scattering. Note that, we adopted the intrinsic errors of the distances of RNe, but the distances of some RNe differ strongly in the literature. We also tried to obtain MMRD relations by separating novae according to their light curve shape and spectral classifications, but they are all consistent with those obtained using all novae when taking into account the error of the coefficients. The following relations were obtained for samples in which number of novae is greater than 5;

- for $\mathrm{S}$ :

$$
\begin{aligned}
& -10.3( \pm 0.9)+2.1( \pm 1.1) \log t_{2} \\
& -11.3( \pm 1.3)+2.5\left( \pm 1.1 \log t_{3}\right. \\
& -11.4( \pm 1.2)+2.3( \pm 1.2) \log t_{2} \\
& -12.0( \pm 1.6)+2.2( \pm 1.2) \log t_{3} \\
& -11.3( \pm 0.7)+2.4( \pm 0.4) \log t_{2} \\
& -13.1( \pm 1.2)+3.3( \pm 0.7) \log t_{3} \\
& -10.7( \pm 0.3)+2.1( \pm 0.2) \log t_{2} \\
& -11.7( \pm 0.5)+2.4( \pm 0.3) \log t_{3} \\
& -10.6( \pm 0.8)+2.4( \pm 0.9) \log t_{2} \\
& -11.5( \pm 1.0)+2.3( \pm 0.8) \log t_{3}
\end{aligned}
$$

- for D:

- for Fe II:

- for He/N+Hybrid: $-10.6( \pm 0.8)+2.4( \pm 0.9) \log t_{2}$

The MMRD relations for subsamples are all similar with only small differences in the coefficients. Only the relation for the D-type could be slightly different one. In contrast, the novae with J-type light curves remain at a constant absolute magnitude $M_{V, \text { max }} \sim-7 \pm 0.2$ mag for 40 days $\lesssim t_{2}$ and 100 days $\lesssim t_{3}$ (Fig. 2 b,e). We also investigated the median values of the differences between the calculated and the observed absolute magnitudes (Table 4). The novae having plateaus $(\mathrm{P})$ or jitters $(\mathrm{J})$ in their light curves have brighter absolute magnitudes than that calculated from MMRD relations, while the novae with oscillations $(\mathrm{O})$ in their light curve have fainter absolute magnitudes. The other nova subsamples of smooth (S) or dips (D) light curve type are consistent when considering the stated errors. V2491 Cyg and BT Mon are the only systems with cusp (C) and flat-topped (F) light curve shape, respectively. In the following section 4.1, we investigated the $t_{2}$ and $t_{3}$ decline times for all novae in the catalogue, and derived a relation between logarithmic decline times. Moreover, we found that D and J-type novae may not have linear decline between $t_{2}$ and $t_{3}$ times. In that case there may be a dependence on the decline rates $t_{2} / t_{3}$ that changes the position of novae in these two MMRD distributions, especially for novae with D- or J-type. Note that, in the MMRD sample, there is only one nova, DK Lac, which is not in the $\pm 2 \sigma$ limits of that relation. Finally, the mean values of the differences between absolute magnitudes for the subsamples distinguished by the spectral classification are all within the stated errors, but the RNe systems in the He/N class tend to have fainter absolute magnitudes. However given the fact that the number of systems where these parameters could be determined is very small to confirm any dependence of the decline of the light curve or spectral classification (especially for $\mathrm{He} / \mathrm{N}+$ hybrid novae) on MMRD relations.

\section{NOVAE IN THE GALAXY}

In this section, the statistical results on decline times, absolute magnitudes at outburst maximum, and the spatial distribution along with Galactic scale height and space density were investigated. Using the
Table 2. The median values of the differences between the calculated and the observed absolute magnitudes for the subsample, separated according to FWHM $H \alpha$ measurements, light curve shapes (F, P, S, J, C, D), and spectral classes $(\mathrm{He} / \mathrm{N}, \mathrm{Fe}$ II, hybrid). Here, $\mathrm{N}$ is the number of novae in the sample, $\Delta \bar{M}_{V}\left(t_{2}\right)$ and $\Delta \bar{M}_{V}\left(t_{3}\right)$ are the differences of the absolute magnitudes when using $t_{2}$ and $t_{3}$ times, respectively. LF, MF and HF represent the novae with low FWHM $H_{\alpha}<2000 \mathrm{~km} / \mathrm{s}$, medium $2000 \leqslant$ FWHM $H_{\alpha}<3500 \mathrm{~km} / \mathrm{s}$ and high FWHM $H_{\alpha} \geqslant 3500 \mathrm{~km} / \mathrm{s}$, respectively.

\begin{tabular}{lcccccc}
\hline & \multicolumn{3}{c}{$\mathrm{CNe}$} & \multicolumn{3}{c}{$\mathrm{CNe}+\mathrm{RNe}$} \\
& $\mathrm{N}$ & $\Delta \bar{M}_{V}\left(t_{2}\right)$ & $\Delta \bar{M}_{V}\left(t_{3}\right)$ & $\mathrm{N}$ & $\Delta \bar{M}_{V}\left(t_{2}\right)$ & $\Delta \bar{M}_{V}\left(t_{3}\right)$ \\
\hline $\mathrm{ALL}$ & 50 & $0.20 \pm 0.14$ & $0.25 \pm 0.14$ & 65 & $0.34 \pm 0.17$ & $0.39 \pm 0.18$ \\
\hline $\mathrm{LF}$ & 13 & $0.43 \pm 0.27$ & $0.59 \pm 0.28$ & - & $0.43 \pm 0.27$ & $0.59 \pm 0.28$ \\
$\mathrm{MF}$ & 10 & $0.82 \pm 0.33$ & $1.01 \pm 0.35$ & 15 & $1.07 \pm 0.24$ & $0.98 \pm 0.25$ \\
$\mathrm{HF}$ & - & - & - & 10 & $1.24 \pm 0.78$ & $1.57 \pm 0.78$ \\
\hline $\mathrm{S}$ & 8 & $0.26 \pm 0.37$ & $0.27 \pm 0.36$ & 11 & $0.25 \pm 0.30$ & $0.36 \pm 0.31$ \\
$\mathrm{P}$ & 5 & $-0.89 \pm 0.37$ & $-0.81 \pm 0.38$ & 11 & $0.15 \pm 0.69$ & $-0.22 \pm 0.73$ \\
$\mathrm{D}$ & 12 & $-0.01 \pm 0.24$ & $0.23 \pm 0.25$ & - & - & - \\
$\mathrm{C}$ & - & - & - & 1 & 4.49 & 3.88 \\
$\mathrm{O}$ & 4 & $0.62 \pm 0.22$ & $0.56 \pm 0.20$ & - & - & - \\
$\mathrm{F}$ & 1 & 2.41 & 2.38 & - & - & - \\
$\mathrm{J}$ & 6 & $-0.52 \pm 0.31$ & $-0.62 \pm 0.28$ & - & - & - \\
\hline $\mathrm{He} / \mathrm{N}$ & 4 & $0.34 \pm 0.53$ & $0.12 \pm 0.52$ & 9 & $1.64 \pm 0.49$ & $1.11 \pm 0.45$ \\
$\mathrm{Fe} \mathrm{II}$ & 34 & $0.16 \pm 0.16$ & $0.17 \pm 0.17$ & - & - & - \\
$\mathrm{Hyb}$ & 5 & $-0.37 \pm 0.19$ & $-0.30 \pm 0.17$ & 7 & $-0.30 \pm 0.14$ & $-0.30 \pm 0.13$ \\
\hline
\end{tabular}

results obtained in this section, we also estimated the nova rate for disk novae.

In further analyses, we divided the sample into two subsamples corresponding to the used calculation method of the distance and/or absolute magnitude at maximum outburst.

- Sample 1: Only the novae with reliable distance measurements as listed in Table 5 were used.

- Sample 2: For the novae in this sample, only the MMRD relations for $t_{3}$ following $t_{2}$ times were used, assuming that the MMRD relation derived in Section 3 is correct.

2a: The novae in this sample have reliable light curve parameters. $2 \mathrm{~b}$ : The novae in this sample have unreliable light curve parameters

Note that, we did not use the MMRD relations for $22 \mathrm{RN}$ and cRN (including likely RN systems with high FWHM $\mathrm{H}_{\alpha}>3500 \mathrm{~km} / \mathrm{s}$ ) owing to deviations from the MMRD relations.

\subsection{Decline Times and Absolute Magnitudes}

The decline from outburst maximum is generally smooth, $38 \%$ of the novae (Strope et al. 2010), but a considerable number of novae shows jitters, oscillations, dips or flares in their light-curve. This heterogeneity in nova light curve shapes is a result of the variety of nova outbursts, and it may cause a non-linear decline even at relatively stable decline times ( $t_{2}$ and $t_{3}$ ). The non-linear decline of the light curve also affects calculations of absolute magnitudes from MMRD relations. Because of these reasons, we compared decline rates by considering the light curve shape classification (Strope et al. 2010) in Fig. 3. The median of differences between logarithmic decline times, and between absolute magnitudes calculated from MMRD relations for $t_{2}$ and $t_{3}$ times are listed in Table 3. From the distribution of decline times, a relation between $t_{2}$ and $t_{3}$ times were obtained as $\log t_{3}=0.91( \pm 0.02) \times \log t_{2}+0.41( \pm 0.02)$ 

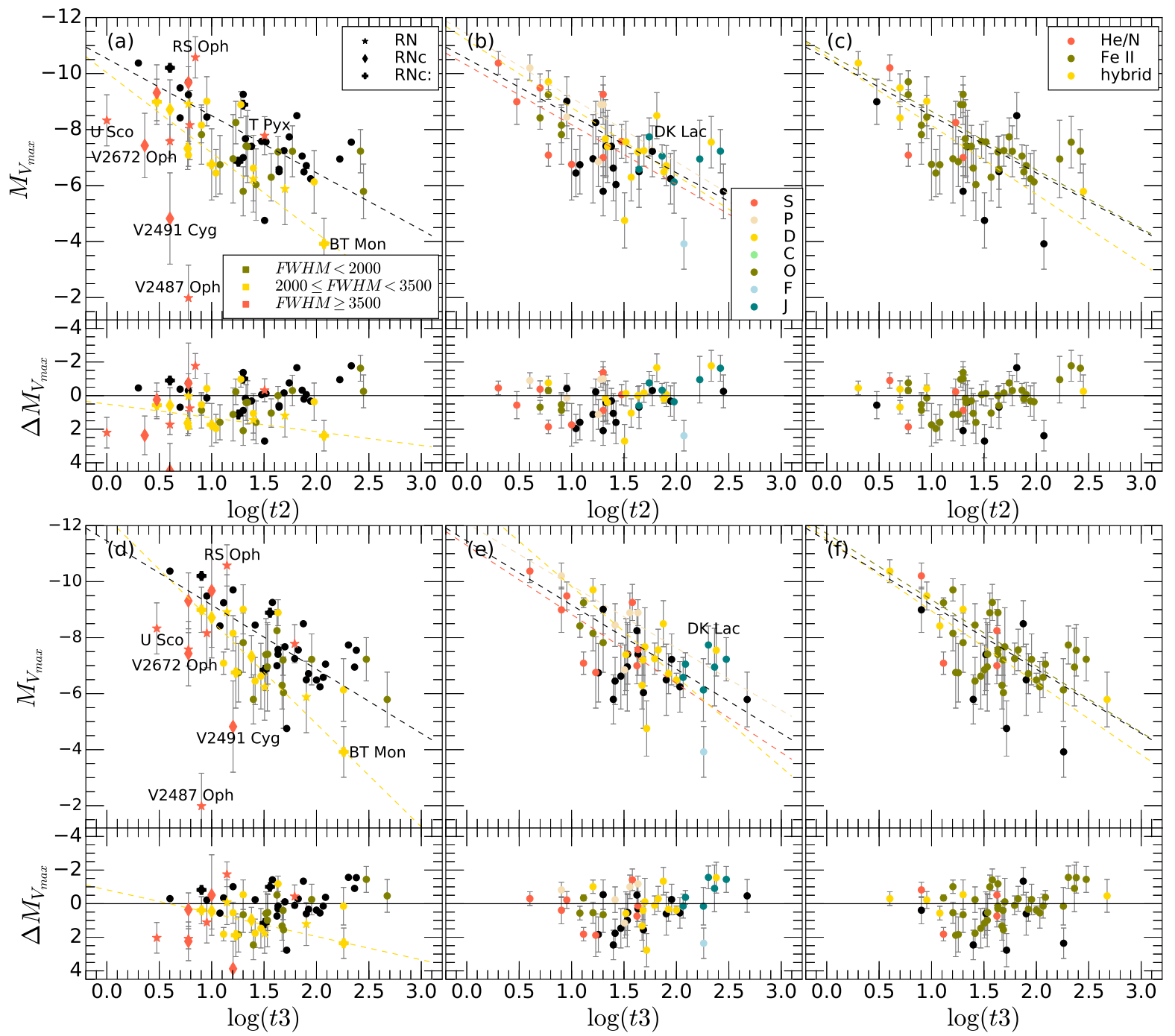

Figure 2. $M_{V, \text { max }}-t_{2,3}$ distributions of Galactic novae: (a,b,c) and (d,e,f) are for $t_{2}$ and $t_{3}$, respectively, (a,d) considering FWHM $\mathrm{H}_{\alpha}$ and including RN $+\mathrm{RN},(\mathrm{b}, \mathrm{e})$ considering the classification of light curve shapes, and (c, f) considering the spectral classification. Black lines represent the adopted MMRD relation. We denoted the trends in the distributions by considering their FWHM $\mathrm{H}_{\alpha}$ velocities, light curve shapes and spectral classification as colored dashed lines, as defined in legends. The residuals are shown in the lower panel. Recurrent novae and relatively scattered novae are labeled. DK Lac changes its position on these MMRD distributions owing to a non-linear decline.

with $R^{2}=0.93$ and one standard deviation error of \pm 0.12 day. Our relation between decline times is consistent with that found by Warner (1995), $\log t_{3}=0.88 \times \log t_{2}+0.44$. Here, the intercept value of 0.91 implies that the novae have nearly linear declines between the $t_{2}$ and $t_{3}$ times. However, 12 of 192 novae have larger separations between the logarithmic $t_{2}$ and $t_{3}$ times (Fig 3 ), especially for X Cir, V2362 Cyg and V1039 Cen. For these scattered novae, the differences between absolute magnitudes from MMRD relations for $t_{2}$ and $t_{3}$ are larger than $\pm 2 \sigma \sim 0.5 \mathrm{mag}$. Note that these systems were excluded from the samples for spatial distribution analyses. On the other hands, we also obtained relations between decline times for subsamples of light curve shape classifications, which have more than 5 systems, as following;

- For S-types: $\log t_{3}=0.96 \log t_{2}+0.32, R^{2}=0.95, \sigma=0.04$

- For P-types: $\log t_{3}=0.92 \log t_{2}+0.43, R^{2}=0.90, \sigma=0.07$

- For D-types: $\log t_{3}=0.72 \log t_{2}+0.60, R^{2}=0.93, \sigma=0.05$

- For J-types: $\log t_{3}=0.46 \log t_{2}+1.29, R^{2}=0.57, \sigma=0.12$
The relations for S- and P-types are very similar to those obtained from all systems, but the novae having D-and J-types seem to follow different trends that indicate these systems may not have non-linear declines. However, these scattered novae cover only $1 \%$ of the whole sample, and the mean of differences between absolute magnitudes (Table 3) are the within limits of $\pm 2 \sigma=0.5 \mathrm{mag}$. Thus, we assumed this effect is negligible in the calculation of the absolute magnitudes from MMRD relations for the $t_{2}$ and $t_{3}$ times.

The absolute magnitude of the Galactic novae, excluding $\mathrm{RN}+\mathrm{cRN}$, typically range from $M_{V_{\max }} \simeq-5$ to $-10 \mathrm{mag}$. For the novae in sample 2, the histogram distribution could be fitted with a Gaussian distribution with a peak at $M_{V_{\max }} \simeq-7.9 \pm 0.9$ mag. Even though, when the novae only with reliable light curve (sample 2a) are considered, we get the same result for the Gaussian peak. This value is consistent with Shafter et al. (2009) where the mean peak absolute magnitude for Galactic novae was obtained to be $7.8 \mathrm{mag}$, but smaller than their estimate of $\mathbf{- 7 . 2}$ mag for M31. For the novae in sample 1, the mean absolute magnitude changed to 


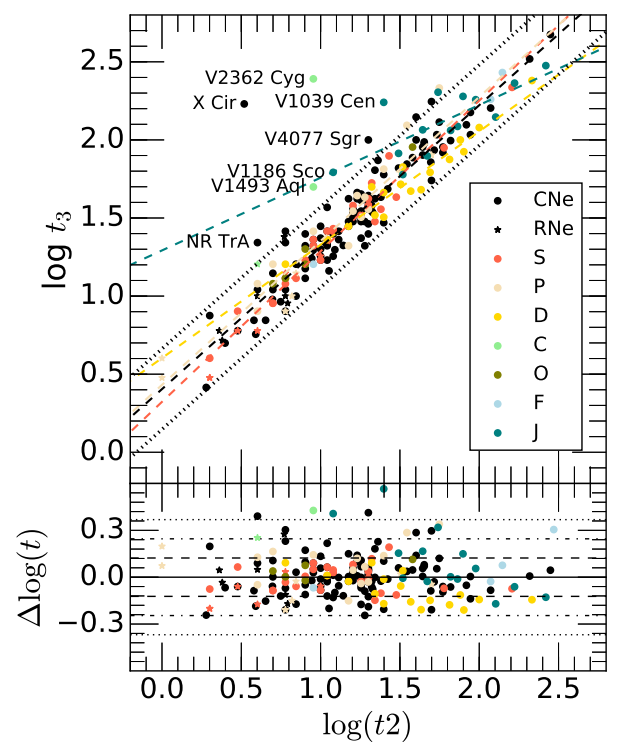

Figure 3. The distributions of $t_{2}$ vs $t_{3}$ decline times. Dashed lines represent the relations between decline times corresponding to subsamples by light curve shapes. The dotted lines are for the $\pm 2 \sigma$ ranges from the relation obtained for all novae in the sample.

Table 3. Comparison of logarithmic decline times, and absolute magnitudes. $\mathrm{N}$ is number of novae in the sample, $\Delta t$ is residuals, $\log \left(t_{3}\right)-\log \left(t_{3, c}\right)$ ), $\Delta M_{V}\left(t_{2}, t_{3}\right)$ is median of differences between absolute magnitudes obtained from MMRD relations for $t_{2}$ and $t_{3}$ times.

\begin{tabular}{l|ccc|cc}
\hline Sample & \multicolumn{3}{|c|}{$\mathrm{CNe}$} & \multicolumn{2}{c}{$\mathrm{CNe}+\mathrm{RNe}$} \\
& $\mathrm{N}$ & $\Delta t$ & $\Delta M_{V}\left(t_{2}, t_{3}\right)$ & $\mathrm{N}$ & $\Delta t$ \\
\hline All & 192 & $-0.0 \pm 0.01$ & $0.06 \pm 0.03$ & 214 & $0.0 \pm 0.01$ \\
$\mathrm{~S}$ & 26 & $0.00 \pm 0.02$ & $0.04 \pm 0.04$ & 32 & $-0.03 \pm 0.02$ \\
$\mathrm{P}$ & 13 & $0.12 \pm 0.04$ & $0.32 \pm 0.08$ & 21 & $0.05 \pm 0.03$ \\
$\mathrm{D}$ & 17 & $-0.11 \pm 0.02$ & $-0.11 \pm 0.02$ & - & - \\
$\mathrm{C}$ & 2 & $0.77 \pm 0.24$ & $1.82 \pm 0.56$ & 3 & $0.43 \pm 0.22$ \\
$\mathrm{O}$ & 5 & $0.04 \pm 0.02$ & $0.13 \pm 0.05$ & - & - \\
$\mathrm{F}$ & 4 & $0.02 \pm 0.07$ & $0.15 \pm 0.17$ & 4 & $0.02 \pm 0.07$ \\
$\mathrm{~J}$ & 14 & $0.03 \pm 0.05$ & $0.15 \pm 0.12$ & - & - \\
\hline
\end{tabular}

$M_{V_{\text {max }}} \simeq-7.2 \pm 0.24$ mag, which is consistent with that adopted by Shafter (2017), but the histogram distribution is broad without a significant peak. The maximum magnitudes mainly depend on the discovery magnitudes so that the absolute magnitude at maximum is likely underestimated. Interstellar extinction obscure relatively faint Galactic novae, so the mean absolute magnitude at outburst maximum for the Galaxy could be biased towards brighter novae, corresponding to the Galactic disk population in Solar neighbourhood. In contrast, as with the case of both novae V598 Pup and KT Eri (Hounsell et al. 2010a), the brightest novae that can even be seen by naked eye, may not be discovered unless they are monitored in different phases such as X-ray, and be missed by conventional ground-based observing techniques. Thus the Galactic (and extra galactic) nova sample is not free from selection effects that likely bias the results.

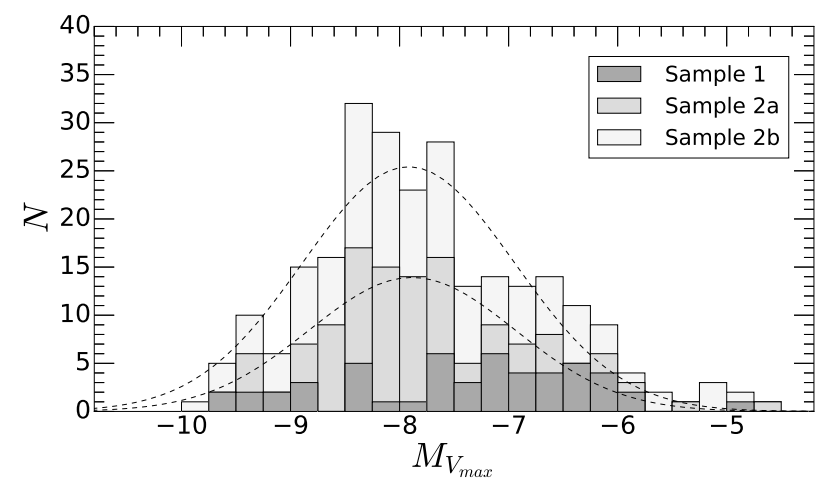

Figure 4. Absolute magnitude distribution of the Galactic nova samples. The distributions are fitted by Gaussian functions which are represented by dashed lines.

\subsection{Spatial Distributions}

The Galactic distribution of all Galactic novae in our catalogue is given in Fig. 5. In this distribution, systems are mainly located at low Galactic latitudes $\left(b \leqslant 10^{\circ}\right)$ especially towards the Galactic bulge. Since the Galactic novae in the sample concentrate in the Galactic plane, where the bulk of the absorbing medium is located, the extinction for many of them can be high and can not be neglected. Although, interstellar reddening estimates were given for nearly half of the novae in the catalogue, the extinction and distances for the remaining novae were simultaneously determined by an iteration method: We first obtained the interstellar extinction for each distance step in the line of sight following Sharma et al. (2011) and Binney et al. (2014). We then calculated the absolute magnitude of each nova from the MMRD relation. Using the apparent magnitude and the absolute magnitude we can calculate the corresponding possible distance extinction values for each nova. We estimated the distance and the extinction of each nova simultaneously by calculating the point where the distance and the extinction derived from the distance modulus $\left(V_{\max }-M_{V_{\max }}=5 \operatorname{logd}-5+A_{V}\right)$ matches the values derived from the dust extinction model. To test the robustness of our method we also followed this method for the novae which already have independent extinction measurements. The standard deviation of the differences between the reddening derived from the method summarized above to the independent measurements is found to be $\pm 0.08 \mathrm{mag}$. We used this value as our error on determining the interstellar reddening towards the sources which have no independent reddening measurements. Using this iteration method with the MMRD relation only for the novae without reddening estimates in sample 2 provides us a large sample to investigate the spatial distribution and Galactic model parameters of Galactic novae.

To inspect the Galactic distribution of Galactic novae, heliocentric distances (X towards Galactic centre, $\mathrm{Y}$ towards the Galactic rotation, $\mathrm{Z}$ towards the Galactic north Pole) are obtained, and the projected positions on the Galactic plane (XY) and on the plane perpendicular to it (XZ) are derived (Fig.6). The Galactic novae in our sample are mainly concentrated in the Galactic disk (Fig. 6b) and towards the inner Galaxy (Fig. 6a), but the novae in the neighborhood of the Galactic center are located at the edge of bulge. The novae in sample 1 occupy a smaller spatial volume than the novae in sample 2 where a number of novae (11 of 120) with uncertain light curve novae in sample 2 has unrealistic distances (>20 kpc) even beyond the Galactic bulge through Galactic disk. To avoid such 


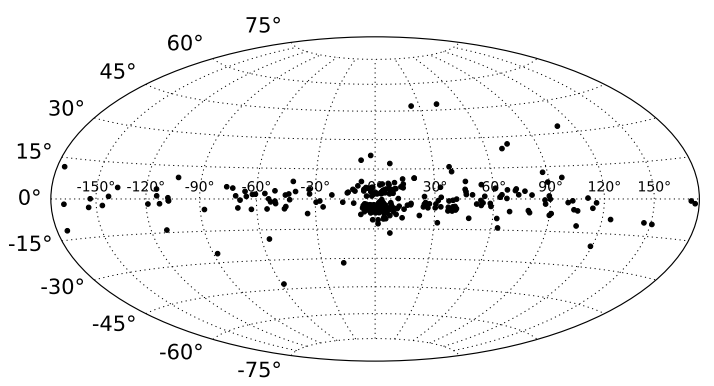

Figure 5. Galactic coordinates of novae in our sample.

unrealistic measurements, we set an upper limit on the distance calculations from MMRD relations of $20 \mathrm{kpc}$. The median values of XYZ distances for sample 2 are $4.30,0.39$ and $-0.13 \mathrm{kpc}$, while the novae in sample 1 have about 1.0, 0.5, and $0.02 \mathrm{kpc}$, and these do not change even with adding $\mathrm{RNe}$. The numbers of systems with $(X, Y, Z) \geqslant 0$ are $(52,43,30$ of 65$)$ for sample $1,(66,53,46$ of 80 ) for sample 1 when adding RNe, and $(218,156,100$ of 249) for sample 2. In short, $\sim 85 \%, \sim 65 \%$ and $\sim 50 \%$ of Galactic novae in samples have Galactic distances of $(X, Y, Z) \geqslant 0$, respectively. The results indicate that there is a strong bias towards the Galactic bulge. Since the sky surveys are mainly concentrated towards the Galactic bulge, this bias probably arises from observational selection effects. Although, the number of the bulge novae is expected to be more than half of all novae as in M31 (Darnley et al. 2006), the Galactic novae in our sample are mainly located on the edge of the Galactic bulge likely due to increasing interstellar reddening effect towards the Galactic center. This distribution indicates that the bulge novae could not be observed in our sample.

In the studies of the spatial distribution of the novae, the thin disk population is characterized by bright and fast novae, while slow and faint novae belong to the thick disk or bulge population. The differences between the two populations are explained by the different nature of the nova progenitors (della Valle et al. 1992a). Fast novae characterized as $\mathrm{He} / \mathrm{N}$ novae are believed to be associated to relatively massive WDs, while slow novae related to Fe II novae have less massive WDs. Since more massive WD requires smaller mass of the accreted material to start thermonuclear runaway, the more violent outburst theoretically occurs with higher expansion velocity and fast decline. Hence, these novae with fast decline time related to $\mathrm{He} / \mathrm{N}$ evolve more quickly, and they should be located closer to the Galactic plane. Della Valle \& Livio (1998) proposed that fast novae $\left(t_{3}<20\right.$ day) belonging to the He/ $\mathrm{N}$ spectroscopic class are preferentially concentrated through Galactic plane $(Z<$ $100 \mathrm{pc}$ ) related to Pop I, while Fe II novae with slow decline time $\left(t_{3}>20\right.$ day) are located up to $>1000 \mathrm{pc}$ from the Galactic plane, and they are likely related to the thick disk/bulge (Pop II) population. To test this prediction, we considered the histogram distribution of these systems based on their spectral and speed class. In the samples, $\sim 80 \%$ of Fe II novae (72 of 87) are slow novae $\left(t_{3} \geqslant 20\right.$ days), while the percentage of fast novae with $t_{3}<20$ days belonging to the He/N+hybrid class is $~ 60 \%$ (11 of 18)). Note that we considered the hybrid class sources similar to $\mathrm{He} / \mathrm{N}$ novae for the analyses. Fig. 7 presents the histogram distribution of the $t_{3}$ times according to the spectral classification, where both Fe II and $\mathrm{He} / \mathrm{N}+$ hybrid novae are scattered throughout the whole range of $t_{3}$ times, but the peak values of the histogram distribution for Fe II and

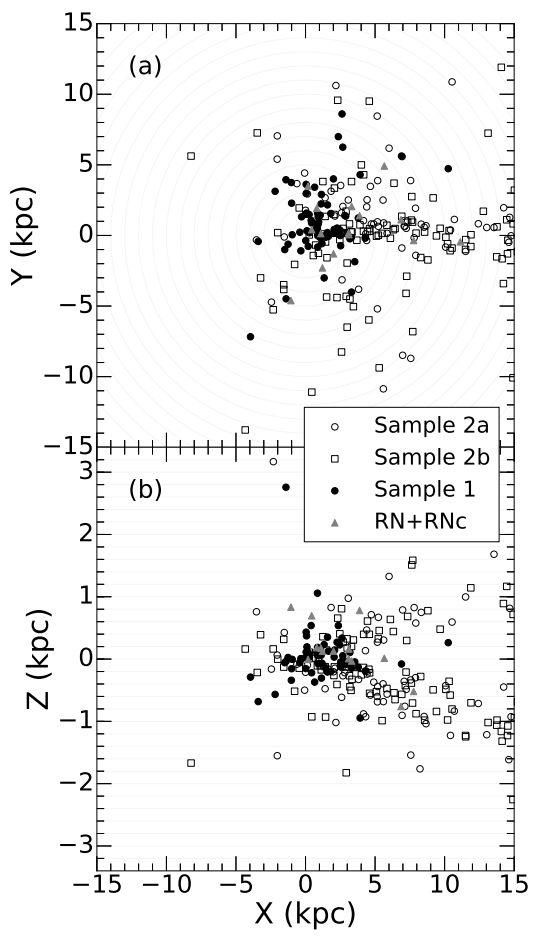

Figure 6. The projected positions of novae in our sample (a) on the X-Y Galactic plane and (b) on X-Z plane perpendicular to it.

$\mathrm{He} / \mathrm{N}+$ hybrid are at $t_{3}=37$ and 13 days, respectively. The vertical Z-distances for their spectral classes (Fig. 8a) indicate that vertical distances for $\mathrm{Fe}$ II and $\mathrm{He} / \mathrm{N}+$ hybrid novae in sample 2 extend up to $\sim 3.0$ and $\sim 1.7 \mathrm{kpc}$ from the Galactic plane with median values of vertical Z-distances of 0.3 and $0.33 \mathrm{kpc}$, respectively. Nine of Fe II novae (10\%) were found higher than $1000 \mathrm{pc}$ above the plane, and 16 of Fe II novae (18\%) are located in the Galactic plane $(Z<100 \mathrm{pc})$. For the He/N+Hybrid novae, the number of systems with $Z>1000 \mathrm{pc}$ is only $1(6 \%)$, and 6 of them (33\%) are at $Z<100 \mathrm{pc}$. The median Z-distances changes to 0.15 and $0.03 \mathrm{kpc}$ for the novae in sample 1 ( 0.15 and $0.12 \mathrm{kpc}$ with adding $\mathrm{RNe})$, but the novae classified as hybrid class in both sample 1 and 2 are at shorter vertical Z-distances. Since most of the novae in sample 1 are nearby (Fig.6) as mentioned above, their Z-distances extend only up to $1 \mathrm{kpc}$. The distributions of $\mathrm{Z}$ distances according to fast and slow novae show similar trends as derived for the spectral classification. Moreover, both slow and fast novae in sample 2 (Fig 8) are located up to $\sim 4 \mathrm{kpc}$ from the Galactic plane. Large part of fast novae (11 of $51,22 \%$ ) and only 25 of 189 slow novae (13\%) have vertical distances from the Galactic plane higher than $1000 \mathrm{pc}$, while only 7 of 51 fast novae (14\%) and 23 of 189 slow novae (12\%) lie in the Galactic plane at $Z<100 p c$. These trends do not change even for the novae in sample 1 when adding RN+cRN. Although, Della Valle \& Livio (1998) concluded that spectral or speed classification correlates with the stellar population. Such relations were not derived from our samples which contain much more novae with spectral classification. On the contrary, both $\mathrm{Fe}$ II and $\mathrm{He} / \mathrm{N}$ novae or fast and slow novae are distributed in the whole range of vertical distances, but are mainly concentrated throughout the Galactic disk. However, a similar selection bias (as mentioned in section 4.1) may effect this result as well. The Galactic nova samples seem to contain the systems, which are located in the Galactic disk structure related to Pop I or young Pop II. The faint/undiscovered novae belongs to 


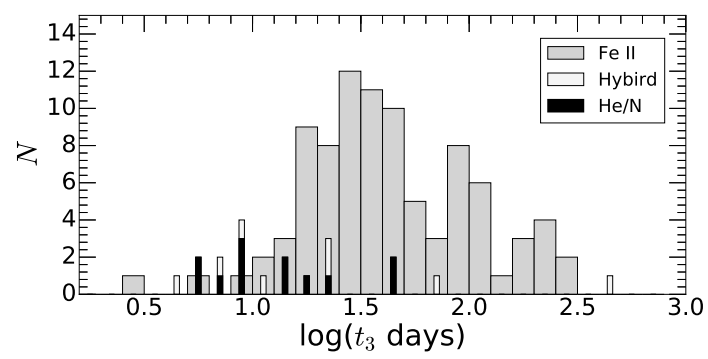

Figure 7. The histogram distributions of decline times for the Galactic novae corresponding to their spectral classes.

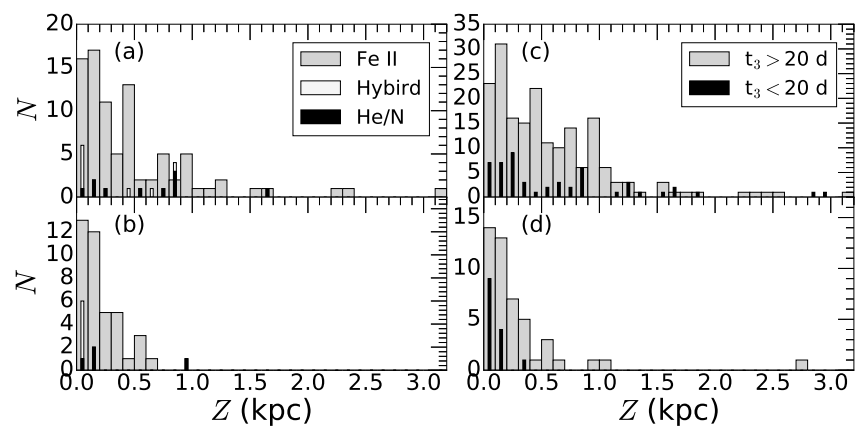

Figure 8. Z-distance histogram distributions of the Galactic novae corresponding to their $(a, b)$ spectral and $(c, d)$ speed classes, $(a, c)$ for the novae in sample $2,(b, d)$ for the novae in sample 1 .

thick disk or halo components may show a correlation with spectral or speed classifications.

\subsection{Galactic Model Parameters of the Galactic Novae}

In this section, we investigate the Galactic model parameters of the Galactic novae such as the scale height and space density. Our sample contains novae erupted as far back as the 19th century resulting in a sample covering $\sim 200 \mathrm{yr}$ of observations, but the systematic and effective discoveries of novae have only been possible since 1960s so the effective observing time of roughly 50 years is used in the following calculations. Since only the novae with observed outbursts are included in the sample, there is a completeness problem arising from unrecorded nova outbursts due to all kind of observational effects. This incompleteness hardly influences the vertical spatial $(\mathrm{Z})$ distribution but strongly affects the space density as mentioned in previous studies (Patterson 1984; Duerbeck 1984; della Valle \& Duerbeck 1993; Shafter 2017). Thus, the space density $\rho_{0}$ can only be calculated from the observed local outburst density $\rho_{\text {out }}(0)$ for the discovered classical nova sample by taking into account the recurrence time $\left(T_{R}\right) ; \rho_{0}=\rho_{\text {out }}(0) \times T_{R}$. A detailed discussion of both the space density and the recurrence time calculation in previous studies were given in della Valle \& Duerbeck (1993), which was the last study on the space density of the Galactic nova population.

The recurrence time of classical novae, which may range from a few hundred to thousand years, depends on several factors, for example the mass of the WD, the donor star, the amount of ejected material, or the rate of mass transfer. It even changes with the stellar population and the type of the host galaxy (Chen et al. 2016). However, the mean recurrence times for classical novae were es- timated to be between $T_{R}=3000-26000$ years (Patterson 1984; Duerbeck 1984; della Valle \& Duerbeck 1993). Since the calculated recurrence times show a very large scatter, the space density determined in this way will be only a limit for the actual value. Thus, we adopted two recurrence times as 3000 and 26,000 years to set a lower and upper boundary on the space density for classical novae.

On the other hand, in order to be able to determine the Galactic model parameters, the Galactic novae should be separated into samples based on stellar populations. Although not very effective, the only way to estimate the stellar population is to deduce Z-distances. In our sample, $82 \%$ of the novae have vertical distances smaller than $0.825 \mathrm{kpc}$. Note that as discussed below, for the sake of completeness we only used the novae with distances up to $4 \mathrm{kpc}$ in our further estimations, and $99 \%$ of these novae have Z-distances smaller than this limit. Here, the vertical distance of $0.825 \mathrm{kpc}$ is important, since the spatial densities of thin and thick disks are equal at this mode value of the vertical distance (Ak et al. 2015). Thus, almost all Galactic novae in the sample can be considered as members of the thin-disk component of the Galaxy. This is a reasonable assumption because the population analysis already showed that $94 \%$ of cataclysmic variables in the Solar neighbourhood belong to the thin-disk component of the Galaxy (Ak et al. 2015). The contribution of thick disk or halo CVs to the thin disk sample adds a small effect (less than 4\%) to the scale height estimations Özdönmez et al. (2015). In this case, the effect of contribution can be considered as negligible. Thus, Galactic novae in our sample were not classified based on the population types in the estimation of the Galactic model parameters and all are assumed to be thin disk members.

The local outburst density can be obtained using the Galactic novae within a cylinder centered at the Sun with a radius and infinite height. Duerbeck (1984) used a cylinder radii of $d_{X Y}=1000 \mathrm{pc}$ and assumed that all the novae in this region are already known. This is a reasonable assumption, since the completeness of the novae decreases after this radius where the novae at the edge reach to an apparent magnitude of 4 to 5 mag. In Figure 9a,d, the distribution of the z-distances of the novae in our sample are given as a function of their $d_{X Y}$ distances. This distribution implies that the novae with apparent magnitude brighter than 3 and 5 mag reach to $d_{X Y}=2$ and $4 \mathrm{kpc}$, but most of them are concentrated within $2 \mathrm{kpc}$. The cumulative number distribution of the novae in different cylinders with radii $d_{X Y}$ are given in Fig. 9b,e. The number of the discovered novae should increase with larger radius, but this trend slows after $2 \mathrm{kpc}$, and it becomes almost constant after $\sim 4 \mathrm{kpc}$. Since the discovery efficiency has increased in last decades, it is possible to assume a larger radii than that adopted by Duerbeck (1984) within which all the possible classical novae have already been discovered. From the classical novae sample 1 and 2 within the cylinder with $d_{X Y}=2 \mathrm{kpc}$, the outburst space density in the solar neighborhood is calculated as $\rho_{\text {out }}(0)=3.57 \pm 0.25$ and $\rho_{\text {out }}(0)=$ $4.22 \pm 0.25 \times 10^{-10} \mathrm{pc}^{-3} \mathrm{yr}^{-1}$ with a scale height of $148 \mathrm{pc}$ and $175 \mathrm{pc}$ (see Figure 9c,f), respectively. The results of the calculations are given in Table 4 . If $4 \mathrm{kpc}$ is adopted as the radius of the cylinder, the outburst density can be found to be as 1.3 and $1.47 \times 10^{-10}$ $\mathrm{pc}^{-3} \mathrm{yr}^{-1}$ with a scale height of $\sim 165 \mathrm{pc}$ and $\sim 209 \mathrm{pc}$ for the classical novae in sample 1 and 2 , respectively. Using $1 \mathrm{kpc}$ radius, we calculated $\rho_{\text {out }}=6.05 \times 10^{-10} \mathrm{pc}^{-3} \mathrm{yr}^{-1}$ for only the novae in sample 2. In our analyses, the radii of the cylinder and/or using different samples, depending on reliable distance measurements (sample 1) or the MMRD relation (sample 2), did not significantly change the local outburst density. So even if a MMRD relation is not realized, our result is consistent. It is in the range of $1.3-6 \times 10^{-10}$ $\mathrm{pc}^{-3} \mathrm{yr}^{-1}$. Our result is slightly larger than previous observational 
Table 4. Result for spatial distribution analyses, $d_{X Y}$ is radius of cylinder, $\rho_{\text {out }}$ is the local outburst space density, $\mathrm{H}$ is the scale height, $\rho_{0}$ is the local space density using $T_{R}=3000$ and $23000 \mathrm{yr}^{-1}$.

\begin{tabular}{ccccccc}
\hline & $\begin{array}{c}d_{X Y} \\
(\mathrm{kpc})\end{array}$ & $\begin{array}{c}\rho_{\text {out }} \\
\left(\times 10^{-10} \mathrm{pc}^{-3} \mathrm{yr}^{-1}\right)\end{array}$ & $\begin{array}{c}H \\
(\mathrm{pc})\end{array}$ & $\begin{array}{c}\rho_{0} \\
\left(\times 10^{-6} \mathrm{pc}^{-3} \mathrm{yr}^{-1}\right) \\
T_{R}=3000\end{array}$ & $\begin{array}{c}T_{R}=26000 \\
\left(\mathrm{yr}^{-1}\right)\end{array}$ \\
\hline Sample 1 & 2 & $3.57 \pm 0.25$ & $148 \pm 17$ & 1.07 & 9.3 & $54_{-13}^{+16}$ \\
& 1 & - & - & - & - & - \\
& 4 & $1.30 \pm 0.13$ & $165 \pm 21$ & 0.39 & 3.4 & $23_{-7}^{+9}$ \\
\hline Sample 2 & 2 & $4.22 \pm 0.26$ & $175 \pm 17$ & 1.3 & 11.0 & $80_{-16}^{+21}$ \\
& 1 & $6.05 \pm 1.46$ & $147 \pm 62$ & 1.8 & 15.7 & $95_{-65}^{+92}$ \\
& 4 & $1.47 \pm 0.11$ & $209 \pm 22$ & 0.4 & 3.8 & $35_{-8}^{+9}$ \\
\hline
\end{tabular}

estimates, cf. $1.7 \times 10^{-10}$ (Patterson 1984), $3.8 \times 10^{-10}$ (Duerbeck 1984), $1-4.4 \times 10^{-10}$ (Naylor et al. 2009), $0.30 \times 10^{-10} \mathrm{pc}^{-3} \mathrm{yr}^{-1}$ (della Valle \& Duerbeck 1993), as well as the semi-model estimate of $\sim 1 \times 10^{-10} \mathrm{pc}^{-3} \mathrm{yr}^{-1}$ for disk novae (Shafter 2017). Using $T_{R}=3000$ and $26,000 \mathrm{yr}$, the space density is calculated as 1.07 $\times 10^{-6}$ and $9.3 \times 10^{-6} \mathrm{pc}^{-3}$ for the classical novae in sample 1 within the cylinder with a radius of $d_{X Y}=2 \mathrm{kpc}$, while for the novae in sample 2, it is calculated as $1.3 \times 10^{-6}$ and $11.0 \times 10^{-6} \mathrm{pc}^{-3}$. Taking into account all calculations for all samples and cylinder radii, the range of the space density of novae is obtained as $0.4-15.7 \times 10^{-6}$ $\mathrm{pc}^{-3}$.

Computing the local outburst density of Galactic novae corresponding to the Galactic disk allows us to estimate the disk nova rate. For calculations, we used the same assumptions and directextrapolation method as described in Shafter (2017), who calculated the overall Galactic nova rate by extrapolating the nova rate (assuming different observed rate for novae reaching $m \leqslant 2$ ) for a given apparent magnitude to include the entire Galaxy based on Bahcall \& Soneira (1980)'s models of bulge and disk distributions. Since we calculated the local outburst density and the scale height observationally, we only changed these parameters as given in table 4, while we adopted mean values of absolute magnitude of $-7.2 \pm 0.24$ and $-7.9 \pm 0.9$ mag as calculated for sample 1 and 2, respectively, in section 4.1. The results are given in Table 4 that shows disk nova rates are in the range of $\sim 20$ to $\sim 100$ per year. An average of the calculated plausible parameters, $\rho_{\text {out }}=3.9 \pm 0.25 \times 10^{-10} \mathrm{pc}^{-3}$ $\mathrm{yr}^{-1}, H=160 \mathrm{pc}, M_{V \max }=-7.5 \pm 0.9$ mag, yields a disk nova rate of $N_{\text {rate }}=67_{-17}^{+21} \mathrm{yr}^{-1}$. Our results are slightly larger than the results adopted by Shafter (2017). They calculated disk nova rates in the range of $\sim 35$ to $\sim 110$ per year by assuming different observed rate of the novae with apparent magnitude less than 2 mag and different mean absolute magnitudes (similar values as in our study) for Galactic disk novae. They adopted $50_{-23}^{+31} \mathrm{yr}^{-1}$ as a best estimate for an average of global nova rates (bulge + disk), where the disk nova rate was calculated as $45_{-21}^{+27}$ per year. Our average estimate is slightly larger than the average estimate for the disk nova rate in Shafter (2017), but it is consistent within errors. We should note that the relatively larger nova rate calculated here is probably arising from using a larger local outburst density. However, if the rate of undiscovered novae is higher than expected, both local outburst density and the nova rate would be increase to larger values. For example, our result would be consistent with Shafter (2017), if observed rate of novae (with $m \leqslant 2$ ) assumed between $43 \%-90 \%$.

\section{RESULTS}

In this study, we investigated the MMRD relation and spatial distributions of Galactic novae from a new compiled catalogue. The catalogue, which will be updated frequently, has fundamental parameters, but more importantly it contains the reddening and distance estimates from the improved RDRs. We continue to collect the related parameters, and study the Galactic novae spectroscopically to obtain their interstellar reddening/extinctions that allow us to limit their distances, and investigate their spectroscopic features. In addition, the distances in GAIA DR2 will be added to the catalogue. Hence, this catalogue may help to resolve uncertain distances of some nova systems, which have lot of variety. By this way, the nature of the novae in the Galaxy might be clarified in the future. In our (spatial) analyses, we used two different samples. The first sample contains the novae whose distance measurements (non-MMRD) exist, while we only used MMRD relations for the second sample. Main results of our paper can be summarized as follows.

- Using the distances in the catalogue, we obtained MMRD relations for $t_{2}$ and $t_{3}$ decline times with an scatter of $\sim 1$ mag. We suggest that there is a possible dependence of light curve shapes and $\mathrm{FWHM} \mathrm{H}_{\alpha}$ on MMRD relations.

- The results obtained by using the two different samples for the spatial analyses are consistent with each other. Thus, we confirm that the MMRD relation is still useful tool for statistical analyses.

- The distributions of decline times $t_{2}$ and $t_{3}$ indicate that there is a relation between decline times, but it depends on light curve shape classifications, especially for D- and J-types.

- From the histogram distributions of mean absolute magnitude at outburst maximum, we obtained $M_{V_{\max }}=-7.2 \pm 0.24$ and $-7.9 \pm 0.9$ mag for the samples.

- The spatial distributions of discovered Galactic novae imply that the systems are mainly placed in the Galactic disk towards the Galactic bulge, but belong to the disk population. Besides, we did not found any correlation of the stellar population with spectral or speed classification.

- For the calculation of the Galactic model parameters, we used the novae within cylinders with various radii. The best estimates for the local outburst density are calculated as 3.6 and $4.2 \times 10^{-10}$ $\mathrm{pc}^{-3} \mathrm{yr}^{-1}$ with a scale height of 148 and $175 \mathrm{pc}$ for two different samples, while a possible range is $1.3-6 \times 10^{-10} \mathrm{pc}^{-3} \mathrm{yr}^{-1}$. Using $T_{R}=3000-26000 \mathrm{yr}$, the most plausible range for the local space density is calculated as $1-10 \times 10^{-6} \mathrm{pc}^{-3}$.

- A simple calculation on disk nova rate suggest that it is in range of $\sim 20$ to $\sim 100 \mathrm{yr}^{-1}$ with an average estimate $67_{-17}^{+21}$.

\section{DISCUSSIONS AND CONCLUSIONS}

Based on a new Galactic nova catalogue, the MMRD relation and the spatial distribution of novae are investigated. The MMRD relation is an effective tool (in fact it is the only one) when it is applied in a statistical way to a large sample, but it is not very reliable for individual novae because of the large individual errors and scatter in the overall relation. For a single nova, solely relying on this relation, the absolute magnitude may differ $\sim 1$ mag from the actual value. In order to consider any possible systematic effect on the MMRD relations due to the distance measurement method, MMRD relations for three different samples distinguished by distance measurements methods were obtained, but we did not find any significant bias, and we adopted the MMRD relation that depends on the novae 

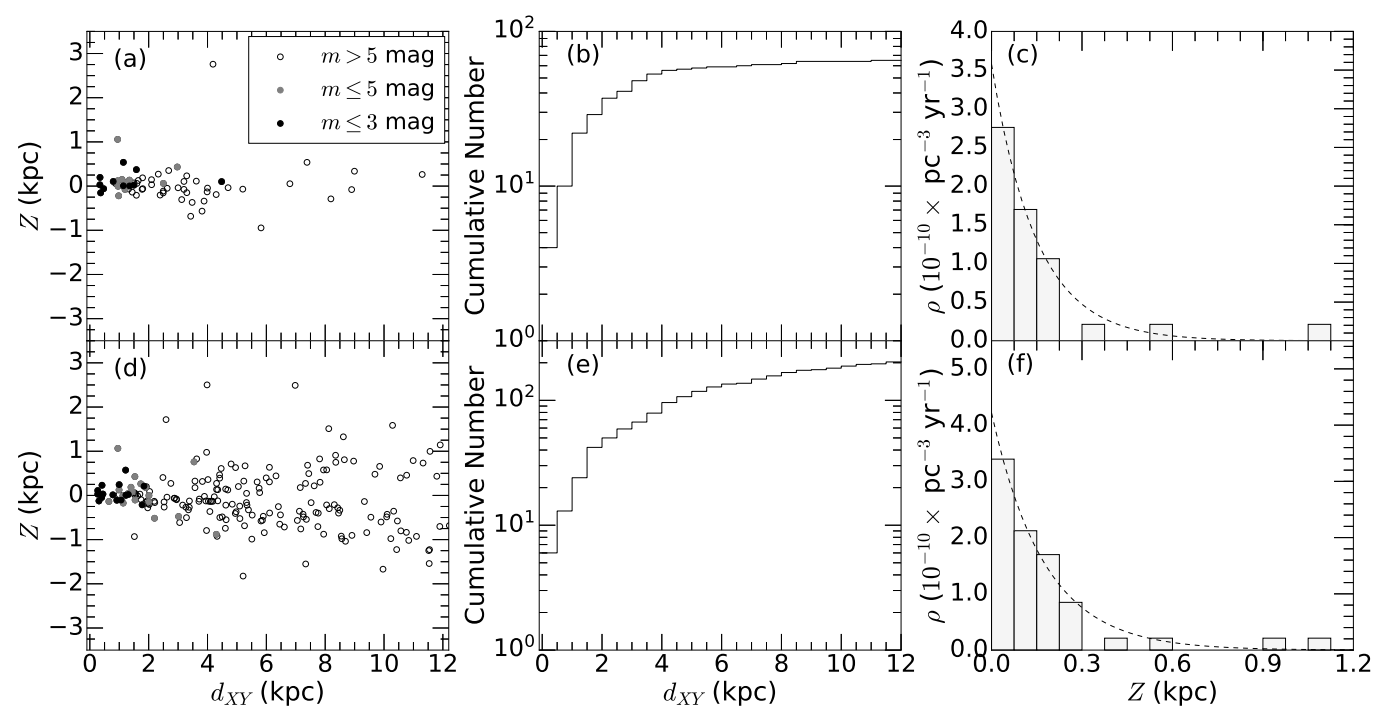

Figure 9. (a,d) Z-distance of the Galactic novae in the XY direction, (b, e) Cumulative numbers of the Galactic novae occupying a cylindrical volume of with radius $d_{X Y},(\mathrm{c}, \mathrm{f})$ the outburst density in the z-direction within a cylinder with a radius of $d_{X Y}=2 \mathrm{kpc},(\mathrm{a}, \mathrm{b}, \mathrm{c})$ for $\mathrm{CNe}$ in sample 1 , (d, e, f) for CNe in sample 2 .

whose distances calculated from two different methods (parallax and RDRs).

Recent studies of Kasliwal et al. (2011) and Shara et al. (2017) claimed that a new class of faint, fast and non-recurrent novae ( $t_{3}<20$ days) exists in M31 and M87. They adopted them as non-recurrent novae since the majority of them have low velocities (FWHM $<2500 \mathrm{~km} / \mathrm{s}$ ) and belong to Fe II class. These novae are up to 3 magnitudes fainter than predicted by the MMRD relation in Downes \& Duerbeck (2000). These studies caused a decrease in reliability of MMRD relations. This point should be further investigated to clarify the problems of the MMRD distributions, or to understand these types of novae. For example, Munari et al. (2017a) commented that none of the other surveys detected them during the whole observational history for M31 (e.g. Darnley et al. 2006). Moreover the OGLE microlensing survey has continuously monitored the Magellanic Clouds, where reddening is not an issue, no novae of the type claimed by Kasliwal et al. (2011) were detected (Mróz et al. 2016a). Munari et al. (2014; 2017a) suggested that there are systematic effects on Kasliwal et al. (2011)'s analyses owing to noisy and scarcely sampled light curves, frequently detecting the novae while already declining and thus probably missing the true maximum, and underestimated extinction estimates performed in an unusual way. Anyway, there is a possibility that this new type of novae exists in the Galaxy. For example, the novae in Table 1b (V977 Sco, V4332 Sgr, and MU Ser) were not added in the MMRD diagrams since their light curve parameters or distances/extinctions are not well-determined. If we assume their parameters are correct, their absolute magnitudes will be fainter than that calculated from our MMRD. If the distances/extinctions or light curve parameters are uncertain or biased, the possibility to verify the existence of an MMRD relation obviously decreases. Therefore, we only used the novae for which the distances and the interstellar extinctions were reliably determined. However, there is overall scattering in MMRD distributions similar (but larger) to that observed in Downes \& Duerbeck. Generally, this scattering is thought to be due to the distance and extinction, and Gaia DR2 or any other survey may minimize uncertainties on distance (or extinction) measurements that may decrease scattering on MMRD distributions.
However, the scattering would be caused by a second parameter (e.g. spectroscopic type, light-curve type, population type, mass of the underlying WD, chemical composition of the WD or of the accreted matter), which should influence the luminosity at outburst maximum. Just the WD mass, the accretion rate/envelope mass and the WD luminosity can produce a rich variety of nova outbursts. The heterogeneity in nova light curves suggests that a single parameter may not characterize the decline well. In our study, we suggest that the MMRD relation likely depends on other parameters in addition

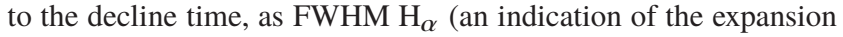
velocity), the light curve shapes (especially D- and J-types).

Analyses of the MMRD relation considering $\mathrm{FWHM} \mathrm{H}_{\alpha}$ show that the scattering on absolute magnitude is increasing with FWHM $\mathrm{H}_{\alpha}$, which is related to the expansion velocity. Moreover, for the $\mathrm{RN}+\mathrm{cRN}$ with medium velocities, we were able to obtain a relation, whose slope is larger. Recurrent novae (or the systems with high expansion velocity) seem to follow a different trend than that obtained for classical novae. From population models, Pagnotta \& Schaefer (2014) suggested that $25 \%$ of the systems labeled as $\mathrm{CN}$ are in fact RN for which only one outburst has thus far been discovered. However, we do not actually know the fraction between the classical novae and $\mathrm{RNe}$, and which nova systems are recurrent. There are only 10 known $\mathrm{RNe}$ on which the statistical studies depends. Considering the light curve shape, J-type novae have a constant absolute magnitude of -7 mag. The investigation on decline times for all novae in the catalogue indicates that the novae with D- and J- type light curve shapes may not have linear decline. If so, only considering the decline times $\left(t_{2}\right.$ or $\left.t_{3}\right)$ may not characterize the absolute magnitude at outburst maximum and the decline well. All of these arise uncertainties when using of MMRD relation if the type of nova is not clarified. Furthermore, the luminosities of novae may differ depending on their stellar populations or their evolutionary states; i.e. on the stellar age and metallicity. For the scatters found in other distance indicators, e.g. Cepheids, it has been suggested that a different metalicity of the host galaxy may significantly affect the zero-point of the relation (Freedman et al. 2001). In this case, there is a possibility on any environmental dependence on MMRD relations as well. Since our and the other studies in literature depend 
on disk novae in the Galaxy, the contribution of other populations (bulge, halo, even thick disk) is not well known. Our study does not dispel the concerns regarding the validity the MMRD relation, but has raised open questions. To understand the MMRD relation better, the novae in galaxies, which have reliable distances (and extinctions), should be studied with high quality photometric and spectroscopic data. A larger sample of them would also be very welcome as well as corresponding parameters and well-determined light curves that allow us to classify their light curve shapes. Our catalogue may also help to investigate the nature of MMRD diagrams in the long-term. Studying the MMRD relation is important to understand the origin of the nova eruption itself, besides using it as a tool to estimate absolute magnitudes.

In this study, we did not find any signs that the spectral classification represents the stellar population of the Galactic novae, as suggested by Della Valle \& Livio (1998). However, our decline time histogram implies that the $\mathrm{He} / \mathrm{N}+\mathrm{Hybrid}$ novae are fast systems with a peak time at $t_{3}=13$ days, while the slow Fe II novae have a peak decline time at $t_{3}=42$ days. In contrast to the suggestion of Della Valle \& Livio (1998), we found that both Fe II and $\mathrm{He} / \mathrm{N}$ novae or fast and slow novae are located in the whole range of vertical distances, but mainly concentrated throughout the Galactic disk. The differences between the results in these two studies may arise from the samples used in the analyses. The sample in Della Valle \& Livio (1998) contains a smaller number of novae with spectral classification, which reach only to a vertical distance of $\sim 2 \mathrm{kpc}$, whereas our sample have much more systems reaching up to two times farther in vertical direction from the Galactic plane. A relatively large number of slow novae located close to the Galactic plane and fast novae farther from Galactic plane must have been undetected in their analyses. The approach proposed by Della Valle \& Livio (1998) allowed us to classify the population of the novae in the host galaxy, however, it seems that kinematical and/or spectroscopic observations for the novae in different Galactic components are needed to clarify the population of the Galactic novae with certainty.

In order to understand the nova formation and evolution scenarios, key ingredients are Galactic model parameters such as space density and scale height (and scale length), which also describe the spatial distributions of these objects in the Galaxy. However, statistical and systematic uncertainties arising from the small number of systems and uncertain distances, affect the estimates of Galactic model parameters. In this study, Galactic model parameters of the Galactic novae were investigated with fairly homogeneous samples containing a large number of novae, but it is not free from the selection effect caused by undiscovered novae. However, limiting the volume occupied by novae makes the selection effects less significant. According to present samples and their analyses, the scale heights were calculated to be in the range of $0.15-0.20 \mathrm{kpc}$. It is important to conclude that exponential scale height of $0.16 \mathrm{kpc}$ for a mean value is best estimate for the Galactic novae. This is consistent with previous estimates, which depend on a small number of Galactic novae specifically; 0.150 kpc (Patterson 1984) 0.125 kpc (Duerbeck 1984), $0.164 \mathrm{kpc}$ (Özdönmez et al. 2015). However, these are smaller than the scale height of $0.25 \mathrm{kpc}$ estimated by Shafter (2017). For cataclysmic variables, the scale height estimates in the range of 0.1 - 0.2 kpc (Duerbeck 1984; Patterson 1984; van Paradijs et al. 1996; Ak et al. 2008; Revnivtsev et al. 2008; Özdönmez et al. 2015) have been made over the years, and our result is in this range, but it is smaller than the scale height of the thin disk population which lies in the range $0.2-0.3 \mathrm{kpc}$ (e.g. Cabrera-Lavers et al. 2005; Bilir et al. 2006; Jurić et al. 2008; Yaz Gökçe et al. 2015, and refer- ences therein). This indicates that the interactions in binary systems, and/or rapid outbursts may accelerate the evolutions of the novae in the Galaxy that cause them to lie closer to the Galactic plane. If so, it raises a question why a considerable number of outbursts at thick disk or halo can not be detected? A possible answer may be that the lifetimes of novae are much smaller than that of the Galactic (thin) disk, or that they change their sub-types of cataclysmic variables from (old) novae to dwarf novae or nova-like systems on relatively short time-scale, or that we just do not detect them due to observational effects.

For the outburst density, we used the Z-distributions of the classical novae within a cylinder of a radius $d_{X Y}=2 \mathrm{kpc}$, and calculated the outburst density as $3.6 \pm 0.25$ and $4.2 \pm 0.26 \times 10^{-10} \mathrm{pc}^{-3} \mathrm{yr}^{-1}$ for the novae in sample 1 and 2, respectively. The limits of the local outburst density were also calculated as 1.3 and $6 \times 10^{-10} \mathrm{pc}^{-3} \mathrm{yr}^{-1}$ by adopting cylinder radii of 1 and $4 \mathrm{kpc}$, respectively. This result represents very well the outburst density of novae in the solar neighbourhood compared to that obtained in previous studies (Patterson 1984; Duerbeck 1984; Naylor et al. 2009; della Valle \& Duerbeck 1993; Shafter 2017). The adopted outburst density allows us to infer a space density of classical novae in the range of $\sim 1-10 \times 10^{-6} \mathrm{pc}^{-3}$ when using $T_{R}=3000-23,000 \mathrm{yr}$, while the potential range of the space density was calculated as $0.4-16 \times 10^{-6} \mathrm{pc}^{-3}$. Since ratios between the recurrence times vary within an order of magnitude, these results are only limits for the actual space density. They are consistent with lower limit of $\sim 10^{-6}-10^{-8} \mathrm{pc}^{-3}$ in previous studies (Warner 1974; Patterson 1984; Duerbeck 1984; Downes 1986; della Valle \& Duerbeck 1993). The theoretical population analyses on cataclysmic variables predict a similar space density $10^{-5}-10^{-4}$ $\mathrm{pc}^{-3}$ (Ritter \& Burkert 1986; de Kool 1992; Kolb 1993; Politano 1996). However, two uncertainties should be taken into consideration in the calculation of the space density. First, the contribution of the $\mathrm{RNe}$ systems labeled as $\mathrm{CNe}$ effects the mean recurrence time and the space density. As we mentioned in the discussion on the MMRD relation, we do not know the actual fraction of $\mathrm{RNe}$ in the $\mathrm{CNe}$ samples. Even though, we excluded the RNe sample (including RN candidates proposed by Pagnotta \& Schaefer (2014)) from our calculations, a possible extra contribution may exists. Besides, we adopted a lower limit of 3000 years for the mean recurrence time of classical novae, and this does not represent recurrent novae whose recurrence time is in the range of 1 year (Darnley et al. 2016) up to $\sim 100$ years (Schaefer 2010). If we assume that all novae are actually recurrent novae, the space density can only be calculated by grouping novae with similar recurrence times, but this is not possible with the current Galactic novae sample. The other but unsolvable problem arises from an observational selection effect. The contribution from novae with unrecorded outbursts makes the space density results of the novae far from certain. Defining limits and using a larger sample in these analyses make the sample approximately complete in a certain volume. Nevertheless, selection effects on the sample in our study are likely less than previous samples in the literature. Thus, Galactic model parameters of the Galactic novae in the solar neighbourhood derived from this sample should be more reliable.

Using the local outburst density and scale height obtained in this study, we calculated the nova rate for the Galactic disk in the range of $\sim 20$ to $\sim 100$ per year using the same methodology as Shafter (2017). The average estimate of the disk nova rate for our samples is $67_{-17}^{+21} \mathrm{yr}^{-1}$, which is larger than the best estimate of $45 \mathrm{yr}^{-1}$ for disk nova rate obtained by Shafter (2017). Recently, Mróz et al. (2015) have measured a nova rate $13.8 \pm 2.6$ $\mathrm{yr}^{-1}$ for the Galactic bulge alone using OGLE observations. By adding this value, the global nova rate can be estimated as $\sim 80$ 
per year. In the literature, the Galactic nova rate is not well estimated and differs in the range between 20 and $260 y r^{-1}$ (Sharov 1972; della Valle \& Livio 1994; Shafter 1997, 2002, 2017), but our result is larger than typically adopted estimates for the Galaxy, and also larger than that calculated for the novae in other galaxies (Shafter et al. 2000; Darnley et al. 2006; Shafter et al. 2014). Since nova explosions contribute to the enrichment of the interstellar medium and to the chemical evolution of the Galaxy, the nova rate is essential for Galactic chemical evolution models. However, the incompleteness of a nova sample in the Galaxy gives rise to uncertainties on the measurements. The nova rate could be higher when considering the contributions of (undetected/old) novae corresponding to other populations.

\section{ACKNOWLEDGEMENTS}

We acknowledge the anonymous referee for helpful comments on this paper. We wish to thank Kai Schwenzer for his support on the proof reading. This work has been supported in part by the Scientific and Technological Research Council of Turkey (TÜBITAK) 114F271. TG was supported in part by the Scientific Research Project Coordination Unit of Istanbul University (Project no: 53496). This research has made use of the International Variable Star Index (VSX) database, operated at AAVSO, Cambridge, Massachusetts, USA. We acknowledge with thanks the variable star observations from the AAVSO International Database contributed by observers worldwide which were used in this research. This research has made use of the SIMBAD, and NASA's Astrophysics Data System Bibliographic Services.

\section{REFERENCES}

Ak T., Retter A., Liu A., 2005, PASA, 22, 298

Ak T., Bilir S., Ak S., Eker Z., 2008, NewA, 13, 133

Ak T., Bilir S., Özdönmez A., et al., 2015, Ap\&SS, 357, 72

Andrea J., Drechsel H., Starrfield S., 1994, A\&A, 291, 869

Andreae J., Drechsel H., Snijders M. A. J., Cassatella A., 1991, A\&A, 244, 111

Arai A., Nagashima M., Kajikawa T., Naka C., 2011, IAUC, 9203, 2

Arkhipova V. P., Burlak M. A., Esipov V. F., 2002, AstL, 28, 100

Ayani K., Kawabata Y., 2005, IAUC, 8501, 3

Ayani K., Murakami N., 2008, IAUC, 8948, 2

Ayani K., Kawabata T., Liller W., 2001, IAUC, 7589, 2

Ayani K., Murakami N., Hata K., et al., 2009, CBET, 1911, 1

Azaliah R., Malasan H. L., Haans G. K., Akhyar S., 2015, PKAS, 30, 251

Balman Ş., Retter A., Bos M., 2006, AJ, 131, 2628

Balman S., Nasiroglu I., Akyuz A., 2009, ATel, 2137,

Banerjee D. P. K., Srivastava M. K., Ashok N. M., Venkataraman V., 2016, MNRAS, 455, L109

Barbon R., Rosino L., Bond H., et al., 1989, IAUC, 4862, 1

Barsukova E. A., Valeev A. F., Goranskij V. P., Zharova A. V., 2013, ATel, 5454 ,

Belyakina T. S., Arkhipova V. P., Dokuchaeva O. D., Noskova R. I., 1988, IzKry, 78, 43

Bilir S., Karaali S., Ak S., Yaz E., Hamzaoğlu E., 2006, NewA, 12, 234

Binney J., Burnett B., Kordopatis G., et al., 2014, MNRAS, 437, 351

Birriel J. J., Espey B. R., Schulte-Ladbeck R. E., 2000, ApJ, 545, 1020

Bloch M., Dufay J., Fehrenbach C., Mao Lin T., 1946, AnAp, 9, 157

Bode M. F., Evans A., 2008, Classical Novae

Bode M. F., Harman D. J., O'Brien T. J., et al., 2007, ApJ, 665, L63

Brosch N., Leibowitz E. M., 1981, IAUC, 3596, 2

Brown N. J., Yamaoka H., West J. D., Liller W., 2003, IAUC, 8204, 1

Buil C., Fujii M., 2007, IAUC, 8862, 2
Burlak M. A., Henden A. A., 2008, AstL, 34, 241

Cabrera-Lavers A., Garzón F., Hammersley P. L., 2005, A\&A, 433, 173

Capaccioli M., della Valle M., D’Onofrio M., Rosino L., 1990, ApJ, 360, 63

Catchpole R. M., 1969, MNRAS, 142, 119

Cavagna M., Garradd G. J., Fujii M., et al., 1997, IAUC, 6678, 1

Chen H.-L., Woods T. E., Yungelson L. R., Gilfanov M., Han Z., 2016, MNRAS, 458, 2916

Chochol D., Grygar J., Pribulla T., et al., 1997, A\&A, 318, 908

Chochol D., Pribulla T., Katysheva N. A., Shugarov S. Y., Volkov I. M., 2005, Ap\&SS, 296, 135

Chochol D., Shugarov S., Pribulla T., Volkov I., 2014, CoSka, 43, 330

Chomiuk L., Linford J. D., Yang J., et al., 2014, Natur, 514, 339

Ciatti F., Rosino L., 1974, A\&AS, 16, 305

Cohen J. G., 1985, ApJ, 292, 90

Cohen J. G., Rosenthal A. J., 1983, ApJ, 268, 689

Cragg T., Charles P., Nikoloff I., et al., 1983, IAUC, 3766, 1

Csák B., Kiss L. L., Retter A., Jacob A., Kaspi S., 2005, A\&A, 429, 599

Czart K., Lewandowski M., Maciejewski G., et al., 2006, ATel, 792,

Darnley M. J., Bode M. F., Kerins E., et al., 2006, MNRAS, 369, 257

Darnley M. J., Ribeiro V. A. R. M., Bode M. F., Hounsell R. A., Williams R. P., 2012, ApJ, 746, 61

Darnley M. J., Henze M., Bode, M. F. et al., 2016, ApJ, 833, 149.

Das R., Banerjee D. P. K., Nandi A., Ashok N. M., Mondal S., 2015, MNRAS, 447, 806

de Kool M., 1992, A\&A, 261, 188

De Young J. A., Schmidt R. E., 1994, ApJ, 431, L47

della Valle M., 1993, IAUC, 5870, 1

della Valle M., Duerbeck H. W., 1993, A\&A, 275, 239

della Valle M., Livio M., 1994 A\&A, 286, 786

della Valle M., Livio M., 1995, ApJ, 452, 704

della Valle M., Benetti S., 1997, IAUC, 6532, 2

Della Valle M., Livio M., 1998, ApJ, 506, 818

della Valle M., Bianchini A., Livio M., Orio M., 1992a, A\&A, 266, 232.

della Valle M., Smette A., Dopita M. A., et al., 1992b, IAUC, 5529, 1

della Valle M., Cristiani S., Lissandrini C., Kilmartin P. M., Gilmore A. C., 1993, IAUC, 5863, 1

della Valle M., Mirabel I. F., Rodriguez L. F., 1994, A\&A, 290, 803

della Valle M., Pizzella A., Bernardi M., et al., 1998, IAUC, 6848, 1

Della Valle M., Pasquini L., Daou D., Williams R. E., 2002, A\&A, 390, 155

Diaz M. P., Steiner J. E., 1989, ApJ, 339, L41

Diaz M. P., Bruch A., 1997, A\&A, 322, 807

Dobrotka A., Retter A., Liu A., 2006, MNRAS, 371, 459

Dobrotka A., Retter A., Liu A., 2008, A\&A, 478, 815

Downes R. A., 1986, ApJ, 307, 170

Downes R. A., Duerbeck H. W., 2000, AJ, 120, 2007

Downes R. A., Webbink R. F., Shara M. M., et al., 2001, PASP, 113, 764

Duerbeck H. W., 1981, PASP, 93, 165

Duerbeck H. W., 1984, Ap\&SS, 99, 363

Duerbeck H. W., 1987, SSRv, 45, 1

Duerbeck H. W., Grebel E. K., 1993, MNRAS, 265, L9

Duerbeck H. W., Sterken C., Fu J. N., 2002, IAUC, 7974, 1

Ederoclite A., Mason E., Della Valle M., et al., 2006, A\&A, 459, 875

Eyres S. P. S., Davis R. J., Bode M. F., Lloyd H. M., 1996, IAUC, 6292, 2

Feast M. W., Lloyd Evans T., Mattei J. A., Snyder L. F., 1991, IAUC, 5233, 2

Finzell T., Chomiuk L., Munari U., Walter F. M., 2015, ApJ, 809, 160

Freedman W. L., Madore B. F., Gibson B. K., et al., 2001, ApJ, 553, 47.

Fujii M., 1998, IAUC, 7037, 2

Fujii M., Yamaoka H., 2005, IAUC, 8617, 3

Fujii M., Kato T., Takao A., Haseda K., Nakajima K., 2002, IAUC, 7882, 3

Gaia Collaboration, Prusti, T., de Bruijne, J. H. J., et al., 2016, A\&A, 595, A1

Gallagher J., Skiff B., Wagner R. M., et al., 1987, IAUC, 4501, 1

Gaposchkin C. H. P., 1957, The Galactic Novae.

Garnavich P., Howell E., Soderberg A., et al., 1999, IAUC, 7154, 2

Gill C. D., O’Brien T. J., 1998, MNRAS, 300, 221

Gill C. D., O'Brien T. J., 2000, MNRAS, 314, 175

Gilmozzi R., Selvelli P., 2007, A\&A, 461, 593 
Gilmozzi R., Selvelli P. L., Cassatella A., 1994, MmSAI, 65, 199

Goranskij V. P., Katysheva N. A., Kusakin A. V., et al., 2007, AstBu, 62, 125

Green G. M., Schlafly E. F., Finkbeiner D. P., et al., 2015, ApJ, 810, 25

Greiner J., Orio M., Schartel N., 2003, A\&A, 405, 703

Hachisu I., Kato M., 2007, ApJ, 662, 552

Hachisu I., Kato M., 2010, ApJ, 709, 680

Hachisu I., Kato M., 2014, ApJ, 785, 97

Hachisu I., Kato M., 2016a, ApJS, 223, 21

Hachisu I., Kato M., 2016b, ApJ, 816, 26

Hack W. J., Paresce F., 1993, PASP, 105, 1273

Hacke G., 1987, IBVS, 2979, 1

Harrison T. E., Bornak J., McArthur B. E., Benedict G. F., 2013, ApJ, 767, 7

Helton L. A., Woodward C. E., Vanlandingham K., Schwarz G. J., 2008a, IAUC, 8989, 2

Helton L. A., Woodward C. E., Vanlandingham K., Schwarz G. J., 2008b, CBET, 1448, 1

Helton L. A., Woodward C. E., Walter F. M., et al., 2010, AJ, 140, 1347

Helton L. A., Gehrz R. D., Woodward C. E., et al., 2012, ApJ, 755, 37

Henize K. G., McLaughlin D. B., 1951, AJ, 56, 74

Holdsworth D. L., Rushton M. T., Bewsher D., et al., 2014, MNRAS, 438, 3483

Hounsell R., Bode M. F., Hick P. P., et al., 2010a, ApJ, 724, 480

Hounsell R., Bode M. F., Hick P., et al., 2010b, ATel, 2558,

Hounsell R., Darnley M. J., Bode M. F., et al., 2011, A\&A, 530, A81

Iijima T., 2001, IAUC, 7666, 2

Iijima T., 2015, AJ, 150, 20

Iijima T., Rosino L., 1996, IAUC, 6365, 2

Iijima T., Esenoglu H. H., 2003, A\&A, 404, 997

Iijima T., Nakanishi H., 2008, A\&A, 482, 865

Iijima T., Correia A. P., Hornoch K., Carvajal J., 2007, CBET, 1006, 1

Jablonski F., Braga J., Gilmore A. C., 1991, IAUC, 5262, 2

Joshi V., Banerjee D. P. K., Ashok N. M., Venkataraman V., Walter F. M., 2015, MNRAS, 452, 3696

Jurić M., Ivezić Ž., Brooks A., et al., 2008, ApJ, 673, 864-914

Kasliwal M. M., Cenko S. B., Kulkarni S. R., et al., 2011, ApJ, 735, 94

Kato T., Fujii M., 2003, IAUC, 8132, 2

Kato T., Fujii M., Ayani K., 2002a, IAUC, 7975, 2

Kato T., Uemura M., Haseda K., et al., 2002b, PASJ, 54, 1009

Katysheva N. A., Shugarov S. Y., 2007, ASPC, 372, 523

Kingsburgh R. L., English J., Gilmore A. C., Bortle J. E., Scovil C., 1991, IAUC, 5320, 1

Kinugasa K., Honda S., Hashimoto O., Taguchi H., Takahashi H., 2009, IAUC, 9089, 2

Kiss L. L., Thomson J. R., 2000, A\&A, 355, L9

Kiss L. L., Thomson J. R., Ogloza W., Furész G., Sziládi K., 2001, A\&A, 366, 858

Kiss L. L., Gogh N., Vinkó J., et al., 2002, A\&A, 384, 982

Klare G., Krautter J., Wolf B., 1980, A\&A, 89, 282

Kolb U., 1993, A\&A, 271, 149

Korotkiy S., Sokolovsky K., Brown N. J., et al., 2012, CBET, 3089, 1

Kosai H., Maehara H., Huruhata M., et al., 1983, IAUC, 3782, 1

Krautter J., Vogt N., Klare G., et al., 1981, A\&A, 102, 337

Krautter J., Woodward C. E., Schuster M. T., et al., 2002, AJ, 124, 2888

Lane B. F., Retter A., Eisner J. A., et al., 2007, ApJ, 669, 1150

Liller W., 1993, IAUC, 5867, 2

Liller W., 2005, IAUC, 8559, 1

Liller W., Benetti S., Cappellaro E., et al., 1995, IAUC, 6130, 1

Liller W., Monard L. A. G., Africa S., Samus N. N., Kazarovets E., 2005a, IAUC, 8497, 1

Liller W., Jacques C., Pimentel E., Aguiar J. G. D. S., Shida R. Y., 2005b, IAUC, 8596, 1

Liller W., Yamaoka H., Yamamoto M., 2006, CBET, 405, 1

Linford J. D., Ribeiro V. A. R. M., Chomiuk L., et al., 2015, ApJ, 805, 136

Lipkin Y. M., Leibowitz E. M., 2008, MNRAS, 387, 289

Livingston C. M., Hearnshaw J. B., Gilmore A. C., Kilmartin P. M., 2001, IBVS, 5172, 1
Lloyd C., Guilbault P. R., 2002, IBVS, 5324, 1

Lyke J. E., Campbell R. D., 2009, AJ, 138, 1090

Lyke J. E., Koenig X. P., Barlow M. J., et al., 2003, AJ, 126, 993

Lynch D. K., Rudy R. J., Mazuk S., Puetter R. C., 2000, ApJ, 541, 791

Lynch D. K., Woodward C. E., Geballe T. R., et al., 2006a, ApJ, 638, 987

Lynch D. K., Russell R. W., Rudy R. J., Woodward C. E., 2006b, IAUC, 8706,2

Lucas, P. W., Hoare M. G., Longmore A., et al., 2008, MNRAS, 391, 136.

Malasan H. L., Suherli J., Wiyando E., et al., 2011, CBET, 2796, 4

Maoz D., Mannucci F., Nelemans G., 2014, ARA\&A, 52, 107

Mason E., Diaz M., Williams R. E., Preston G., Bensby T., 2010, A\&A, 516, A108

Mason E., Ederoclite A., Williams R. E., Della Valle M., Setiawan J., 2012, A\&A, 544, A149

Maxwell M. P., Rushton M. T., Darnley M. J., et al., 2012, MNRAS, 419, 1465

Mazuk S., Lynch D. K., Rudy R. J., et al., 2005, IAUC, 8644, 1

McNaught R. H., Lee S., Peterson B., Shanks T., 1987, IAUC, 4397, 1

Miroshnichenko A. S., 1988, SvA, 32, 298

Mróz P., Udalski A., Poleski R., et al., 2015, ApJS, 219, 26

Mróz P., Udalski A., Poleski R., et al., 2016a, ApJS, 222, 9

Mróz P., Udalski A., Pietrukowicz P., et al., 2016b, Natur, 537, 649

Munari U., 2014, ASPC, 490, 183

Munari U., Dallaporta S., 2010, IBVS, 5932, 1

Munari U., Goranskij V. P., Popova A. A., et al., 1996, A\&A, 315, 166

Munari U., Henden A., Vallenari A., et al., 2005, A\&A, 434, 1107

Munari U., Henden A., Pojmanski G., et al., 2006a, MNRAS, 369, 1755

Munari U., Siviero A., Navasardyan H., Dallaporta S., 2006b, A\&A, 452, 567

Munari U., Valisa P., Dalla Via G., Dallaporta S., 2007a, CBET, 852, 1

Munari U., Siviero A., Valisa P., et al., 2007b, CBET, 1183, 1

Munari U., Henden A., Valentini M., et al., 2008a, MNRAS, 387, 344

Munari U., Siviero A., Henden A., et al., 2008b, A\&A, 492, 145

Munari U., Dallaporta S., Ochner P., 2010a, IBVS, 5944, 1

Munari U., Henden A., Valisa P., Dallaporta S., Righetti G. L., 2010b, PASP, 122,898

Munari U., Joshi V. H., Ashok N. M., et al., 2011a, MNRAS, 410, L52

Munari U., Siviero A., Dallaporta S., et al., 2011b, NewA, 16, 209

Munari U., Ribeiro V. A. R. M., Bode M. F., Saguner T., 2011c, MNRAS, 410,525

Munari U., Dallaporta S., Castellani F., et al., 2013, MNRAS, 435, 771

Munari U., Ochner P., Dallaporta S., et al., 2014, MNRAS, 440, 3402

Munari U., Walter F. M., Henden A., et al., 2015a, IBVS, 6139, 1

Munari U., Henden A., Banerjee D. P. K., et al., 2015b, MNRAS, 447, 1661

Munari U., Dallaporta S., Cherini G., 2016, NewA, 47, 7

Munari U., Hambsch F.-J., Frigo A., 2017a, MNRAS, 469, 4341

Munari U., Hambsch F.-J., Frigo A., et al., 2017b, ATel, 1057,

Naik S., Banerjee D. P. K., Ashok N. M., 2009, MNRAS, 394, 1551

Naik S., Banerjee D. P. K., Ashok N. M., Das R. K., 2010, MNRAS, 404, 367

Naito H., Tokimasa N., 2007, IAUC, 8896, 2

Naito H., Fujii M., 2008, IAUC, 8972, 2

Naito H., Tokimasa N., Yamaoka H., Fujii M., 2005a, IAUC, 8576, 2

Naito H., Tokimasa N., Yamaoka H., 2005b, IAUC, 8484, 1

Naito H., Narusawa S., Yamaoka H., Fujita Y., 2007, IAUC, 8812, 2

Naito H., Mizoguchi S., Arai A., et al., 2012, A\&A, 543, A86

Nakano S., Nishimura H., Wakuda S., Kadota K., 2005, IAUC, 8483, 1

Nakano S., Tago A., Nishiyama K., Sakamoto T., 2007a, IAUC, 8821, 1

Nakano S., Nishimura H., Kadota K., et al., 2007b, IAUC, 8824, 1

Nakano S., Tago A., Itagaki K., Kadota K., 2007c, CBET, 1181, 1

Nakano S., Nishiyama K., Kabashima F., Kadota K., 2008a, IAUC, 8948, 3

Nakano S., Nishiyama K., Kabashima F., et al., 2008b, IAUC, 8950, 1

Nakano S., Yamaoka H., Itagaki K., et al., 2009, IAUC, 9064, 1

Naylor T., Charles P. A., Mukai K., Evans A., 1992, MNRAS, 258, 449

Ness J.-U., Schwarz G. J., Retter A., et al., 2007, ApJ, 663, 505

Ness J.-U., Starrfield S., Schwarz G. J., et al., 2008, IAUC, 8911, 2

Nishiyama K., Kabashima F., Pojmanski G., et al., 2009, IAUC, 9061, 1

Nishiyama K., Kabashima F., Sakurai Y., et al., 2010, IAUC, 9142, 1 
Nussbaumer H., Vogel M., 1990, A\&A, 236, 117

Ohshima O., Ayani K., Shimizu M., et al., 1995, IAUC, 6214, 1

Oksanen A., Harlingten C., Guido E., et al., 2011, IAUC, 9216, 3

Orio M., Balman S., della Valle M., Gallagher J., Oegelman H., 1996, ApJ, 466,410

Orio M., Covington J., Ögelman H., 2001, A\&A, 373, 542

Orio M., Tepedelenlioglu E., Starrfield S., Woodward C. E., Della Valle M., 2005, ApJ, 620, 938

Özdönmez A., Ak T., Bilir S., 2015, NewA, 34, 234

Özdönmez A., Güver T., Cabrera-Lavers A., Ak T., 2016, MNRAS, 461, 1177

Pagnotta A., Schaefer B. E., 2014, ApJ, 788, 164

Patterson J., 1984, ApJS, 54, 443

Pereira A. J. S., McGaha J. E., Young J., Rhoades H., 2007, IAUC, 8895, 1

Peters C. S., Thorstensen J. R., 2006, PASP, 118, 687

Pigulski A., 2009, IAUC, 9043, 2

Poggiani R., 2010, NewA, 15, 170

Pojmanski G., Yamaoka H., Bolt G., et al., 2004, IAUC, 8369, 1

Pojmanski G., Yamaoka H., Kiyota S., et al., 2006, IAUC, 8671, 1

Pojmanski G., Szczygiel D., Pilecki B., et al., 2009, IAUC, 9043, 1

Politano M., 1996, ApJ, 465, 338

Puetter R. C., Rudy R. J., Lynch D. K., et al., 2004, IAUC, 8370, 3

Ragan E., Brozek T., Suchomska K., et al., 2009, ATel, 2327,

Raj A., Ashok N. M., Banerjee D. P. K., 2011, MNRAS, 415, 3455

Raj A., Ashok N. M., Banerjee D. P. K., et al., 2012, MNRAS, 425, 2576

Raj A., Banerjee D. P. K., Ashok N. M., 2013, MNRAS, 433, 2657

Raj A., Ashok N. M., Rudy R. J., et al., 2015a, AJ, 149, 136

Raj A., Banerjee D. P. K., Ashok N. M., Kim S. C., 2015b, RAA, 15, 993

Read A. M., Saxton R. D., Torres M. A. P., et al., 2008, A\&A, 482, L1

Revnivtsev M., Sazonov S., Krivonos R., Ritter H., Sunyaev R., 2008, A\&A, 489,1121

Richtler T., Liller W., 1986, IBVS, 2871, 1

Ringwald F. A., Naylor T., 1997, A\&A, 326, 629

Ritter H., Burkert A., 1986, A\&A, 158, 161

Rodríguez-Gil P., Santander-García M., Knigge C., et al., 2010, MNRAS, 407, L21

Ruch G., Polomski E., Woodward C. E., et al., 2003, IAUC, 8204, 2

Rudy R. J., Meier S. R., Rossano G. S., et al., 1999, ApJS, 121, 533

Rudy R. J., Russell R. W., Lynch D. K., Widths I., 2005, IAUC, 8523, 4

Rudy R. J., Lynch D. K., Russell R. W., Woodward C. E., 2007, IAUC, 8884, 2

Rudy R. J., Lynch D. K., Russell R. W., Gilbert A. M., Woodward C. E., 2008, IAUC, 8957, 1

Rudy R. J., Russell R. W., Sitko M., 2011, IAUC, 9211, 2

Rudy R. J., Crawford K. B., Russell R. W., 2016, ATel, 9849,

Rushton M. T., Evans A., Eyres S. P. S., van Loon J. T., Smalley B., 2008, MNRAS, 386, 289

Russell R. W., Rudy R. J., Lynch D. K., Golisch W., 2005a, IAUC, 8524, 2

Russell R. W., Lynch D. K., Rudy R. J., 2005b, IAUC, 8527, 1

Russell R. W., Rudy R. J., Lynch D. K., Woodward C. E., 2006, IAUC, 8710, 2

Russell R. W., Rudy R. J., Lynch D. K., et al., 2007, IAUC, 8846, 1

Russell R. W., Rudy R. J., Lynch D. K., Gilbert A. M., Woodward C. E., 2008, IAUC, 8956, 1

Saito R. K, Hempel M., Minniti D., et al., 2012, A\&A, 537A, 107.

Sanford R. F., 1940, PASP, 52, 35

Schaefer B. E., 2009, ApJ, 697, 721

Schaefer B. E., 2010, ApJS, 187, 275

Schaefer B. E., Landolt A. U., Linnolt M., et al., 2013, ApJ, 773, 55

Schmidtobreick L., Tappert C., Bianchini A., Mennickent R. E., 2003, A\&A, 410, 943

Schwarz G. J., Osborne J. P., Page K. L., et al., 2009, ATel, 2157,

Schwarz G. J., Ness J.-U., Osborne J. P., et al., 2011, ApJS, 197, 31

Sekiguchi K., Feast M. W., Whitelock P. A., et al., 1988, MNRAS, 234, 281

Sekiguchi K., Feast M. W., Fairall A. P., Winkler H., 1989, MNRAS, 241, 311

Selvelli P., 2004, BaltA, 13, 93

Selvelli P., Gilmozzi R., 2013, A\&A, 560, A49
Senziani F., Skinner G. K., Jean P., Hernanz M., 2008, A\&A, 485, 223

Shafter A. W., 1997, ApJ, 487, 226

Shafter A. W., 2002, AIPC, 637, 462

Shafter A. W., 2017, ApJ, 834, 196

Shafter A. W., Ciardullo R., Pritchet C. J., 2000, ApJ, 530, 193

Shafter A. W., Rau A., Quimby R. M., et al., 2009, ApJ, 690, 1148

Shafter A. W., Curtin C., Pritchet C. J., Bode M. F., Darnley M. J., 2014, ASPC, 490, 77

Shara M. M., Doyle T., Lauer T. R., et al., 2017, ApJ, 839, 109

Sharma S., Bland-Hawthorn J., Johnston K. V., Binney J., 2011, ApJ, 730,

Sharov A. S., 1972, SvA, 16, 41

Shears J., Poyner G., 2007, JBAA, 117, 136

Sheffer Y., Krisciunas K., 1987, IAUC, 4293, 2

Siviero A., Munari U., Jones A. F., 2005, IBVS, 5638, 1

Skrutskie M. F., Cutri, R. M., Stiening, R., et al., 2006, AJ, 131, 1163.

Slavin A. J., O'Brien T. J., Dunlop J. S., 1995, MNRAS, 276, 353

Slovak M. H., Vogt S. S., 1979, Natur, 277, 114

Snijders M. A. J., 1987, Ap\&SS, 130, 243

Srivastava M. K., Ashok N. M., Banerjee D. P. K., Sand D., 2015, MNRAS, 454, 1297

Srivastava M., Joshi V., Banerjee D. P. K., Ashok N. M., 2016, ATel, 8809,

Starrfield S., Sparks W. M., Truran J. W., 1985, ApJ, 291, 136

Starrfield S., Iliadis C., Hix W. R., Timmes F. X., Sparks W. M., 2009, ApJ, 692,1532

Strömgren B., Shapley H., 1951, HarAC, 1119,

Stringfellow G. S., Gregg M. D., McNaught R. H., et al., 1992, IAUC, 5635, 1

Strope R. J., Schaefer B. E., Henden A. A., 2010, AJ, 140, 34

Swierczynski E., Ragan E., Galan C., Mikolajewski M., 2008, ATel, 1723,

Szkody P., Ingram D., 1994, ApJ, 420, 830

Tajitsu A., Sadakane K., Naito H., Arai A., Aoki W., 2015, Natur, 518, 381

Tappert C., Schmidtobreick L., Vogt N., Ederoclite A., 2013, MNRAS, 436, 2412

Tappert C., Vogt N., Della Valle M., Schmidtobreick L., Ederoclite A., 2014, MNRAS, 442, 565

Tappert C., Vogt N., Schmidtobreick L., Ederoclite A., 2015, MNRAS, 450, 943

Tappert C., Barria D., Fuentes Morales I., et al., 2016, MNRAS, 462, 1371

Tarasova T. N., 2015, ARep, 59, 920

Thorstensen J. R., Taylor C. J., 2000, MNRAS, 312, 629

Tomov T., Moro D., Munari U., 1999, IAUC, 7225, 1

Tomov T., Mikolajewski M., Ragan E., Swierczynski E., Wychudzki P., 2008, ATel, 1475,

Tomov T., Swierczynski E., Mikolajewski M., Ilkiewicz K., 2015, A\&A, 576, A119

van Paradijs J., Augusteijn T., Stehle R., 1996, A\&A, 312, 93

Vanlandingham K. M., Starrfield S., Wagner R. M., Shore S. N., Sonneborn G., 1996, MNRAS, 282, 563

Vanlandingham K., Wagner R. M., Starrfield S. G., 2001, IAUC, 7696, 3

Vanlandingham K. M., Schwarz G., Starrfield S., et al., 2007, AAS, 39, 04.02

Venturini C. C., Rudy R. J., Lynch D. K., Mazuk S., Puetter R. C., 2004, AJ, 128, 405

Vrba F. J., Schmidt G. D., Burke E. W., Jr., 1977, ApJ, 211, 480

Waagen E. O., 2012, AAN, 455,

Waagen E. O., Henden A., Maury A., et al., 2008, IAUC, 8999, 1

Wagner R. M., Bertram R., Starrfield S. G., 1990, IAUC, 5006, 1

Wagner R. M., Starrfield S. G., Gilmore A. C., 1991, IAUC, 5243, 1

Wagner R. M., Vandlandingham K. M., King N., et al., 1994, IAUC, 6002 , 1

Wagner R. M., Starrfield S. G., Rohrbach J., et al., 2003, IAUC, 8176, 3

Wagner R. M., Dong S., Bensby T., et al., 2012, CBET, 3136, 1

Walter F. M., 2011, CBET, 2796, 3

Walter F. M., 2015, ASPC, 493, 507

Walter F. M., Battisti A., Towers S. E., Bond H. E., Stringfellow G. S., 2012, PASP, 124, 1057

Warner B., 1974, MNSSA, 33, 21 


\section{6 Özdönmez, A. et al.}

Warner B., 1987, MNRAS, 227, 23

Warner B., 1995, Cataclysmic Variable Stars (Cambridge: Cambridge University Press)

Warner B., Woudt P. A., 2009, MNRAS, 397, 979

Webbink R. F., Livio M., Truran J. W., Orio M., 1987, ApJ, 314, 653

Weight A., Evans A., Albinson J. S., Krautter J., 1993, A\&A, 268, 294

Weight A., Evans A., Naylor T., Wood J. H., Bode M. F., 1994, MNRAS, 266, 761

Weller W. G., Heathcote S. R., Seargent D. A. J., McNaught R. H., Pereira A., 1988, IAUC, 4594, 1

Wesson R., Barlow M. J., Corradi R. L. M., et al., 2008, ApJ, 688, L21

Weston J. H. S., Sokoloski J. L., Chomiuk L., et al., 2016, MNRAS, 460, 2687

Whitelock P. A., Carter B. S., Feast M. W., et al., 1984, MNRAS, 211, 421

Whitney B. A., Clayton G. C., 1989, AJ, 98, 297

Williams R. E., 1992, AJ, 104, 725

Williams R. E., 1994, ApJ, 426, 279

Williams R., 2012, AJ, 144, 98

Williams R., 2013, AJ, 146, 55

Williams S. C., Darnley M. J., 2016, ATel, 9628,

Williams S. C., Darnley M. J., 2017, ATel, 1054,

Williams R. E., Hamuy M., Phillips M. M., et al., 1991, ApJ, 376, 721

Williams R. E., Phillips M. M., Hamuy M., 1994, ApJS, 90, 297

Williams P., Waagen E. O., Krajci T., Schmeer P., 2006, IAUC, 8700, 1

Williams, R., Mason E., Della Valle M., Ederoclite A., 2008, ApJ, 685, 451

Woudt P. A., Warner B., 2001, MNRAS, 328, 159

Woudt P. A., Warner B., 2003, MNRAS, 343, 313

Woudt P. A., Ribeiro V. A. R. M., 2014, ASPC, 490,

Woudt P. A., Steeghs D., Karovska M., et al., 2009, ApJ, 706, 738

Yamaoka H., Fujii M., 2005, IAUC, 8506, 3

Yamaoka H., Waagen E., Amorim A., et al., 2007a, IAUC, 8807, 1

Yamaoka H., Nakamura Y., Itagaki K., Nakano S., Nishimura H., 2007b, IAUC, 8810,1

Yamaoka H., Itagaki K., Guido E., et al., 2009, IAUC, 9098, 1

Yan Tse J., Hearnshaw J. B., Rosenzweig P., et al., 2001, MNRAS, 324, 553

Yaron O., Prialnik D., Shara M. M., Kovetz A., 2005, ApJ, 623, 398

Yaz Gökçe E., Karaali S., Duran Ş., et al., 2015, PASA, 32, e012

Young P. R., Berrington K. A., Lobel A., 2005, A\&A, 432, 665

Zhao P., McClintock J. E., 1997, ApJ, 483, 899

Zwitter T., Munari U., 1996, A\&AS, 117, 449 
Table 5. The Galactic novae whose distances are known. We list Galactic coordinates $l$ and $b$, the amplitude of outburst, the visual maximum magnitude at outburst $V_{m a x}$, the decline times $t_{2,3}$ as light curve parameters. Type of novae corresponding to their light curve shape (LC; Strope et al. 2010), the spectral type and recurrence type are given. The full width at half maximum (FWHM) is measured from the Balme lines at or near outburst maximum. $P_{\text {or }}$ is the orbital period of the nova. The adopted distance $(D)$ calculations are given with their measurement methods. $E(B-V)$ gives the adopted reddening estimates obtained from the literature. Numbers in parenthesis are the reference codes.

\begin{tabular}{|c|c|c|c|c|c|c|c|c|c|c|c|c|c|c|}
\hline Nova & $\begin{array}{c}l \\
(\mathrm{deg})\end{array}$ & $\begin{array}{c}b \\
\text { (deg) }\end{array}$ & $\underset{(\text { mag) }}{\text { Amplitude }^{a}}$ & $\begin{array}{l}V_{\max } \\
(\mathrm{mag})\end{array}$ & $\begin{array}{c}t_{2} \\
\text { (days) }\end{array}$ & $\begin{array}{c}t_{3} \\
\text { (days) }\end{array}$ & LC type & Spec type & RN type & $\begin{array}{c}\text { FWHM } \\
(\mathrm{km} / \mathrm{s})\end{array}$ & $\begin{array}{l}P_{\text {orb }} \\
\text { (days) }\end{array}$ & $\begin{array}{c}D \\
(\mathrm{kpc})\end{array}$ & Method $^{b}$ & $\begin{array}{c}E(B-V) \\
(\mathrm{mag})\end{array}$ \\
\hline CI Aql & 31.688 & -0.812 & 7.7 (V) (1) & $9(1)$ & $25(1)$ & $32(1)$ & $\mathrm{P}(1)$ & & RN (2) & $2250(3)$ & $0.618(4)$ & $3.9 \pm 0.5$ & rdr (5) & $0.92 \pm 0.22(6)$ \\
\hline V500 Aql & 47.608 & -9.462 & $11.2(\mathrm{p})(4)$ & $6.1(7)$ & 17 (7) & $42(7)$ & & $\mathrm{He} / \mathbf{N}$ ? (7) & & $1400(8)$ & $0.145(4)$ & $5.9 \pm 1.2$ & epar (9) & $0.16 \pm 0.03(10)$ \\
\hline V603 Aql & 33.164 & 0.829 & $12.2(\mathrm{~V})(1)$ & $-0.5(1)$ & $5(1)$ & $12(1)$ & $\mathrm{O}(1)$ & hybrid (1) & & & $0.138(11)$ & $0.34 \pm 0.06$ & $\operatorname{tpar}(12)$ & $0.08 \pm 0.02(13)$ \\
\hline V1229 Aq1 & 40.537 & -5.437 & 11.5 (V) (1) & $6.6(1)$ & $18(1)$ & $32(1)$ & $\mathrm{P}(1)$ & Fe II (7) & cRN: (14) & & & $2.4 \pm 0.6$ & epar (9) & $0.5 \pm 0.08(15)$ \\
\hline V1494 Aql & 40.974 & -4.742 & $13(\mathrm{~V})(1)$ & $4.1(1)$ & $8(1)$ & $16(1)$ & $\mathrm{O}(1)$ & Fe II (16) & & $2470(17)$ & 0.135 (4) & $1.2 \pm 0.2$ & epar (18) & $0.6 \pm 0.1(19)$ \\
\hline V1663 Aql & 39.161 & -0.665 & & $11.2(20)$ & $16(20)$ & $34(20)$ & & Fe II (21) & & $1900(22)$ & & $8.9 \pm 3.6$ & epar (23) & $1.1(23)$ \\
\hline $\mathrm{V} 1721 \mathrm{Aql}^{d}$ & 40.972 & -0.083 & $<6$ (c) (14) & $14(24)$ & $6(24)$ & $10(24)$ & & & cRN (14) & 6450 (24) & & $7.5 \pm 2.0$ & $\operatorname{rdr}(6)$ & $3.0 \pm 0.6(25)$ \\
\hline $\mathrm{OY} \mathrm{Ara}^{d}$ & 333.903 & -3.937 & 11.5 (p) (4) & $5.1(26)$ & 44 (27) & $80(26)$ & & & & & 0.155 (4) & $1.32 \pm 0.7$ & $\operatorname{rdr}(6)$ & $0.32 \pm 0.06(28)$ \\
\hline T Aur & 177.143 & -1.699 & 10.4 (V) (1) & $4.5(1)$ & $80(1)$ & $84(1)$ & $\mathrm{D}(1)$ & Fe II (7) & & & 0.204 (4) & $0.96 \pm 0.22$ & epar (29) & $0.42 \pm 0.08(13)$ \\
\hline V705 Cas & 113.66 & -4.096 & 10.7 (V) (1) & $5.7(1)$ & $33(1)$ & $67(1)$ & $\mathrm{D}(1)$ & Fe II (21) & & & $0.228(30)$ & $2.5 \pm 0.5$ & epar (31) & $0.41 \pm 0.06(6)$ \\
\hline V723 Cas & 124.961 & -8.807 & 8.6 (V) (1) & $7.1(1)$ & $263(1)$ & $299(1)$ & $\mathrm{J}(1)$ & Fe II (32) & & $600(33)$ & $0.69327(32)$ & $3.86 \pm 0.23$ & epar (34) & $0.45(35)$ \\
\hline V842 Cen & 316.574 & 2.453 & 10.9 (V) (1) & $4.9(1)$ & $43(1)$ & $48(1)$ & $\mathrm{D}(1)$ & Fe II (1) & & $760(36)$ & $0.164(37)$ & $1.2 \pm 0.1$ & epar (7) & $0.55 \pm 0.05(6)$ \\
\hline V1065 Cen & 293.984 & 3.613 & $<9.8$ (V) (37) & $7.6(38)$ & $11(38)$ & $26(38)$ & & Fe II (38) & & $2700(38)$ & & $3.3 \pm 0.5$ & $\operatorname{rdr}(6)$ & $0.47 \pm 0.05(6)$ \\
\hline T CrB & 42.374 & 48.165 & $7.3(\mathrm{~V})(1)$ & $2.5(1)$ & $4(1)$ & $6(1)$ & $\mathrm{S}(1)$ & & RN (2) & $4980(39)$ & $227.55(40)$ & $0.9 \pm 0.2$ & comp. (2) & $0.1 \pm 0.08(2)$ \\
\hline CP Cru & 297.872 & 2.223 & 10.2 (V) (4) & $9.25(41)$ & $4(41)$ & $10(41)$ & & & cRN (14) & $2000(42)$ & $0.944(4)$ & $2.6 \pm 0.5$ & epar (41) & $1.9 \pm 0.1(41)$ \\
\hline V407 Cyg & 86.983 & -0.482 & 9 (V) (37) & $7.1(43)$ & $5.9(43)$ & $24(43)$ & & $\mathrm{He} / \mathrm{N}(43)$ & cRN (44) & 2300 (44) & $766.4(37)$ & $3.5 \pm 0.3$ & rdr (6) & $0.55 \pm 0.05(6)$ \\
\hline V450 Cyg & 79.129 & -6.458 & $8.5(\mathrm{p})(4)$ & $7.5(7)$ & $88(7)$ & $108(7)$ & & Fe II (7) & & & & $3.5 \pm 0.8$ & epar (29) & $0.33 \pm 0.03(10)$ \\
\hline V476 Cyg & 87.368 & 12.418 & 14.3 (V) (1) & $1.9(1)$ & $6(1)$ & $16(1)$ & $\mathrm{D}(1)$ & $\mathrm{Fe}$ II (7) & & & & $1.62 \pm 0.12$ & epar (29) & $0.18 \pm 0.1(45)$ \\
\hline V1500 Cyg & 89.823 & -0.072 & 16 (V) (1) & $1.9(1)$ & $2(1)$ & $4(1)$ & $S(1)$ & hybrid (16) & & & $0.14(4)$ & $1.5 \pm 0.2$ & epar (29) & $0.45 \pm 0.07(6)$ \\
\hline $\begin{array}{l}\text { V1819 Cyg } \\
\text {. }\end{array}$ & 71.372 & 3.978 & 7.7 (V) (1) & $9.3(1)$ & $95(1)$ & $181(1)$ & $\mathrm{J}(1)$ & Fe II (7) & & $2742(46)$ & & $7.4 \pm 1.4$ & epar (7) & $0.35 \pm 0.15(46)$ \\
\hline V1974 Cyg & 89.134 & 7.819 & $12.6(\mathrm{~V})(1)$ & $4.3(1)$ & 19 (1) & $43(1)$ & $\mathrm{P}(1)$ & Fe II (16) & & $2000(47)$ & $0.08126(48)$ & $3.0 \pm 0.2$ & epar (16) & $0.26 \pm 0.03(49)$ \\
\hline V2274 Cyg & 73.042 & 1.991 & $>8.5$ (V) (1) & $11.5(1)$ & $22(1)$ & $33(1)$ & $\mathrm{D}(1)$ & & & $950(50)$ & & $9.0 \pm 2.0$ & $\operatorname{rdr}(6)$ & $1.33 \pm 0.1(6)$ \\
\hline V2275 Cyg & 89.317 & 1.391 & 11.5 (V) (1) & $6.9(1)$ & $3(1)$ & $8(1)$ & $\mathrm{S}(1)$ & & cRN: (14) & $2100(51)$ & $0.315(4)$ & $3.62 \pm 0.47$ & $\operatorname{rdr}(6)$ & $1.0 \pm 0.1(51)$ \\
\hline $\begin{array}{l}\text { V2467 Cyg } \\
\text { V }\end{array}$ & 80.069 & 1.842 & $>11.6(\mathrm{~V})(1)$ & $7.4(1)$ & $8(1)$ & $20(1)$ & $\mathrm{O}(1)$ & Fe II & & $950(52)$ & $0.159(53)$ & $1.5 \pm 0.3$ & rdr (6) & $1.4 \pm 0.2(6)$ \\
\hline V2468 Cyg & 66.808 & 0.246 & & 7.57 (1) & $9(1)$ & $20(1)$ & & hybrid (54) & & $1800-2300(54)$ & $0.242(55)$ & $6.8 \pm 1.0$ & $\operatorname{rdr}(6)$ & $0.78 \pm 0.1(6)$ \\
\hline V2491 Cyg & 67.229 & 4.353 & $\sim 12.5(\mathrm{~V})(1)$ & $7.5(1)$ & $4(1)$ & $16(1)$ & C (1) & $\mathrm{He} / \mathrm{N}(56)$ & cRN (14) & $4860(57)$ & & $2.1 \pm 1.4$ & rdr (6) & $0.23 \pm 0.01(58)$ \\
\hline HR Del & 63.43 & -13.972 & 8.5 (V) (1) & $3.6(1)$ & 167 (1) & $231(1)$ & $\mathrm{J}(1)$ & Fe II (7) & & & $0.214(4)$ & $1.01 \pm 0.62$ & $\operatorname{tpar}(12)$ & $0.17 \pm 0.02(13)$ \\
\hline DQ Her & 73.153 & 26.444 & 12.7 (V) (1) & $1.6(1)$ & $76(1)$ & $100(1)$ & $\mathrm{D}(1)$ & Fe II (7) & & & 0.194 (4) & $0.39 \pm 0.03$ & tpar (59) & $0.05 \pm 0.02(13)$ \\
\hline V446 Her & 45.409 & 4.708 & 11.3 (V) (1) & $4.8(1)$ & $20(1)$ & $42(1)$ & $\mathrm{S}(1)$ & $\mathrm{He} / \mathrm{N}(1)$ & & & $0.207(60)$ & $1.35 \pm 0.26$ & epar (9) & $0.37 \pm 0.04(6)$ \\
\hline V533 Her & 69.189 & 24.273 & 12 (V) (1) & $3(1)$ & $30(1)$ & $43(1)$ & $\mathrm{S}(1)$ & Fe II (1) & & & $0.21(30)$ & $1.25 \pm 0.3$ & epar (61) & $0.03 \pm 0.02(13)$ \\
\hline CP Lac & 102.141 & -0.837 & 13 (V) (1) & $2(1)$ & $5(1)$ & $9(1)$ & $\mathrm{s}(1)$ & hybrid (7) & & & $0.145(11)$ & $1.35 \pm 0.26$ & epar (62) & $0.27 \pm 0.06(6)$ \\
\hline DK Lac & 105.237 & -5.352 & 7.9 (V) (1) & $5.9(1)$ & $55(1)$ & $202(1)$ & $J(1)$ & Fe II (7) & & & $0.1296(63)$ & $3.9 \pm 0.5$ & epar (29) & $0.22 \pm 0.06(13)$ \\
\hline BT Mon & 213.859 & -2.623 & 7.6 (V) (1) & $8.1(1)$ & 118 (1) & $182(1)$ & $\mathrm{F}(1)$ & & cRN: (14) & $2100(64)$ & 0.334 (4) & $1.8 \pm 0.3$ & epar (65) & $0.24 \pm 0.06(13)$ \\
\hline IM Nor & 327.098 & 2.485 & 9.8 (V) (1) & $8.5(1)$ & $50(1)$ & $80(1)$ & $\mathrm{P}(1)$ & & $\mathrm{RN}(2)$ & $2380(66)$ & $0.102(67)$ & $2.4 \pm 0.8$ & rdr (6) & $0.8 \pm 0.2(6)$ \\
\hline V382 Nor & 332.29 & -0.995 & $<10.5(\mathrm{~V})(37)$ & $8.9(68)$ & $12(69)$ & $18(69)$ & & Fe II (21) & & $1850(22)$ & & $4.0 \pm 0.53$ & rdr (6) & $0.85 \pm 0.15(6)$ \\
\hline RS Oph & 19.8 & 10.372 & $6.2(\mathrm{~V})(1)$ & $4.8(1)$ & $7(1)$ & $14(1)$ & $\mathrm{P}(1)$ & & RN (2) & $3930(70)$ & $455.72(4)$ & $4.2 \pm 0.9$ & comp. (2) & $0.73 \pm 0.1(71)$ \\
\hline V2487 Oph & 6.604 & 7.775 & 8.2 (V) (1) & $9.5(1)$ & $6(1)$ & $8(1)$ & $\mathrm{P}(1)$ & & $\mathrm{RN}(2)$ & $10000(72)$ & & $1.12 \pm 0.33$ & $\operatorname{rdr}(6)$ & $0.4 \pm 0.08(6)$ \\
\hline V2615 Oph & 4.148 & 3.302 & $<11.4$ (V) (37) & $8.52(73)$ & $26.5(73)$ & $48.5(73)$ & & Fe II (74) & & $800(75)$ & & $2.1 \pm 0.8$ & $\operatorname{rdr}(6)$ & $0.95 \pm 0.15(6)$ \\
\hline V2672 Oph & 1.019 & 2.529 & $<10.3(\mathrm{~V}, \mathrm{c})(37)$ & $10(76)$ & $2.3(77)$ & $6(14)$ & & $\mathrm{He} / \mathrm{N}(74)$ & cRN (14) & $8000(78)$ & & $3.12 \pm 0.69$ & $\operatorname{rdr}(6)$ & $1.6 \pm 0.1(43)$ \\
\hline GK Per & 150.956 & -10.104 & 12.8 (V) (1) & $0.2(1)$ & $6(1)$ & $13(1)$ & $\mathrm{O}(1)$ & Fe II (7) & & & $1.9968(4)$ & $0.48 \pm 0.03$ & tpar (59) & $0.34 \pm 0.04(13)$ \\
\hline RR Pic & 272.355 & -25.672 & 11.2 (V) (1) & $1(1)$ & $73(1)$ & $122(1)$ & $J(1)$ & Fe II (7) & & & $0.145(4)$ & $0.41 \pm 0.07$ & tpar (12) & $0.0 \pm 0.02(13)$ \\
\hline CP Pup & 252.926 & -0.835 & $>18.8$ (V) (1) & $0.7(1)$ & $4(1)$ & $8(1)$ & $\mathrm{P}$ & $\mathrm{He} / \mathrm{N}(7)$ & cRN: (14) & & 0.061 (4) & $1.14 \pm 0.23$ & epar (7) & $0.2(79)$ \\
\hline V351 Pup & 252.723 & -0.733 & 13.2 (V) (1) & $6.4(1)$ & $9(1)$ & $26(1)$ & $\mathrm{P}(1)$ & Fe II (21) & & & $0.1182(80)$ & $4.7 \pm 0.6$ & epar (81) & $0.48 \pm 0.1(22)$ \\
\hline V445 Pup & 241.124 & -2.192 & $6(\mathrm{~V})(1)$ & $8.6(1)$ & $215(1)$ & 240 (1) & $\mathrm{D}(1)$ & Fe II ( 82$)$ & & & $0.65065(37)$ & $8.2 \pm 0.3$ & epar (83) & $0.51(82)$ \\
\hline T Pyx & 257.207 & 9.707 & $9.1(\mathrm{~V})(1)$ & $6.4(1$ & $32(1)$ & $62(1)$ & $\mathrm{P}(1)$ & hybrid (84) & $\mathrm{RN}(2)$ & $5350(85)$ & $0.076(4)$ & $4.8 \pm 0.5$ & mult. (86) & $0.25 \pm 0.02(87)$ \\
\hline U Sco & 357.669 & 21.869 & 10.1 (V) (1) & $7.5(1)$ & $1(1)$ & $3(1)$ & $\mathrm{P}(1)$ & $\mathrm{He} / \mathrm{N}(88)$ & $\mathrm{RN}(2)$ & $5700(89)$ & 1.231 (4) & $12.0 \pm 2.0$ & comp. (2) & $0.14 \pm 0.12(90)$ \\
\hline V745 Sco & 357.358 & -3.999 & $9.2(2)$ & $9.4(2)$ & $6.2(2)$ & $9(2)$ & & $\mathrm{He} / \mathrm{N}(21)$ & $\mathrm{RN}(2)$ & $3600(91)$ & $510.0(92)$ & $: 7.8 \pm 1.8$ & comp. (2) & $1.0 \pm 0.2(2)$ \\
\hline V1187 Sco & 355.724 & 1.451 & $8.2(\mathrm{~V})(1)$ & $9.8(1)$ & $10(1)$ & $17(1)$ & $\mathrm{S}(1)$ & Fe II (21) & cRN: (14) & $3000(93)$ & $354.0(37)$ & $3.2 \pm 0.12$ & $\operatorname{rdr}(6)$ & $1.3(93)$ \\
\hline V1280 Sco & 351.331 & 6.553 & 17.21 (V) (94) & $3.79(95)$ & $21.3(94)$ & $34.3(94)$ & & Fe II (74) & & $640(96)$ & & $1.1 \pm 0.5$ & epar (97) & $0.32 \pm 0.05(6)$ \\
\hline V1309 Sco & 359.786 & -3.135 & 12.5 (V) (37) & $7.9(98)$ & $20(98)$ & $25(98)$ & & & & $670(99)$ & & $2.5 \pm 0.4$ & rdr (6) & $0.55 \pm 0.05(6)$ \\
\hline
\end{tabular}


Table 5 - continued

\begin{tabular}{|c|c|c|c|c|c|c|c|c|c|c|c|c|c|c|}
\hline Nova & $\begin{array}{c}l \\
(\mathrm{deg})\end{array}$ & $\begin{array}{c}b \\
(\mathrm{deg})\end{array}$ & $\underset{\text { (mag) }}{\text { Amplitude }^{a}}$ & $\begin{array}{r}V_{\max } \\
(\operatorname{mag})\end{array}$ & $\begin{array}{c}t_{2} \\
\text { (days) }\end{array}$ & $\begin{array}{c}t_{3} \\
\text { (days) }\end{array}$ & LC type & Spec type & RN type & $\begin{array}{c}\mathrm{FWHM} \\
(\mathrm{km} / \mathrm{s})\end{array}$ & $\begin{array}{l}P_{\text {orb }} \\
\text { (days) }\end{array}$ & $\begin{array}{c}D \\
(\mathrm{kpc})\end{array}$ & method $^{b}$ & $\underset{(\mathrm{mag})}{E(B-V)}$ \\
\hline V1324 Sco & 357.426 & -2.872 & $<10(\mathrm{~V})(37)$ & $10.14(100)$ & $25(100)$ & $29.5(100)$ & & Fe II (74) & & $2000(74)$ & $0.133(101)$ & $: 4.3 \pm 0.9$ & $\operatorname{rdr}(6)$ & $1.16 \pm 0.12(102)$ \\
\hline V496 Sct & 25.284 & -1.768 & $<11.9$ (V) (37) & $7.07(103)$ & $59(103)$ & $90(103)$ & & Fe II (103) & & 1200 (103) & & $3.2 \pm 0.8$ & $\operatorname{rdr}(6)$ & $0.57 \pm 0.06(6)$ \\
\hline FH Ser & 32.909 & 5.786 & 12.3 (V) (1) & $4.5(1)$ & 49 (1) & $62(1)$ & $\mathrm{D}(1)$ & Fe II (7) & & & & $0.95 \pm 0.05$ & epar (61) & $0.6 \pm 0.1(6)$ \\
\hline LW Ser & 12.959 & 6.047 & 11.1 (V) (1) & $8.3(1)$ & 32 (1) & $52(1)$ & $\mathrm{D}(1)$ & & & & & $2.34 \pm 0.46$ & epar (62) & $0.39 \pm 0.1(6)$ \\
\hline $\mathrm{V} 732 \mathrm{Sgr}^{d}$ & 2.53 & -1.188 & $\sim 9.6$ (V) (1) & $6.4(1)$ & $65(1)$ & 75 (1) & $\mathrm{D}(1)$ & & & & & $3.0 \pm 0.2$ & $\operatorname{rdr}(6)$ & $0.81 \pm 0.16(104)$ \\
\hline V3890 Sgr & 9.204 & -6.443 & 7.4 (V) (1) & $8.1(1)$ & $6(1)$ & $14(1)$ & $\mathrm{S}(1)$ & hybrid (21) & $\mathrm{RN}(2)$ & $2140(105)$ & 519.7 (92) & $7.0 \pm 1.6$ & comp. (2) & $0.9 \pm 0.3(2)$ \\
\hline $\mathrm{V} 4643 \mathrm{Sgr}^{d}$ & 3.345 & -0.337 & $>8.3(\mathrm{~V})(1)$ & $7.7(1)$ & $3(1)$ & $6(1)$ & $\mathrm{S}(1)$ & & cRN (14) & $4700(106)$ & & $3.1 \pm 0.2$ & $\operatorname{rdr}(6)$ & $1.47 \pm 0.2(107)$ \\
\hline V5558 Sgr & 11.611 & 0.207 & $<13.5$ (V) (37) & $8.3(108)$ & $281(108)$ & $473(108)$ & & hybrid (108) & & 1000 (109) & & $2.1 \pm 0.4$ & $\operatorname{rdr}(6)$ & $0.8 \pm 0.1(110)$ \\
\hline XX Tau & 187.104 & -11.653 & $12.6(\mathrm{p})(4)$ & $6(7)$ & $24(7)$ & $43(7)$ & & Fe II (7) & & & & $3.5 \pm 0.7$ & epar (9) & $0.22 \pm 0.03(10)$ \\
\hline V382 Vel & 284.167 & 5.772 & 13.8 (V) (1) & $2.8(1)$ & $6(1)$ & $13(1)$ & $\mathrm{S}(1)$ & $\mathrm{He} / \mathrm{N}(21)$ & & $2400(111)$ & $0.14613(112)$ & $0.8 \pm 0.1$ & epar (113) & $0.12 \pm 0.03(6)$ \\
\hline LV Vul & 63.302 & 0.846 & $10.8(\mathrm{~V})(1)$ & $4.5(1)$ & 20 (1) & $38(1)$ & $\mathrm{s}(1)$ & Fe II (7) & & & & $2.5 \pm 0.5$ & epar (114) & $0.57 \pm 0.05(6)$ \\
\hline NQ Vul & 55.355 & 1.29 & 11 (V) (1) & $6.2(1)$ & $21(1)$ & $50(1)$ & $\mathrm{D}(1)$ & Fe II (7) & & & & $1.6 \pm 0.8$ & epar (29) & $0.92 \pm 0.2(6)$ \\
\hline PW Vul & 61.098 & 5.197 & 10.5 (V) (1) & $6.4(1)$ & 44 (1) & $116(1)$ & $\mathrm{J}(1)$ & Fe II (7) & & & $0.214(115)$ & $1.8 \pm 0.05$ & epar (7) & $0.55 \pm 0.1(107)$ \\
\hline QU Vul & 68.511 & -6.026 & 12.6 (V) (1) & $5.3(1)$ & 20 (1) & $36(1)$ & $\mathrm{P}(1)$ & Fe II (7) & cRN: (14) & & $0.112(4)$ & 3.14 & epar (116) & $0.55 \pm 0.05(117)$ \\
\hline QV Vul & 53.859 & 6.974 & 10.9 (V) (1) & $7.1(1)$ & 37 (1) & 47 (1) & $\mathrm{D}(1)$ & Fe II (7) & & 920 (118) & & $2.7 \pm 0.54$ & epar (7) & $0.4 \pm 0.05$ (6) \\
\hline \multicolumn{15}{|c|}{ 1b. The novae that are not used in MMRD analyses. } \\
\hline $\mathrm{V} 1301 \mathrm{Aql}^{c, e}$ & 40.225 & -3.68 & $10.7(\mathrm{p}, \mathrm{V})(4)$ & $10.3(119)$ & $21(27)$ & $35(119)$ & & & & & & $2.2 \pm 0.3$ & $\operatorname{rdr}(6)$ & $0.61(120)$ \\
\hline $\mathrm{V} 1493 \mathrm{Aql}^{\mathrm{c}}$ & 45.908 & 2.155 & $\sim 10.9$ (V) (1) & $10.1(1)$ & $9(1)$ & $50(1)$ & $\mathrm{C}(1)$ & & cRN: (14) & 3280,3400 (121) & $0.156(4)$ & $1.72 \pm 0.66$ & rdr (6) & $0.57 \pm 0.14(6)$ \\
\hline V868 $\mathrm{Cen}^{f}$ & 309.508 & -1.028 & 11.2 (V) (1) & 8.7 (1) & 31 (1) & $82(1)$ & $\mathrm{J}(1)$ & Fe II (21) & & 700 (122) & & $5.2 \pm 0.4$ & $\operatorname{rdr}(6)$ & $1.72 \pm 0.1(6)$ \\
\hline $\mathrm{V} 888 \mathrm{Cen}^{c}, d$ & 304.311 & 2.649 & $7.2(\mathrm{~V})(1)$ & $8(1)$ & $38(1)$ & $90(1)$ & $\mathrm{O}(1)$ & Fe II (1) & & & & $: 1.28 \pm 0.9$ & rdr (5) & $0.34(123)$ \\
\hline $\mathrm{AR} \mathrm{Cir}^{c, e}$ & 317.039 & -0.372 & $9(\mathrm{~B}, \mathrm{p})(124)$ & $10.5(124)$ & 208 (124) & 330 (124) & & & & & $0.214(125)$ & $3.15 \pm 0.56$ & rdr (6) & $2.0 \pm 0.4(6)$ \\
\hline $\mathrm{BY}^{\mathrm{Cir}^{c}}$ & 315.022 & -3.723 & 10.5 (V) (1) & $7.4(1)$ & $35(1)$ & $124(1)$ & $\mathrm{P}(1)$ & & & $1500(126)$ & $0.282(4)$ & $1.0 \pm 0.2$ & $\operatorname{rdr}(6)$ & $0.13 \pm 0.06(6)$ \\
\hline $\mathrm{V} 394 \mathrm{Cr} A^{c, d}$ & 352.822 & -7.723 & 11.2 (V) (2) & $7.2(2)$ & $2.4(2)$ & $5.2(2)$ & & & $\mathrm{RN}(2)$ & $4600(89)$ & $1.516(92)$ & 2.0 & rdr (6) & $0.2(6)$ \\
\hline $\mathrm{QCyg}^{\mathrm{c}}$ & 89.929 & -7.552 & 12.6 (V) (4) & $3(26)$ & $5(26)$ & $11(26)$ & & & cRN: (14) & & $0.42(11)$ & $1.22 \pm 0.9$ & $\operatorname{rdr}(6)$ & $0.26 \pm 0.06(13)$ \\
\hline HY Lup $c, e$ & 318.536 & 8.629 & 10.9 (V) (4) & $8(127)$ & $15(80)$ & $>23(7)$ & & & & $2700(128)$ & & $1.8 \pm 0.36$ & epar (7) & $0.34 \pm 0.09$ (129) \\
\hline V959 Mon ${ }^{e}$ & 206.341 & 0.076 & 8.1 (V) (37) & & & & & & & & $0.2965(37)$ & $1.4 \pm 0.4$ & epar (130) & $0.38 \pm 0.1(131)$ \\
\hline $\mathrm{V} 972 \mathrm{Oph}^{d, e}$ & 359.376 & 2.428 & 9.5 (B) (4) & $8(26)$ & $50(27)$ & $176(26)$ & & & & & $0.2812(125)$ & $: 0.87 \pm 0.15$ & rdr (6) & $0.5 \pm 0.1(132)$ \\
\hline $\mathrm{V} 2214 \mathrm{Oph}^{c}$ & 355.373 & 5.725 & 12 (V) (1) & $8.5(1)$ & $60(1)$ & 89 (1) & $\mathrm{S}(1)$ & Fe II (21) & & 1025 (133) & $0.118(4)$ & $2.6 \pm 1.0$ & rdr (6) & $0.73 \pm 0.1(6)$ \\
\hline $\mathrm{V} 2576 \mathrm{Oph}^{c, d, e}$ & 356.2 & 5.369 & $<7.8$ (V) (37) & $9.2(134)$ & $20(20)$ & $29(20)$ & & Fe II (74) & & 1470 (134) & & $1.8 \pm 1.0$ & $\operatorname{rdr}(6)$ & $0.62(135)$ \\
\hline $\mathrm{V} 2674 \mathrm{Oph}{ }^{d, e}$ & 357.842 & 3.579 & $<10.7$ (V) (37) & $9.4(136)$ & $18(136)$ & $31(136)$ & & & & & & $1.65 \pm 0.38$ & $\operatorname{rdr}(6)$ & $0.7 \pm 0.1(136)$ \\
\hline $\mathrm{V} 529 \mathrm{Oric}^{\mathrm{c}, e}$ & 188.935 & -1.937 & 13.6 (V) (37) & & & & & & & & & $? 1.29 \pm 0.35$ & rdr (6) & $0.32 \pm 0.02(137)$ \\
\hline $\mathrm{V} 992 \mathrm{Sco} d, f$ & 343.824 & -1.602 & 9.5 (V) (1) & $7.7(1)$ & $100(1)$ & $120(1)$ & $\mathrm{D}(1)$ & Fe II (21) & & 800 (138) & 0.154 (4) & $2.63 \pm 0.35$ & $\operatorname{rdr}(6)$ & $1.3 \pm 0.1(6)$ \\
\hline V977 Sco ${ }^{e}$ & 357.604 & -2.997 & $11.6(\mathrm{j}, \mathrm{p})(4)$ & $9.4(26)$ & & $8(26)$ & & Fe II (21) & & & & $3.3 \pm 0.6$ & rdr (6) & $0.92 \pm 0.1(6)$ \\
\hline ASASSN-17hx $d, e$ & 17.973 & -2.224 & $10.4(\mathrm{G}, \mathrm{V})(37)$ & $10.9(139)$ & & & & Fe II (139) & & $800(140)$ & & $: 3.99 \pm 1.0$ & $\operatorname{rdr}(5)$ & 0.68 (139) \\
\hline $\mathrm{EU} \mathrm{Sct}^{h}$ & 29.727 & -2.98 & 9.6 (p) (4) & $8.4(26)$ & $20(26)$ & $42(26)$ & & & cRN: (14) & & & $5.1 \pm 1.7$ & epar? (141) & $0.84(142)$ \\
\hline $\mathrm{V} 368 \mathrm{Sct}^{c}$ & 24.669 & -2.629 & $12.1(\mathrm{p}, \mathrm{V})(4)$ & $6.9(119)$ & $16(27)$ & $30(119)$ & & & & & & $? 1.94 \pm 0.95$ & rdr (6) & $0.5(143)$ \\
\hline $\mathrm{V} 373 \mathrm{Sct}^{\mathrm{c}}$ & 26.504 & -4.397 & $>12.2(\mathrm{~V})(1)$ & $6.1(1)$ & $47(1)$ & $79(1)$ & $J(1)$ & & & & $0.1536(125)$ & $: 1.1 \pm 1.0$ & $\operatorname{rdr}(6)$ & $0.23 \pm 0.04(144)$ \\
\hline $\mathrm{V} 443 \mathrm{Sct}^{c}$ & 27.218 & -2.421 & $>11.5$ (V) (1) & $8.5(1)$ & $33(1)$ & $60(1)$ & $\mathrm{J}(1)$ & Fe II (21) & & $1400(145)$ & & $: 1.25 \pm 0.7$ & $\operatorname{rdr}(5)$ & $0.4 \pm 0.05(6)$ \\
\hline $\mathrm{V} 476 \mathrm{Sct}^{e}$ & 24.741 & 1.213 & $<6$ (V) (37) & $11.1(146)$ & $15(146)$ & $28(146)$ & & Fe II (21) & & $1200(74)$ & & $11.3 \pm 1.9$ & rdr (6) & $1.9 \pm 0.1(6)$ \\
\hline $\mathrm{CT} \mathrm{Ser}^{e}$ & 24.482 & 47.563 & $8.7(\mathrm{p}, \mathrm{V})(4)$ & $5(26)$ & $100(30)$ & $>100(30)$ & & & cRN: (14) & & 0.195 (4) & $1.4 \pm 0.28$ & epar (7) & $? 0.23$ (142) \\
\hline $\mathrm{MU} \mathrm{Ser} c, e$ & 14.067 & 5.555 & $<12.3$ (V) (37) & $7.7(26)$ & $2.5(8)$ & $5(26)$ & & & & $1230(8)$ & & $: 0.72 \pm 0.53$ & $\operatorname{rdr}(6)$ & $20.4 \pm 0.1(147)$ \\
\hline HM Sge $e, g$ & 53.566 & -3.15 & & $11.3(148)$ & & & & & & & $535.0(37)$ & $1.78 \pm 0.1$ & epar (149) & $0.6 \pm 0.1(150)$ \\
\hline WY Sge $c, e$ & 53.368 & -0.739 & & & & & & & & & & $4.2 \pm 0.4$ & rdr (6) & $1.6 \pm 0.3(6)$ \\
\hline $\mathrm{V} 1016 \mathrm{Sgr}^{c}, e, g$ & 7.033 & -4.807 & $8.5(\mathrm{p})(4)$ & $8.4(26)$ & & $140(26)$ & & & & & & $: 1.5 \pm 1.2$ & $\operatorname{rdr}(5)$ & $0.35 \pm 0.04(151)$ \\
\hline $\mathrm{V} 1172 \mathrm{Sgr}^{c}, e$ & 7.639 & 3.335 & $9(\mathrm{j}, \mathrm{p})(4)$ & $14(24)$ & $6(24)$ & & & & & $2000-3000(152)$ & & $: 0.86 \pm 0.14$ & rdr (6) & $? 0.4(142)$ \\
\hline $\mathrm{V} 3645 \mathrm{Sgr}^{c}, d, e$ & 14.515 & -5.093 & $5.4(\mathrm{p})(4)$ & $8 ?(119)$ & & $300 ?(119)$ & & & & & & $: 1.33 \pm 1.33$ & rdr (5) & $: 0.39(142)$ \\
\hline $\mathrm{V} 3888 \mathrm{Sgr}^{e}$ & 9.085 & 4.658 & 12 (V) (4) & & & & & & & & & 2.5 & epar (7) & $0.82(6)$ \\
\hline $\mathrm{V} 4077 \mathrm{Sgr}^{e}$ & 7.427 & -8.319 & $14(\mathrm{j}, \mathrm{V})(4)$ & $8(26)$ & $20(26)$ & $100(26)$ & & & & & $0.16(30)$ & $1.6 \pm 1.0$ & $\operatorname{rdr}(6)$ & $0.32 \pm 0.03(6)$ \\
\hline $\mathrm{V} 4160 \mathrm{Sgr}^{c}$ & 0.2 & -6.968 & $>12$ (V) (1) & $7(1)$ & $2(1)$ & $3(1)$ & $\mathrm{S}(1)$ & $\mathrm{He} / \mathrm{N}(153)$ & cRN (14) & $4500(154)$ & & $1.85 \pm 1.2$ & rdr (6) & $0.35 \pm 0.04(6)$ \\
\hline $\mathrm{V} 4169 \mathrm{Sgr}^{c}$ & 4.558 & -6.964 & $>9.1$ (V) (1) & $7.9(1)$ & 24 (1) & $36(1)$ & $\mathrm{S}(1)$ & Fe II (21) & & & & $? 1.9 \pm 0.7$ & rdr (6) & $0.35 \pm 0.05(6)$ \\
\hline $\mathrm{V} 4332 \mathrm{Sgr} c, e$ & 13.628 & -9.4 & 9.8 (V) (4) & $8.1(20)$ & $7(20)$ & $10(20)$ & & & & & & $1.14 \pm 1.0$ & rdr (6) & $0.32 \pm 0.07(6)$ \\
\hline
\end{tabular}




\begin{tabular}{|c|c|c|c|c|c|c|c|c|c|c|c|c|c|c|}
\hline Nova & $\begin{array}{c}l \\
(\operatorname{deg})\end{array}$ & $\begin{array}{c}b \\
(\operatorname{deg})\end{array}$ & $\underset{\text { (mag) }}{\text { Amplitude }{ }^{a}}$ & $\begin{array}{r}V_{\max } \\
(\operatorname{mag})\end{array}$ & $\begin{array}{c}t_{2} \\
\text { (days) }\end{array}$ & $\begin{array}{c}t_{3} \\
\text { (days) }\end{array}$ & LC type & Spec type & RN type & $\begin{array}{c}\mathrm{FWHM} \\
(\mathrm{km} / \mathrm{s})\end{array}$ & $\begin{array}{l}P_{\text {orb }} \\
\text { (days) }\end{array}$ & $\begin{array}{c}D \\
(\mathrm{kpc})\end{array}$ & method $^{b}$ & $\begin{array}{c}E(B-V) \\
(\mathrm{mag})\end{array}$ \\
\hline $\mathrm{V} 4444 \mathrm{Sgr}^{\mathrm{c}}$ & 3.818 & -3.389 & $>13.4(\mathrm{~V})(1)$ & $7.6(1)$ & $5(1)$ & $9(1)$ & $\mathrm{S}(1)$ & & cRN? (14) & $800(155)$ & & $2.27 \pm 0.58$ & $\operatorname{rdr}(6)$ & $0.45 \pm 0.1(6)$ \\
\hline $\mathrm{V} 4633 \mathrm{Sgr}^{\mathrm{c}}$ & 5.128 & -6.231 & $11.3(\mathrm{~V})(1)$ & $7.4(1)$ & $17(1)$ & $44(1)$ & P (1) & Fe II (114) & cRN: (14) & $1700(114)$ & $0.12557(156)$ & $: 1.36 \pm 0.46$ & $\operatorname{rdr}(6)$ & $0.26 \pm 0.05(6)$ \\
\hline $\mathrm{v} 5113 \mathrm{Sgr}^{c}, d, e$ & 3.721 & -4.091 & $<9.2$ (V) (37) & & & & & & & $550(157)$ & & $: 0.95 \pm 0.21$ & $\operatorname{rdr}(6)$ & $0.1 \pm 0.02(158)$ \\
\hline $\mathrm{V} 5115 \mathrm{Sgr} c, d$ & 6.046 & -4.567 & $>10.1$ (V) (1) & $7.9(1)$ & $7(1)$ & $13(1)$ & $\mathrm{S}(1)$ & $\mathrm{He} / \mathrm{N}(21)$ & & $1300(159)$ & & $\sim 3.0 \pm 1.0$ & $\operatorname{rdr}(6)$ & $0.53 \pm 0.05(160)$ \\
\hline $\mathrm{V} 5116 \mathrm{Sgr}^{c}$ & 2.136 & -6.833 & $>8.4$ (V) (1) & $7.6(1)$ & 12 (1) & 26 (1) & $\mathrm{S}(1)$ & Fe II (21) & & $970(161)$ & $0.1238(162)$ & $: 1.55 \pm 0.7$ & $\operatorname{rdr}(6)$ & $0.23 \pm 0.06(6)$ \\
\hline $\mathrm{v} 5117 \mathrm{Sgr} d, e$ & 354.624 & -6.377 & $<7.8$ (V) (37) & $8.6(163)$ & $59(164)$ & $83(165)$ & & Fe II (74) & & $1600(74)$ & & $1.45 \pm 0.33$ & $\operatorname{rdr}(6)$ & $0.5 \pm 0.1(166)$ \\
\hline $\mathrm{V} 5588 \mathrm{Sgr}^{c}$ & 7.84 & -1.884 & $<8.7$ (V) (37) & $12.37(167)$ & $38(167)$ & $77(167)$ & & hybrid (167) & & 900 (168) & & $3.13 \pm 0.7$ & $\operatorname{rdr}(5)$ & $1.2 \pm 0.15(169)$ \\
\hline V5589 $\mathrm{Sgr}^{\mathrm{c}}$ & 4.977 & 3.072 & $<7.2$ (V) (37) & $8.8(170)$ & $4.5(74)$ & $7(74)$ & & hybrid (74) & & $5700(74)$ & & $: 3.27 \pm 0.6$ & rdr (5) & $0.8 \pm 0.19$ (171) \\
\hline $\mathrm{RW} \mathrm{UMi}^{e}$ & 109.638 & 33.15 & $12.5(\mathrm{~B}, \mathrm{p})(4)$ & $6(26)$ & & $140(26)$ & & & & & $0.081(30)$ & $5.0 \pm 2.0$ & epar (29) & $0.03 \pm 0.03(10)$ \\
\hline $\mathrm{CK} \mathrm{Vul}^{e}$ & 63.383 & 0.99 & 18.1 (V) (4) & $2.6(26)$ & $40(26)$ & $100(26)$ & & & & & & $4.48 \pm 0.24$ & rdr (6) & $0.75 \pm 0.05(6)$ \\
\hline
\end{tabular}

Notes: A “"." indicates an uncertain value, and " "?" for unreliable value

${ }^{a}$ Photometric band of amplitude are in parenthesis: first and second character for bands of lower and upper limit, respectively. If only one character is given, it is for both lower and upper limit.

$b$ Method for calculation of distance: epar, expansion parallax; tpar, trigonometric parallax; rdr, reddening-distance relation, comp., companion star; mult., multiple models.

${ }^{c}$ Distance of the nova is uncertain owing to i.e. uncertain reddening estimates, differing in large scale, shallow reddening curve

${ }^{d}$ Calculation of distance for the nova depends on only one reddening estimates

${ }^{e}$ The nova have uncertain or missing light curve parameter(s)

(Williams 1994) pulling luminosity of this nova to lower values.

$g$
$h$ Symbiotic nova

The distance measurement depends on various methods, not clearly stated; nebular expansion parallaxes, interstellar line strengths, differential galactic rotation, or several other methods

References: (1) Strope et al. (2010), (2) Schaefer (2010), (3) Kiss et al. (2001), (4) Downes et al. (2001), (5) in this study, (6) Özdönmez et al. (2016), (7) Downes \& Duerbeck (2000), (8) Tappert et al. (2014), (9) Cohen (1985), (10) Green et al. (2015), (11) Peters \& Thorstensen (2006), (12) Gaia Collaboration et al. (2016), (13) Selvelli \& Gilmozzi (2013), (14) Pagnotta \& Schaefer (2014), (15) della Valle \& Duerbeck (1993), (16) Helton et al. (2012), (17) Kiss \& Thomson (2000), (18) Barsukova et (2011), (25) Helton et al. (2008a), (26) Shafter (1997), (27) Warner (1987), (28)

Zhao \& McClintock (1997), (29) Slavin et al. (1995), (30) Diaz \& Bruch (1997), (31) Eyres et al. (1996), (32) Goranskij et al. (2007), (33) Iijima \& Rosino (1996), (34) Lyke \& Campbell (2009), (35) Munari et al. (1996), (36) Sekiguchi et al. (1989), (37) AAVSO database, (38) Helton et al. (2010), (39) Bloch et al. (1946), (40) Munari et al (2016), (41) Lyke et al. (2003), (42) della Valle \& Benetti (1997), (43) Munari et al. (2011a), (44) Iijima (2015), (45) Krautter et al. (1981), (46) Whitney \& Clayton
(1989), (477) Greiner et al. (2003), (48) De Young \& Schmidt (1994). (49) Chochol et al. (1997). (50) Iijima (2001), (51) Kiss et al. (2002), (52) Nakano et al. (2007a), (53) Swierczynskiet al. (2008), (54) Raj et al. (2015a), (55) Schwarz et al. (2009), (56) Naik et al. (2009), (57) Tomov et al. (2008), (58) Munari et al. (201 1b), (59) Harrison et al. (2013), (60) Thorstensen \& Taylor (2000), (61) Gill \& O’Brien (2000), (62) Cohen \& Rosenthal (1983), (63) Katysheva \& Shugarov (2007), (64) Sanford (1940), (65) Gill \& O'Brien (1998), (66) Orio et al. (2005), (67) Woudt \& Warner (2003), (68) Liller et al. (2005a), (69) Hachisu \& Kato (2007), (70) Bode et al. (2007), (71) Snijders (1987), (72) Lynch et al. (2000), (73) Munari et al. (2008a), (74) Walter et al. (2012), (75) Nakano et al. (2007b), (76) Nakano et al. (2009), (77) Munari et al. (2011c), (78) Ayani et al. (2009), (79) Gilmozzi et al. (1994), (80) Woudt \& Warner (2001), (81) Orio et al. (1996), (82) Iijima \& Nakanishi (2008), (83) Woudt et al. (2009), (84) Williams (2013), (85) Catchpole (1969), (86) Schaefer et al. (2013), (87) Gilmozzi \& Selvelli (2007), (88) Mason et al. (2012), (89) Sekiguchi et al. (1988), (90) Maxwell et al. (2012), (91) Williams et al. (1991), (92) Schaefer (2009), (93) Lynch et al. (2006a), (94) Hounsell et al. (2010a), (95) Yamaoka et al. (2007a), (96) Munari et al. (2007a), (97) Naito et al. (2012), (98) Mason et al. (2010), (99) Naito \& Fujii (2008), (100) Munari et al. (2015a), (101) Wagner et al. (2012), (102) Finzell et al. (2015), (103) Raj et al. (2012), (104) Weight et al. (1993), (105) Wagner et al. (1990), (106) Ayani et al. (2001), (107) Andreae et al. (1991), (108) Das et al. (2015), (109) lijima et al. (2007), (1108) Rudy et al. (2007), (111) Della Valle et al. (2002), (112) (1198) Balman et al. (2006), (12) Tomov et al. (2015), (114) della Valle et al. (1998), (11) Hacke (1987), (116) Krauter et al. (2002), (117) Hachisu \& Kato (2014), (118) Gallagher et al. (1987), (119) Duerbeck (1987), (120) Viba et al. (1977), (121)

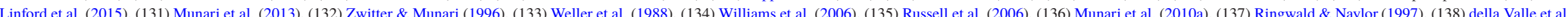
(1992b), (139) Munari et 2l (2017b), (140) Williams \& Darnley (2017). (141) Duerbeck (1981), (142) Weightet al (1994), (143) Ciatti \& Rosino (1974), (144) Miroshnichenk (1988), (145) Barbon et al (1989), (146) Munari et al (2006a), (147) Kosai et al. (1983). (148) Belyakina et al. (1988). (149) Hack \& Paresce (1993), (150) Nuschaumer \& Vogel (1990). (151) Birriel et al (2000), (152) Strömgren \& Shapley (1951), (153) Williams et al. (1994), (154) Kingsburgh et al (1991), (155) Garnavich et al. (1999), (156) Lipkin \& Leibowitz (2008), (157) Brown et al. (2003), (158) Ruch et al. (2003), (159) Ayani \& Kawahata (2005), (160) Rudy et al. (2005), (161) Liller (2005), (162) Dobrotka et al. (2008), (163) Liller et al. (2006), (164) Senziani et al. (2008), (165) Williams et al. (2008), (166) Lynch et al. (2006b), (167) Munari et al. (2015b), (168) Arai et al. (2011), (169) Rudy et al. (2011), (170) Korotkiy et al. (2012), (171) Weston et al. (2016) 
Table 6. The Galactic novae whose distances could not be estimated. Lower limits of distances for the novae, calculated from reddening-distance relations, are given as well. The column definitions are the same as

\begin{tabular}{|c|c|c|c|c|c|c|c|c|c|c|c|c|c|}
\hline Nova & $\begin{array}{c}l \\
\text { (deg) }\end{array}$ & $\begin{array}{c}b \\
(\operatorname{deg})\end{array}$ & $\underset{\text { (mag) }}{\text { Amplitude }^{a}}$ & $\begin{array}{r}V_{\max } \\
(\operatorname{mag})\end{array}$ & $\begin{array}{c}t_{2} \\
\text { (days) }\end{array}$ & $\begin{array}{c}t_{3} \\
\text { (days) }\end{array}$ & LC type & Spec type & RN type & $\begin{array}{c}\text { FWHM } \\
(\mathrm{km} / \mathrm{s})\end{array}$ & $\begin{array}{l}P_{\text {orb }} \\
\text { (days) }\end{array}$ & $\begin{array}{c}D \\
(\mathrm{kpc})\end{array}$ & $\underset{(\mathrm{mag})}{E(B-V)}$ \\
\hline OS And & 106.051 & -12.117 & 11 (V) (1) & $6.5(1)$ & $11(1)$ & $23(1)$ & $\mathrm{D}(1)$ & & & 1740 (2) & & & $0.15 \pm 0.05$ \\
\hline DO Aql & 31.705 & -11.806 & $9.5(\mathrm{~V})(1)$ & $8.5(1)$ & $295(1)$ & $900(1)$ & $\mathrm{F}(1)$ & & & & $0.16776(4)$ & & \\
\hline V1370 Aql & 38.813 & -5.947 & $10.3(\mathrm{~V})(1)$ & $7.7(1)$ & $15(1)$ & $28(1)$ & $\mathrm{D}(1)$ & & & $730(5)$ & & $\geqslant 1.6(6)$ & $0.35 \pm 0.05$ \\
\hline V1419 Aql & 36.811 & -4.1 & $\sim 13.4$ (V) (1) & $7.6(1)$ & $25(1)$ & $32(1)$ & $\mathrm{D}(1)$ & & & & & $\geqslant 4.7(6)$ & $0.5 \pm 0.05$ (3) \\
\hline V1425 Aql & 33.011 & -3.893 & $\sim 8(\mathrm{~V})(1)$ & $8.0(1)$ & $27(1)$ & $79(1)$ & $S(1)$ & Fe II (1) & & $1340(8)$ & $0.2558(4)$ & $\geqslant 2.2(6)$ & $1.0 \pm 0.3(9)$ \\
\hline V356 Aql & 37.418 & -4.944 & 11.3 (V) (1) & $7.0(1)$ & $127(1)$ & $140(1)$ & $J(1)$ & Fe II (1) & cRN? (10) & $1140(5)$ & & & \\
\hline V528 Aql & 36.687 & -5.91 & $11.6(\mathrm{~V})(1)$ & $6.9(1)$ & $16(1)$ & $38(1)$ & $S(1)$ & & & 915 (11) & & & \\
\hline V834 Car & 290.18 & -4.282 & $<8.8$ (V) (4) & $10.2(12)$ & $20(13)$ & $38(13)$ & & Fe II (13) & & & & & \\
\hline N Cen 2012b & 314.833 & 1.931 & & $9.5(13)$ & $12.3(13)$ & $19.8(13)$ & & Fe II (13) & & & & & \\
\hline V1039 Cen & 309.837 & -2.259 & $\sim 11.7$ (V) (1) & $9.3(1)$ & $25(1)$ & $174(1)$ & $J(1)$ & & & & 0.247 (4) & & \\
\hline V1368 Cen & 309.446 & 3.979 & $<8.4$ (V) (4) & $9.4(13)$ & $16(13)$ & 34 (13) & & Fe II (13) & & & & & \\
\hline IV Cep & 99.614 & -1.638 & 9.7 (B) (14) & $7.0(15)$ & $11(15)$ & $37(15)$ & & & & & & $\geqslant 5.7(6)$ & $0.65 \pm 0.05(7)$ \\
\hline V809 Cep & 110.647 & 0.399 & $<8.7$ (V) (4) & $11.181(16)$ & $16(16)$ & $36(16)$ & & Fe II (16) & & $500(16)$ & & $\geqslant 7.0(6)$ & $1.7(16)$ \\
\hline V962 Cep & 97.314 & 9.819 & $<10$ (V) (4) & $11.5(17)$ & $22(17)$ & $42(17)$ & & Fe II (17) & & & & & $0.94(17)$ \\
\hline DD Cir & 310.997 & -7.727 & $12.6(\mathrm{~V})(1)$ & $7.6(1)$ & $5(1)$ & $16(1)$ & $\mathrm{P}(1)$ & & cRN: (10) & & 0.0975 (4) & & \\
\hline V693 CrA & 357.83 & -14.391 & $>14(\mathrm{~V})(1)$ & $7.0(1)$ & $10(1)$ & $18(1)$ & $\mathrm{S}(1)$ & & cRN: (10) & 4500 (18) & & & $0.05 \pm 0.05(19)$ \\
\hline V1330 Cyg & 78.376 & -5.491 & 7.6 (V) (1) & $9.9(1)$ & $161(1)$ & $217(1)$ & $\mathrm{S}(1)$ & & & & & & \\
\hline V1668 Cyg & 90.837 & -6.76 & 13.5 (V) (1) & $6.2(1)$ & $11(1)$ & $26(1)$ & $\mathrm{S}(1)$ & Fe II (20) & & $2400(21)$ & $0.1384(4)$ & & $0.38 \pm 0.08(22)$ \\
\hline V2362 Cyg & 87.372 & -2.357 & $>12.9$ (V) (1) & $8.1(1)$ & $9(1)$ & $246(1)$ & $\mathrm{C}(1)$ & Fe II (23) & & $1850(24)$ & $0.06577(25)$ & $\geqslant 6.0(6)$ & $0.56(23)$ \\
\hline V339 Del & 62.199 & -9.423 & 13.3 (V) (4) & $4.4(26)$ & $10(26)$ & $18(26)$ & & Fe II (27) & & $1421(27)$ & & & $0.18 \pm 0.04(26)$ \\
\hline KT Eri & 207.986 & -32.02 & & $5.42(28)$ & $6.6(28)$ & $13.6(29)$ & $P(10)$ & $\mathrm{He} / \mathrm{N}(30)$ & cRN (10) & $3200-3400(31)$ & & & $0.08(32)$ \\
\hline DM Gem & 185.127 & 11.728 & 11.9 (V) (14) & $4.8(15)$ & $6(15)$ & $22(15)$ & & & & & $0.123(4)$ & & \\
\hline DN Gem & 184.018 & 14.714 & $12(\mathrm{~V})(1)$ & $3.6(1)$ & $16(1)$ & $35(1)$ & $\mathrm{P}(1)$ & Fe II (1) & cRN: (10) & & $0.12784(4)$ & & $0.17 \pm 0.02(33)$ \\
\hline V827 Her & 45.808 & 8.594 & 10.6 (V) (1) & $7.5(1)$ & $21(1)$ & $53(1)$ & $\mathrm{s}(1)$ & & & & & & $0.1(34)$ \\
\hline V838 Her & 43.316 & 6.619 & $13.8(\mathrm{~V})(1)$ & $5.3(1)$ & $1(1)$ & $4(1)$ & $\mathrm{P}(1)$ & $\mathrm{He} / \mathrm{N}(35)$ & cRN (10) & $5000(36)$ & $0.29764(37)$ & $\geqslant 2.5(6)$ & $0.5 \pm 0.1(38)$ \\
\hline HR Lyr & 59.584 & 12.47 & $9.3(\mathrm{p})(14)$ & $6.5(15)$ & $45(15)$ & $97(39)$ & & & cRN: (10) & & & & $0.18 \pm 0.06(33)$ \\
\hline LZ Mus & 297.253 & -3.312 & $>9.5$ (V) (1) & $8.5(1)$ & $4(1)$ & $12(1)$ & $\mathrm{P}(1)$ & & cRN: (10) & & & & \\
\hline V2264 Oph & 358.777 & 5.863 & $>11$ (V) (1) & $10.0(1)$ & 22 (1) & $30(1)$ & $\mathrm{S}(1)$ & Fe II (35) & & $2300(40)$ & & & \\
\hline V2295 Oph & 2.378 & 6.973 & $>11.7$ (V) (1) & $9.3(1)$ & $9(1)$ & $16(1)$ & $\mathrm{F}(1)$ & Fe II (35) & & & & $\geqslant 1.4(6)$ & $0.75 \pm 0.2(41)$ \\
\hline V2313 Oph & 6.999 & 6.976 & $>12.5(\mathrm{~V})(1)$ & $7.5(1)$ & $8(1)$ & $17(1)$ & $\mathrm{S}(1)$ & & & $2500(42)$ & & & \\
\hline V2540 Oph & 9.75 & 8.152 & $>12.9$ (V) (1) & $8.1(1)$ & 66 (1) & 115 (1) & $\mathrm{J}(1)$ & & & & $0.28478(43)$ & & \\
\hline V2673 Oph & 5.493 & 4.958 & $<11.7(\mathrm{c}, \mathrm{V})(4)$ & $8.5(44)$ & $10(44)$ & $23.5(44)$ & & & & & & $\geqslant 1.2(45)$ & $0.71(44)$ \\
\hline V3661 Oph & 358.329 & 1.471 & $<11.4(\mathrm{Ic}, \mathrm{R})(4)$ & $10.79(46)$ & $3.9(46)$ & $5.7(46)$ & & Fe II (47) & & & & $\geqslant 4.7(45)$ & $2.37(46)$ \\
\hline V849 Oph & 39.233 & 13.48 & $11.2(\mathrm{~V})(1)$ & $7.6(1)$ & $140(1)$ & $270(1)$ & $\mathrm{F}(1)$ & Fe II (1) & & & $0.17276(4)$ & & \\
\hline V574 Pup & 242.57 & -1.993 & 10.2 (V) (1) & $7.0(1)$ & $12(1)$ & $27(1)$ & $\mathrm{S}(1)$ & Fe II (48) & cRN: (10) & $2830(49)$ & 0.0472: (4) & $\geqslant 6.0(6)$ & $0.5(50)$ \\
\hline T Sco & 352.675 & 19.462 & $<15.2(\mathrm{~B}, \mathrm{~V})(14)$ & $7.0(15)$ & $9(15)$ & $21(51)$ & & & & & & & $0.35(52)$ \\
\hline V1141 Sco & 0.003 & -2.171 & 9.1 (V) (14) & $10.5(53)$ & $17(53)$ & $46(53)$ & & & cRN: (10) & $3200(54)$ & & & \\
\hline V1186 Sco & 354.403 & 4.81 & $>8.3$ (V) (1) & $9.7(1)$ & $12(1)$ & $62(1)$ & $J(1)$ & Fe II (35) & & $500(55)$ & & & \\
\hline V1188 Sco & 355.303 & -2.557 & $>10.1$ (V) (1) & $8.9(1)$ & $11(1)$ & $23(1)$ & $\mathrm{S}(1)$ & Fe II (13) & cRN: (10) & $1730(56)$ & & & \\
\hline V1534 Sco & 354.334 & 3.992 & $7(\mathrm{R})(4)$ & $11.55(46)$ & $5.6(46)$ & $9.2(46)$ & & $\mathrm{He} / \mathrm{N}(57)$ & & $7000(46)$ & & $\geqslant 2.0(45)$ & $1.11(46)$ \\
\hline V1535 Sco & 349.898 & 3.938 & $7.3(\mathrm{~V})(4)$ & $9.3(46)$ & $13.9(46)$ & $21.1(46)$ & & $\mathrm{He} / \mathrm{N}(17)$ & & & & $\geqslant 2.0(45)$ & $1.03(46)$ \\
\hline EU Sct & 29.727 & -2.98 & $9.6(\mathrm{p})(14)$ & $8.4(15)$ & $20(15)$ & $42(15)$ & & & cRN: (10) & & & $>8.96(45)$ & $0.84(58)$ \\
\hline V444 Sct & 25.012 & -2.854 & $9.5(\mathrm{r}, \mathrm{V})(14)$ & $11.4(53)$ & $9(53)$ & $23(53)$ & & & & & & $\geqslant 5.0(6)$ & $1.05(59)$ \\
\hline V475 Sct & 24.201 & -3.947 & $<7.6$ (V) (4) & $8.6(53)$ & $50(53)$ & $55(53)$ & & Fe II (60) & & 1350 (13) & & $\geqslant 5.2(6)$ & $0.69 \pm 0.05(60)$ \\
\hline HS Sge & 54.471 & -1.931 & 13.5 (V) (1) & $7.2(1)$ & $15(1)$ & $21(1)$ & $\mathrm{P}(1)$ & & & $1600(11)$ & & & \\
\hline V4021 Sgr & 10.556 & -7.696 & 9.1 (V) (1) & $8.9(1)$ & $56(1)$ & $215(1)$ & $\mathrm{P}(1)$ & & & & & & $0.45 \pm 0.03(61)$ \\
\hline V4157 Sgr & 5.322 & -3.066 & $<14(\mathrm{j}, \mathrm{p})(14)$ & $9.6(53)$ & $18(53)$ & $41(53)$ & & & & & & $\geqslant 4.8(6)$ & $1.0(59)$ \\
\hline V4171 Sgr & 9.38 & -4.54 & $12.5(\mathrm{j}, \mathrm{p})(14)$ & $6.9(53)$ & $10(53)$ & $20(53)$ & & & & $1200(62)$ & & $\geqslant 2.9(6)$ & $0.61(59)$ \\
\hline V4327 Sgr & 2.477 & -5.425 & $<4(\mathrm{~V})$ & $8.4(53)$ & $19(53)$ & $30(53)$ & & & cRN: (10) & 2400 (63) & & & \\
\hline V4361 Sgr & 13.701 & -2.277 & $6.3(\mathrm{~V}, \mathrm{p})(14)$ & $10.3(53)$ & $52(53)$ & $86(53)$ & & & cRN: (10) & & & & \\
\hline V4642 Sgr & 8.991 & 2.83 & $<8.6$ (V) (14) & $11.8(53)$ & $83(53)$ & $125(53)$ & & & & & & $\geqslant 4.5(6)$ & $1.51 \pm 0.16(64)$ \\
\hline V4739 Sgr & 3.211 & -7.966 & $>10.8$ (V) (1) & $7.2(1)$ & $2(1)$ & $3(1)$ & $\mathrm{S}(1)$ & $\mathrm{He} / \mathrm{N}(65)$ & cRN (10) & $5510(65)$ & & $\geqslant 1.8(6)$ & $0.46 \pm 0.04(66)$ \\
\hline V4740 Sgr & 1.456 & -5.708 & $>11.3$ (V) (1) & $6.7(1)$ & $18(1)$ & $33(1)$ & $\mathrm{s}(1)$ & & & & & & \\
\hline V4741 Sgr & 359.899 & -3.675 & $<8.8(\mathrm{~V})(14)$ & $9.9(53)$ & $10(53)$ & $28(53)$ & & & & $2800(67)$ & & & \\
\hline V4742 Sgr & 4.986 & -1.39 & $>10.1(\mathrm{~V})(1)$ & $7.9(1)$ & $9(1)$ & $23(1)$ & $S(1)$ & Fe II (68) & cRN: (10) & $2500(68)$ & $2(4)$ & & \\
\hline
\end{tabular}




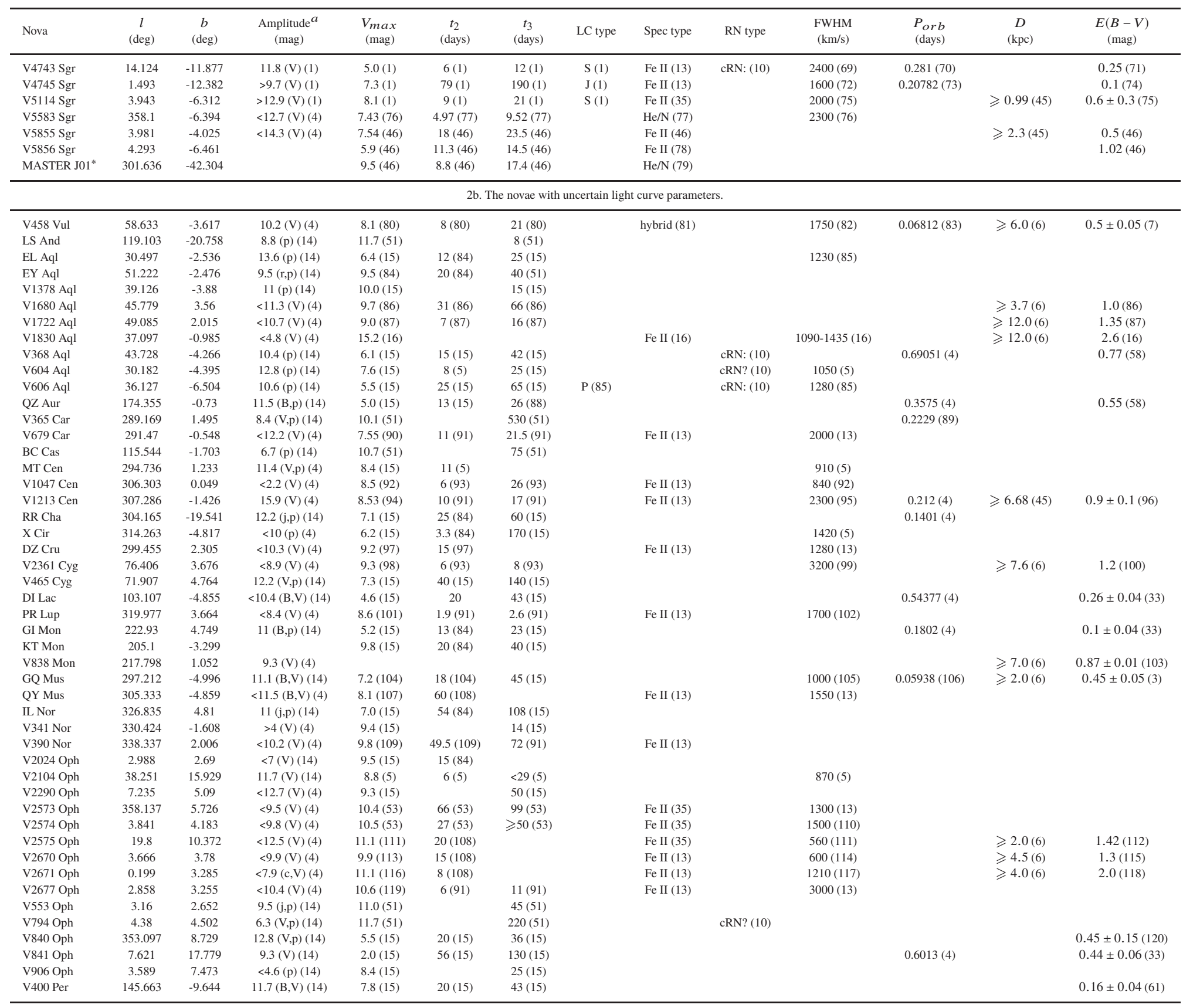




\begin{tabular}{|c|c|c|c|c|c|c|c|c|c|c|c|c|c|}
\hline Nova & $\begin{array}{c}l \\
(\mathrm{deg})\end{array}$ & $\begin{array}{c}b \\
(\operatorname{deg})\end{array}$ & $\underset{(\text { mag) }}{\text { Amplitude }^{a}}$ & $\begin{array}{r}V_{\max } \\
(\operatorname{mag})\end{array}$ & $\begin{array}{c}t_{2} \\
\text { (days) }\end{array}$ & $\begin{array}{c}t_{3} \\
\text { (days) }\end{array}$ & LC type & Spec type & RN type & $\begin{array}{c}\text { FWHM } \\
(\mathrm{km} / \mathrm{s})\end{array}$ & $\begin{array}{l}P_{\text {orb }} \\
\text { (days) }\end{array}$ & $\begin{array}{c}D \\
(\mathrm{kpc})\end{array}$ & $\begin{array}{c}E(B-V) \\
(\mathrm{mag})\end{array}$ \\
\hline DY Pup & 245.823 & 4.362 & $12.6(\mathrm{~V}, \mathrm{p})(14)$ & $7.0(15)$ & $118(84)$ & $160(15)$ & & & & & $0.139(4)$ & & \\
\hline HS Pup & 247.756 & -2.112 & 10.8 (B) (14) & $8.0(15)$ & $33(84)$ & $65(15)$ & & & & & $0.2671(89)$ & & \\
\hline HZ Pup & 246.179 & 1.385 & 9.3 (B) (14) & $7.7(15)$ & $35(84)$ & $70(15)$ & & & & & $0.213(4)$ & & \\
\hline V597 Pup & 252.53 & 0.545 & $13.8(\mathrm{~V})(4)$ & $7.0(121)$ & $2.5(122)$ & & & $\mathrm{He} / \mathrm{N}(122)$ & & $1800(123)$ & 0.11119 & $\geqslant 8.0(6)$ & $0.3(125)$ \\
\hline V598 Pup & 249.108 & -13.814 & 13 (V) (4) & $3.46(29)$ & $9(126)$ & & & & & & & & $0.09 \pm 0.08(126)$ \\
\hline KP Sco & 354.058 & -3.297 & $11.6(\mathrm{j}, \mathrm{p})(14)$ & $9.4(15)$ & $21(84)$ & $38(15)$ & & & & & & & \\
\hline V1142 Sco & 359.289 & -2.893 & $<10.3(\mathrm{~V})(14)$ & $8.5(53)$ & $11(53)$ & $\geqslant 25(53)$ & & & & $3800(127)$ & & & \\
\hline V1281 Sco & 348.856 & 4.798 & $<11$ (V) (4) & $8.8(128)$ & $15(108)$ & & & & & $1800(129)$ & & & $0.7(130)$ \\
\hline V1311 Sco & 346.519 & 3.392 & $<9.5(\mathrm{~V})(4)$ & $8.6(131)$ & $3(91)$ & $6(91)$ & & $\mathrm{He} / \mathrm{N}$ ? (13) & & $3600(13)$ & & & \\
\hline V1312 Sco & 346.067 & 3.038 & $<9.5(\mathrm{~V})(4)$ & $10.5(132)$ & $11(91)$ & $21(91)$ & & Fe II (13) & & $1800(13)$ & & & \\
\hline V696 Sco & 354.887 & -4.909 & $12(\mathrm{j}, \mathrm{p})(14)$ & $7.5(15)$ & & $9(15)$ & & & cRN: (10) & & & & \\
\hline V697 Sco & 353.333 & -5.387 & $9.8(\mathrm{c}, \mathrm{p})(14)$ & $8.0(15)$ & $8(5)$ & $15(15)$ & & & cRN: (10) & $1550(5)$ & $0.1871(4)$ & & \\
\hline V707 Sco & 353.713 & -4.486 & $10.1(\mathrm{j}, \mathrm{p})(14)$ & $9.6(15)$ & & $49(15)$ & & & & & & & \\
\hline V719 Sco & 355.674 & -2.664 & $10.7(\mathrm{j}, \mathrm{p})(14)$ & $9.8(15)$ & & $24(15)$ & & & & $1800(133)$ & & & \\
\hline V720 Sco & 355.15 & -4.468 & 13.5 (p) (14) & $7.5(15)$ & $9(84)$ & & & & & & & & \\
\hline V721 Sco & 354.758 & -2.439 & $<7.9(\mathrm{j}, \mathrm{p})(14)$ & $8.0(15)$ & $120(84)$ & & & & & & & & \\
\hline V722 Sco & 355.167 & -3.662 & $<3.6(\mathrm{~V})(14)$ & $9.4(15)$ & & $18(15)$ & & & & & & & \\
\hline V723 Sco & 354.946 & -4.143 & $9.2(\mathrm{j}, \mathrm{p})(14)$ & $9.8(15)$ & $11(84)$ & $17(15)$ & & & cRN: (10) & & & & \\
\hline V902 Sco & 349.294 & -2.069 & $9(\mathrm{j}, \mathrm{p})(14)$ & $11.0(51)$ & & $200(51)$ & & & & & & & \\
\hline V960 Sco & 358.72 & -3.506 & $9.5(\mathrm{j}, \mathrm{p})(14)$ & $10.5(51)$ & & & & & & & & $\geqslant 4.2(6)$ & $1.3(134)$ \\
\hline FS Sct & 28.895 & -3.978 & $9.2(\mathrm{~B}, \mathrm{p})(14)$ & $10.1(51)$ & & $86(51)$ & & & cRN: (10) & & & & \\
\hline V463 Sct & 17.838 & -2.917 & $<11.1(\mathrm{~V}, \mathrm{p})(14)$ & $11.9(53)$ & $11(53)$ & $>23(53)$ & & & & 990 (135) & & $\geqslant 4.0(6)$ & $0.8 \pm 0.1(135)$ \\
\hline V477 Sct & 20.568 & -2.789 & $<8.6(\mathrm{~V})(4)$ & $9.8(136)$ & $3(136)$ & $6(136)$ & & Fe II (13) & cRN (10) & $2900(137)$ & & $\geqslant 4.0(6)$ & $1.2(138)$ \\
\hline RT Ser & 13.895 & 9.971 & $6.9(\mathrm{p}, \mathrm{V})(4)$ & $10.6(51)$ & & & & & & & & $\geqslant 1.2(6)$ & $0.64 \pm 0.1(139)$ \\
\hline V378 Ser & 14.167 & 7.428 & $<7.7(\mathrm{p}, \mathrm{V})(14)$ & $11.6(93)$ & $44(93)$ & $90(93)$ & & Fe II (35) & & $1100(140)$ & & $\geqslant 4.0(6)$ & $0.74(141)$ \\
\hline $\mathrm{X}$ Ser & 10.841 & 31.872 & $9.4(\mathrm{p})(14)$ & $8.9(84)$ & $400(84)$ & & & & cRN: (10) & & 1.478 (4) & & $0.25(142)$ \\
\hline AT Sgr & 4.127 & -2.172 & $8(\mathrm{j}, \mathrm{p})(14)$ & $8.7(84)$ & $18(84)$ & $35(51)$ & & & & & & & \\
\hline BS Sgr & 5.997 & -7.062 & $8.7(\mathrm{~V}, \mathrm{p})(11)$ & $9.2(51)$ & & $700(51)$ & $F(11)$ & & & $640(11)$ & & & \\
\hline FL Sgr & 356.707 & -5.594 & $11.7(\mathrm{j}, \mathrm{p})(14)$ & $8.0(15)$ & $16(84)$ & $32(15)$ & & & & & & & \\
\hline FM Sgr & 8.118 & -3.547 & $11.9(\mathrm{j}, \mathrm{p})(14)$ & $8.0(15)$ & $15(84)$ & $30(15)$ & & & & & & & \\
\hline HS Sgr & 11.11 & -4.783 & $5(\mathrm{p})(14)$ & $7.0(51)$ & & $20(51)$ & & & & & & & \\
\hline KY Sgr & 3.943 & -1.719 & $9.4(\mathrm{j}, \mathrm{p})(14)$ & $8.0(15)$ & & $60(15)$ & & & & & & & \\
\hline V1012 Sgr & 359.805 & -5.253 & $12(\mathrm{j}, \mathrm{p})(14)$ & $8.0(84)$ & & $32(51)$ & & & & & & & \\
\hline V1014 Sgr & 3.639 & -3.274 & $9.1(\mathrm{j}, \mathrm{p})(14)$ & $10.9(51)$ & & $>50(51)$ & & & & & & & \\
\hline V1015 Sgr & 359.442 & -6.126 & $13.9(\mathrm{j}, \mathrm{p})(14)$ & $6.5(15)$ & $17(84)$ & $34(15)$ & & & & & & & \\
\hline V1017 Sgr & 4.491 & -9.109 & 6.5 (V) (14) & $7.2(15)$ & & $130(15)$ & & & & & $5.714(4)$ & & $0.39 \pm 0.03(143)$ \\
\hline V1059 Sgr & 22.306 & -8.231 & $13.2(\mathrm{~B}, \mathrm{p})(14)$ & $2.0(15)$ & $10(84)$ & & & & & & $0.2861(4)$ & & \\
\hline V1149 Sgr & 4.124 & -5.962 & $13.6(\mathrm{j}, \mathrm{p})(14)$ & $7.4(15)$ & & $210(15)$ & & & & $960(85)$ & & & \\
\hline V1150 Sgr & 7.893 & -4.086 & $<8.7(\mathrm{j}, \mathrm{p})(14)$ & $12.0(51)$ & & $<600(51)$ & & & & & & & \\
\hline V1151 Sgr & 12.045 & -3.597 & $8.9(\mathrm{j}, \mathrm{p})(14)$ & $11.1(51)$ & & $135(51)$ & & & & & & & \\
\hline V1175 Sgr & 1.175 & -6.47 & $<5(\mathrm{p})(14)$ & $7.0(15)$ & $15(84)$ & & & & & & & & \\
\hline V1274 Sgr & 9.885 & 5.068 & $<2.5(\mathrm{p})(14)$ & $10.4(5)$ & $>20(5)$ & $>38(5)$ & & & & $1000(5)$ & & & \\
\hline V1275 Sgr & 355.069 & -6.182 & $11(\mathrm{j}, \mathrm{p})(14)$ & $7.0(15)$ & & $10(15)$ & & & cRN: (10) & & & & \\
\hline V1310 Sgr & 4.16 & -9.977 & $<3.8(\mathrm{p})(14)$ & $11.7(51)$ & & $390(51)$ & & & & & & & \\
\hline V1583 Sgr & 8.138 & -3.051 & $12.1(\mathrm{j}, \mathrm{p})(14)$ & $8.9(15)$ & $20(84)$ & $37(15)$ & & & & $780(85)$ & & & \\
\hline V2572 Sgr & 1.514 & -10.422 & $11.5(\mathrm{j}, \mathrm{p})(14)$ & $6.5(15)$ & $20(84)$ & $44(15)$ & & & & & & & \\
\hline V363 Sgr & 7.63 & -17.072 & $11.2(\mathrm{j}, \mathrm{p})(14)$ & $8.7(15)$ & $22(5)$ & $64(15)$ & & & & $685(5)$ & & & \\
\hline V3889 Sgr & 1.916 & -2.112 & $12.6(\mathrm{j}, \mathrm{V})(14)$ & $8.4(15)$ & $5(84)$ & $14(15)$ & & & & & & & \\
\hline V3964 Sgr & 10.389 & 5.145 & $<7.6(\mathrm{p})(14)$ & $6.0(15)$ & & $32(15)$ & & & & $1320(85)$ & & & \\
\hline V 4074 Sgr & 1.593 & -6.689 & 3.6 (p) (4) & $8.6(51)$ & & $120(51)$ & & & & & & & \\
\hline V4121 Sgr & 2.545 & -4.166 & $11.5(\mathrm{j}, \mathrm{V})(14)$ & $9.5(15)$ & & $60(15)$ & & & & & & & \\
\hline V4135 Sgr & 358.667 & -4.313 & $11.5(\mathrm{j}, \mathrm{V})(14)$ & $10.0(15)$ & & $60(15)$ & & & & $1000(144)$ & & & \\
\hline V441 Sgr & 7.0 & -5.378 & $7.8(\mathrm{p})(4)$ & $8.0(15)$ & & $53(15)$ & & & & & & & \\
\hline V5579 Sgr & 3.734 & -3.023 & $<16.5$ (V) (4) & $6.65(145)$ & $8(145)$ & $13(91)$ & & Fe II (13) & & $1500(108)$ & & $\geqslant 4.1(6)$ & $0.72 \pm 0.06(145)$ \\
\hline V5584 Sgr & 16.168 & -3.1 & $<11.8(\mathrm{~V})(4)$ & $9.2(146)$ & $25(146)$ & $46(146)$ & $\mathrm{D}(146)$ & Fe II (13) & & $600(147)$ & & & \\
\hline V5591 Sgr & 7.223 & 2.538 & $<6.8(\mathrm{~V})(4)$ & $9.7(119)$ & $2(91)$ & $7.5(91)$ & & $\mathrm{He} / \mathrm{N}(13)$ & & $4200(13)$ & & & \\
\hline
\end{tabular}


Table 6 - continued

\begin{tabular}{|c|c|c|c|c|c|c|c|c|c|c|c|c|c|}
\hline Nova & $\begin{array}{c} \\
(\mathrm{deg})\end{array}$ & $\begin{array}{c}b \\
\text { (deg) }\end{array}$ & $\begin{array}{c}\text { Amplitude }^{a} \\
\text { (mag) }\end{array}$ & $\begin{array}{c}V_{\max } \\
(\operatorname{mag})\end{array}$ & $\begin{array}{c}t_{2} \\
\text { (days) }\end{array}$ & $\begin{array}{c}t_{3} \\
\text { (days) }\end{array}$ & LC type & Spec type & RN type & $\begin{array}{c}\text { FWHM } \\
(\mathrm{km} / \mathrm{s})\end{array}$ & $\begin{array}{l}P_{\text {orb }} \\
\text { (days) }\end{array}$ & $\begin{array}{c}D \\
(\mathrm{kpc})\end{array}$ & $\begin{array}{c}E(B-V) \\
(\mathrm{mag})\end{array}$ \\
\hline V5668 Sgr & 5.38 & $\begin{array}{l}-9.867 \\
\end{array}$ & 11.9 (V) (4) & $4.32(148)$ & $100(148)$ & & & Fe II (148) & & & & & \\
\hline V $630 \mathrm{Sgr}$ & 357.768 & -6.967 & & $1.6(15)$ & $4(15)$ & $11(15)$ & & & & & & & \\
\hline $\mathrm{V} 726 \mathrm{Sgr}$ & 5.479 & -5.519 & & $10.5(51)$ & $45(84)$ & $95(51)$ & & & & & 0.41141 (149) & & \\
\hline V737 Sgr & 2.531 & -3.982 & 8.7 (j.p) (14) & $10.7(51)$ & & $>70(51)$ & & & & & & & \\
\hline $\mathrm{V} 787 \mathrm{Sgr}$ & 0.235 & -3.495 & $11.2(\mathrm{j}, \mathrm{p})(14)$ & $9.8(15)$ & $23(84)$ & $74(15)$ & & & & & & & \\
\hline V909 Sgr & 358.767 & -10.403 & $13.2(\mathrm{j}, \mathrm{p})(14)$ & $6.8(15)$ & $3.8(84)$ & $7(15)$ & & & & & $0.14286(89)$ & & \\
\hline V927 Sgr & 358.532 & -6.3 & $12(\mathrm{j}, \mathrm{p})(14)$ & $7.3(15)$ & & $15(15)$ & & & & & & & \\
\hline V928 Sgr & 4.341 & -5.969 & $11.6(\mathrm{j}, \mathrm{p})(14)$ & $8.5(15)$ & $88(5)$ & $150(15)$ & & & & $690(5)$ & & & \\
\hline $\begin{array}{l}\text { V982 Sgr } \\
\text { V } 990 \text { Sgr }\end{array}$ & $\begin{array}{l}4.341 \\
1.84\end{array}$ & $\begin{array}{r}-5.909 \\
-1.889\end{array}$ & $\begin{array}{l}11.0(\mathrm{~d}, \mathrm{P})(14) \\
9.2 \mathrm{~g}, \mathrm{p})(14)\end{array}$ & 11.1(51) & & $24(51)$ & & & & & & & \\
\hline V999 Sgr & 2.811 & $\begin{array}{l}-1.003 \\
-2.042\end{array}$ & $9.4(\mathrm{~B}, \mathrm{p})(11)$ & $7.8(51)$ & & $160(51)$ & & & & $595(11)$ & $0.15184(4)$ & & \\
\hline $\mathrm{RR}$ Tel & 342.163 & -32.242 & 10 (p) (4) & $6.8(51)$ & $670(84)$ & $>2000$ (51) & & & & & & & $0.1 \pm 0.06(150)$ \\
\hline NR TrA & 325.932 & -7.218 & $<10.7(\mathrm{R}, \mathrm{V})$ & $8.59(119)$ & $4(91)$ & $22(91)$ & & Fe II (13) & & & 0.219 (4) & & $0.22(151)$ \\
\hline CN Vel & 287.431 & 5.178 & $7.6(\mathrm{~V}, \mathrm{p})(14)$ & $10.2(84)$ & $400(84)$ & $>800$ (51) & & & & & $0.2204(89)$ & & \\
\hline CQ Vel & 272.333 & -4.895 & $12(\mathrm{j}, \mathrm{p})(14)$ & $8.9(15)$ & & $50(15)$ & & & & & & & \\
\hline LU Vul & 64.261 & 2.022 & $<11.5(\mathrm{p})(14)$ & $9.5(15)$ & $19(84)$ & $21(15)$ & & & & & & & \\
\hline V459 Vul & $\begin{array}{l}00.401 \\
58.214\end{array}$ & $\begin{array}{l}2.022 \\
-2.167\end{array}$ & 12.8 (V) (4) & $7.57(152)$ & 18 (153) & $30(153)$ & & & & $910(154)$ & & $\geqslant 2.2(6)$ & $0.86 \pm 0.12(153)$ \\
\hline
\end{tabular}

Notes: Notes same as in Table 1

References: (1) Strope et al. (2010), (2) Sheffer \& Krisciunas (1987), (3) Hachisu \& Kato (2014), (4) AAVSO database, (5) Tappert et al. (2014), (6) Özdönmez et al. (2016), (7) Hachisu \& Kato (2016a), (8) Ohshima et al. (1995), (9) Arkhipova et al. (2002), (10) Pagnotta \& Schaefer (2014), (11) Tappert et al. (2015), (12) Waagen (2012), (13) Walter et al. (2012), (14) Downes et al. (2001), (15) Shafter (1997), (16) Munari et al. (2014), (17) Srivastava et al. (2015), (18) Brosch \& Leibowitz (1981), (19) Hachisu \& Kato (2016b), (20) Helton et al. (2012), (21) Klare et al. (1980), (22) Slovak \& Vogt (1979), (23) Munari et al. (2008b), (24) Czart et al. (2006), (25) Balman et al. (2009), (26) Chochol et al. (2014), (27) Azaliah et al. (2015), (28) Hounsell et al. (20106), (29) Hounsell et al. (2010a), (30) Raj et al. (2013), (31) Yamaoka et al. (2009), (32) Ragan et al. (2009), (33) Selvelli \& Gilmozzi (2013), (34) Andrea et al. (1994), (35) Williams (2012), (36) Feast et al. (1991), (37) Szkody \& Ingram (1994), (38) Vanlandingham et al. (1996), (39) Shears \& Poyner (2007), (40) Wagner et al. (1991), (41) della Valle et al. (1994), (42) Wagner et al. (1994), (43) Ak et al. (2005), (44) Munari \& Dallaporta (2010), (45) in this study, (46) Munari et al. (2017a), (47) Srivastava et al. (2016), (48) Naik et al. (2010), (49) Siviero et al. (2005), (50) Ness et al. (2007), (51) Duerbeck (1987), (52) Orio et al. (2001 , (53) Burlak \& Henden (2008), (54) Cavagna et al. (1997), (55) Pojmanski et al. (2004), (56) Naito et al. (2005a), (57) Joshi et al. (2015), (58) Weight et al. (1994), (59) Williams (1994), (60) Chochol et al. (2005), (61) Miroshnichenko (1988), (62) Stringfellow et al. (1992), (63) della Valle et al. (1993), (64) Venturini et al. (2004), (65) Vanlandingham et al. (2001), (66) Livingston et al. (2001), (67) Fujii et al. (2002), (68) Duerbeck et al. (2002), (69) Kato et al. (2002a), (70) Wagner et al. (2003), (71) Vanlandingham et al. (2007), (72) Kato \& Fujii (2003), (73)

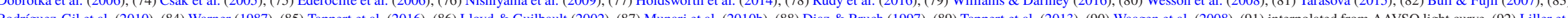

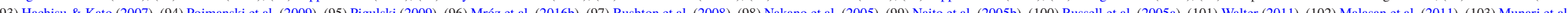
(2005), (104) Whitelock et al. (1984), (105) Cragg et al. (1983), (106) Diaz \& Steiner (1989), (107) Lyke et al. (2003), (108) Schwarz et al. (2011), (109) Senziani et al. (2008), (110) Puetter et al. (2004), (111) Pojmanski et al. (2006), (112) Russell et al. (2006), (113)

Ayani \& Murakami (2008), (114) Nakano et al. (2008a), (115) Russell et al. (2008), (116) Nakano et al. (2008b), (117) Helton et al. (2008b), (118) Rudy et al. (2008), (119) from AAVSO light curve, (120) Schmidtobreick et al. (2003), (121) Pereira et al. (2007), (122) Naik et al (201)

(2009). (123) Naito \& Tokimasa (2007), (124) Warner \& Woudt (2009), (125) Ness et al. (2008), (126) Read et al. (2008), (127) Fujii (1998), (128) Yamaoka et al. (2007b). (129) Naito et al. (2007), (130) Russell et al. (2007), (131) Nishivama et al. (2010), (132) Oksanen et al (2011),

(133) Henize \& McLaughlin (1951), (134) Richtler \& Liller (1986), (135) Kato et al. (2002b), (136) Munari et al. (2006b), (137) Fujii \& Yamaoka (2005), (138) Mazuk et al. (2005), (139) Rudy et al. (1999), (140) Yamaoka \& Fujii (2005), (141) Russell et al. (2005b), (142) Selvelli

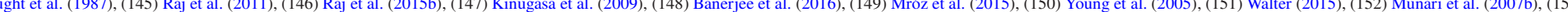
Poggiani (2010), (154) Nakano et al. (2007c) 
24 Özdönmez, A. et al.

This paper has been typeset from a $\mathrm{T}_{\mathrm{E}} \mathrm{X} / \mathrm{L} \mathrm{AT} \mathrm{E} \mathrm{X}$ file prepared by the author. 